\title{
COSMOS MORPHOLOGICAL CLASSIFICATION WITH THE ZURICH ESTIMATOR OF STRUCTURAL TYPES (ZEST) AND THE EVOLUTION SINCE $z=1$ OF THE LUMINOSITY FUNCTION OF EARLY, DISK, AND IRREGULAR GALAXIES ${ }^{1}$
}

\author{
C. Scarlata, ${ }^{2}$ C. M. Carollo, ${ }^{2}$ S. Lilly,${ }^{2}$ M. T. Sargent,${ }^{2}$ R. Feldmann, ${ }^{2}$ P. Kampczyk ${ }^{2}$ C. Porciani, ${ }^{2}$ \\ A. Koekemoer, ${ }^{3}$ N. Scoville, ${ }^{4}$ J.-P. Kneib, ${ }^{5}$ A. Leauthaud, ${ }^{5}$ R. Massey, ${ }^{4}$ J. Rhodes, ${ }^{4,6}$ L. Tasca, \\ P. Capak, ${ }^{4}$ C. Maier ${ }^{2}$ H. J. McCracken, ${ }^{7}$ B. Mobasher, ${ }^{3}$ A. Renzini, ${ }^{8}$ Y. Taniguchi, ${ }^{9}$ \\ D. Thompson, ${ }^{4,10}$ K. Sheth, ${ }^{4,11}$ M. Ajiki, ${ }^{12}$ H. Aussel, ${ }^{13,14}$ T. Murayama, ${ }^{12}$ \\ D. B. SANDERs, ${ }^{13}$ S. SASAKi, ${ }^{12,15}$ Y. ShIOYA, ${ }^{15}$ AND M. TAKAhashi ${ }^{12,15}$ \\ Received 2006 April 25; accepted 2006 November 5
}

\begin{abstract}
Motivated by the desire to reliably and automatically classify structure of thousands of COSMOS galaxies, we present ZEST, the Zurich Estimator of Structural Types. To classify galaxy structure, ZEST uses (1) five nonparametric diagnostics: asymmetry, concentration, Gini coefficient, second-order moment of the brightest $20 \%$ of galaxy pixels, and ellipticity; and (2) the exponent $n$ of single-Sérsic fits to the two-dimensional surface brightness distributions. To fully exploit the wealth of information while reducing the redundancy present in these diagnostics, ZEST performs a principal component (PC) analysis. We use a sample of $\sim 56,000 I_{\mathrm{AB}} \leq 24$ COSMOS galaxies to show that the first three PCs fully describe the key aspects of the galaxy structure, i.e., to calibrate a three-dimensional classification grid of axes $\mathrm{PC}_{1}, \mathrm{PC}_{2}$, and $\mathrm{PC}_{3}$. We demonstrate the robustness of the ZEST grid on the $z=0$ sample of Frei et al. The ZEST classification breaks most of the degeneracy between different galaxy populations that affects morphological classifications based on only some of the diagnostics included in ZEST. As a first application, we present the evolution since $z \sim 1$ of the luminosity functions (LFs) of COSMOS galaxies of early, disk, and irregular galaxies and, for disk galaxies, of different bulge-to-disk ratios. Overall, we find that the LF up to a redshift $z=1$ is consistent with a pure luminosity evolution (of about 0.95 mag at $z \sim 0.7$ ). We highlight, however, two trends that are in general agreement with a downsizing scenario for galaxy formation, i.e., (1) a deficit of a factor of about 2 at $z \sim 0.7$ of $M_{B}>-20.5$ structurally classified earlytype galaxies and (2) an excess of a factor of about 3, at a similar redshift, of irregular galaxies.
\end{abstract}

Subject headings: cosmology: observations — dark matter — galaxies: evolution — galaxies: formation — large-scale structure of universe - surveys

Online material: color figures

\section{INTRODUCTION}

Galaxy morphologies are strongly correlated with the star formation histories of galaxies, are a proxy for their dynamical structure, and are thus a key diagnostic of galaxy evolution. As state-of-the-art surveys of faint galaxies at (low and) high redshifts such as COSMOS now include millions of galaxies, it becomes essential to have robust tools for automatic morphological and structural classifications.

Motivated by the need to automatically derive a quantitative description for the morphology of the large number of COSMOS

\footnotetext{
1 Based on observations with the NASA/ESA Hubble Space Telescope, obtained at the Space Telescope Science Institute, which is operated by the Association of Universities for Research in Astronomy (AURA), Inc., under NASA contract NAS 5-26555; also based on data collected at the Subaru Telescope, which is operated by the National Astronomical Observatory of Japan; Kitt Peak National Observatory, Cerro Tololo Inter-American Observatory, and the National Optical Astronomy Observatory, which are operated by AURA, Inc., under cooperative agreement with the National Science Foundation; and the Canada-France-Hawaii Telescope operated by the National Research Council of Canada, the Centre National de la Recherche Scientifique de France, and the University of Hawaii.

2 Department of Physics, Swiss Federal Institute of Technology (ETH-Zurich), CH-8093 Zurich, Switzerland.

3 Space Telescope Science Institute, 3700 San Martin Drive, Baltimore, MD 21218.

4 California Institute of Technology, MC 105-24, 1200 East California Boulevard, Pasadena, CA 91125.

5 Laboratoire d'Astrophysique de Marseille, BP 8, Traverse du Siphon, 13376 Marseille Cedex 12, France.
}

galaxies, we present the Zurich Estimator of Structural Types (ZEST), a powerful classification scheme that combines:

1. A principal component analysis (PCA) of five nonparametric diagnostics of galaxy structure, i.e., asymmetry $A$, concentration $C$, Gini coefficient $\mathrm{G}$, second-order moment of the brightest $20 \%$ of galaxy pixels $M_{20}$ (e.g., Abraham et al. 2003; Lotz et al. 2004), and the ellipticity $\epsilon$. The PCA reduces the redundancy of information that is present in these five diagnostics, without substantial loss of information about the galaxy structure.

\footnotetext{
6 Jet Propulsion Laboratory, Pasadena, CA 91109.

7 Institut d'Astrophysique de Paris, UMR 7095, 98 bis Boulevard Arago, 75014 Paris, France.

8 Dipartimento di Astronomia, Universitá di Padova, vicolo dell'Osservatorio 2, I-35122 Padua, Italy.

9 Subaru Telescope, National Astronomical Observatory of Japan, 650 North A'ohoku Place, Hilo, HI 96720.

${ }^{10}$ Large Binocular Telescope Observatory, University of Arizona, 933 North Cherry Avenue, Tucson, AZ 85721.

11 Spitzer Science Center, California Institute of Technology, Pasadena, CA 91125.

12 Astronomical Institute, Graduate School of Science, Tohoku University, Aramaki, Aoba, Sendai 980-8578, Japan.

13 Institute for Astronomy, 2680 Woodlawn Drive, University of Hawaii, Honolulu, HI, 96822.

14 Service d'Astrophysique, CEA/Saclay, 91191 Gif-sur-Yvette, France.

15 Physics Department, Graduate School of Science and Engineering, Ehime University, Matsuyama, Japan.
} 
2. A parametric description of the galaxy light, i.e., the exponent $n$ of a single-Sérsic fit to its surface brightness distribution (Sargent et al. 2007).

Specifically, ZEST classifies galaxies on the basis of their location in the three-dimensional space defined by the main three eigenvectors $\mathrm{PC}_{1}, \mathrm{PC}_{2}$, and $\mathrm{PC}_{3}$ that contain most of the variance of the original nonparametric quantities, while the $n$ Sérsic indices are used to refine the classification of disk galaxies by splitting these in four separate bins of a bulgeness parameter (that is correlated with the bulge-to-disk ratio $[\mathrm{B} / \mathrm{D}])$.

We calibrate the ZEST grid with a sample of $\sim 56,000 I_{\mathrm{AB}} \leq$ 24 COSMOS galaxies selected from a catalog covering an area of about $40 \%$ of the entire $2 \mathrm{deg}^{2}$ COSMOS field, produced from the Cycle 12 Hubble Space Telescope (HST) Advanced Camera for Surveys (ACS) F814W images (Scoville et al. 2007a; Leauthaud et al. 2007). The ZEST calibration is also tested on the $z=0$ sample of Frei et al. (1996). The ZEST grid assigns, to each galaxy:

1. A morphological type $(=1,2$, or 3 for early-type, disk, and irregular galaxies, respectively);

2. A bulgeness parameter for the type 2 disk galaxies, which coarsely correlates with $\mathrm{B} / \mathrm{D}$; the disk galaxies are split in four bins, from pure disk galaxies $(T=2.3)$ to bulge-dominated disks $(T=2.0)$;

3. An elongation parameter (four bins from 0 for face-on to 3 for edge-on galaxies);

4. An irregularity (for type 1) and a clumpiness parameter (for type 1 and 2), which indicate whether the galaxy light distribution is regular/smooth or distorted/clumpy, respectively.

Although the size of the galaxy does not enter directly in the ZEST classification, a measurement of the Petrosian radius (Petrosian 1976) is produced as a by-product of the ZEST classification procedure and is thus also available for all the ZESTclassified galaxies. The calibrated ZEST grid in the $\mathrm{PC}_{1}-\mathrm{PC}_{2}-\mathrm{PC}_{3}$ space can be used to automatically classify galaxies according to their structural properties.

While different combinations of some of the basic diagnostics that we use to construct the ZEST grid have been extensively used in the literature to classify galaxy types, these combinations are effectively "projections" on lower dimensionality planes of the ZEST $\mathrm{PC}_{1}-\mathrm{PC}_{2}-\mathrm{PC}_{3}$ space. These projections mix very different galaxy populations (e.g., elliptical galaxies with regular edge-on disks in the $M_{20}-\mathrm{G}$ and $C-M_{20}$ planes, etc.) and thus lead to heavy contamination of galaxy samples and erroneous statistical assessments concerning the evolution of specific galaxy populations. The three-dimensional $\mathrm{PC}_{1}-\mathrm{PC}_{2}-\mathrm{PC}_{3}$ space breaks the degeneracy between these different galaxy populations.

As a first application of the ZEST structural classification of COSMOS galaxies, we present the redshift evolution since $z=1$ of the luminosity functions (LFs) of galaxies with different structural types (including different "bulgeness" for the disk galaxies). In order to anchor the COSMOS measurements to the local universe, we use the sample of 1813 Sloan Digital Sky Survey (SDSS; York et al. 2000) galaxies constructed and discussed by Kampczyk et al. (2007). A detailed discussion of the LFs of more elaborated selections of galaxy samples that are structurally classified with ZEST is presented elsewhere (see, e.g., Scarlata et al. [2007] for an extensive discussion of the evolution of the LF of elliptical galaxy progenitors).

This paper is structured as follows. In $\S 2$ we describe the methodology at the basis of the ZEST classification: in particular, $\S 2.1$ describes the nonparametric quantification of galaxy structure; $\S 2.2$ the PC analysis in the $A, C, \mathrm{G}, M_{20}$, and $\epsilon$ space; $\S 2.3$ presents the calibration of the ZEST morphological grid, as well as the use of the $n$ Sérsic index values to establish the B/D of disk galaxies ( $\S 2.3 .2$ ). The performance and reliability of ZEST are discussed in $\S 3$, where we present the test performed on a $z=0$ galaxy sample $(\S 3.1)$ and a discussion of the error bars in the ZEST structural classification ( $\S 3.2)$. In $\S 4$ we show the advantage of the ZEST classification scheme over other approaches. These comparisons show that the ZEST classification is substantially more powerful, relative to other schemes that are based on only a few of the basic diagnostics that are contained in the ZEST grid, in separating galaxies with different structural properties. In $\S 5$ we present the LFs of the ZEST-classified COSMOS galaxies, the corrections applied to the LFs, and the sources of error. The paper is briefly summarized in $\S 6$.

Throughout the paper we use the cosmology that has been adopted throughout this volume, i.e., $\Omega_{m}=0.25, \Omega_{m}+\Omega_{\Lambda}=1$, and $H_{0}=70 \mathrm{~km} \mathrm{~s}^{-1} \mathrm{Mpc}^{-1}$. All magnitudes are expressed in the AB system (Oke 1974).

\section{METHODOLOGY}

ZEST is based on a combination of nonparametric and parametric quantification of galaxy structure, and a PCA to reduce the number of variables. We describe below the steps that we followed to construct the ZEST classification grid.

\subsection{Nonparametric Quantification of Structure}

The ZEST PCA uses, as basic quantities, four widely adopted nonparametric measurements of the light distribution in galaxies (e.g., Abraham et al. 2003; Lotz et al. 2004), plus a measurement of the ellipticity of the galaxy light distribution. Specifically, ZEST uses

1. The concentration $C\left[=5 \log \left(r_{80} / r_{20}\right)\right.$; with $r_{80}$ and $r_{20}$ the radii including $80 \%$ and $20 \%$ of the total galaxy light, respectively], which quantifies the central density of the galaxy light distribution;

2. The asymmetry $A$, which quantifies the degree of rotational symmetry of the light distribution. The quantity $A$ is measured by calculating the normalized difference between the galaxy image and the image rotated by $180^{\circ}$. A correction for background noise is also applied (as in Conselice et al. 2000; Lotz et al. 2004), i.e.,

$$
A=\frac{\sum_{x, y}\left|I_{(x, y)}-I_{180(x, y)}\right|}{2 \sum\left|I_{(x, y)}\right|}-B_{180}
$$

where $I$ is the galaxy flux in pixel $(x, y), I_{180}$ is the image rotated by $180^{\circ}$ about the galaxy's central pixel, and $B_{180}$ is the average asymmetry of the background;

3. The Gini coefficient $\mathrm{G}$, which describes how uniformly the flux is distributed among galaxy pixels. The Gini statistic assumes values from 0 (if the galaxy light is homogeneously distributed among galaxy pixels) up to 1 (if all the light is concentrated in 1 pixel, regardless of its position in the galaxy). Specifically, after ordering the pixels by increasing flux value, $\mathrm{G}$ is given by

$$
\mathrm{G}=\frac{1}{\bar{X} n(n-1)} \sum_{i}^{n}(2 i-n-1) X_{i},
$$

where $n$ is the number of pixels assigned to a galaxy and $\bar{X}$ is the mean pixel value (Glasser 1962); 


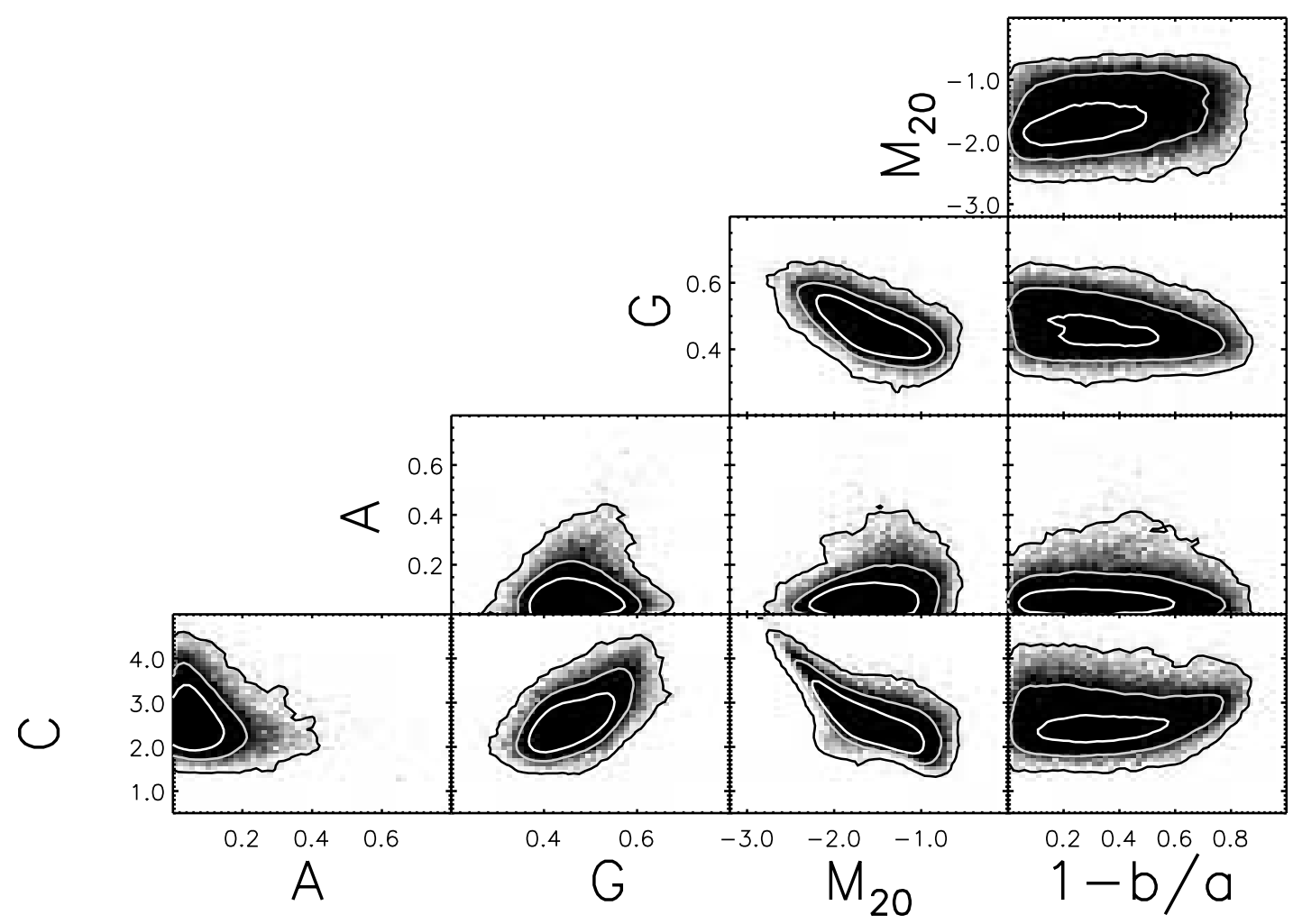

FIG. 1.- Relations between the nonparametric diagnostics $\left(M_{20}, \mathrm{G}, A, C\right.$, and $\left.\epsilon=1-b / a\right)$. Contours enclose $\sim 30 \%$ (white contour), $80 \%$ (gray contour), and $98 \%$ (black contour) of the galaxies. The main correlations among some of the parameters, such as $M_{20}, C$, and $\mathrm{G}$, are clearly visible in these diagrams.

4. $M_{20}\left(=\log \sum M_{i} / M_{\text {tot }}\right.$, with $\sum f_{i}<20 \%$ and $M_{\text {tot }}$ the total second-order moment), i.e., the second-order moment of the brightest $20 \%$ of the galaxy flux. For centrally concentrated objects, $M_{20}$ correlates with the concentration $C$; however, $M_{20}$ is also sensitive to bright off-centered knots of light;

5. The ellipticity $\epsilon=1-b / a$ of the light distribution, as measured by SExtractor (ver. 2.4.3; Bertin \& Arnouts 1996). SExtractor computes the semimajor axis $a$ and semiminor axis $b$ from the second-order moments of the galaxy light; specifically, $a$ and $b$ are the maximum and minimum spatial variance (rms) of the object, along the direction $\theta$ where the variance is maximized:

$$
\begin{aligned}
& a^{2}=\frac{\bar{x}^{2}+\bar{y}^{2}}{2}+\sqrt{\frac{\left(\bar{x}^{2}-\bar{y}^{2}\right)^{2}}{4}+\bar{x} y^{2},} \\
& b^{2}=\frac{\bar{x}^{2}+\bar{y}^{2}}{2}-\sqrt{\frac{\left(\bar{x}^{2}-\bar{y}^{2}\right)^{2}}{4}+\bar{x} y^{2}},
\end{aligned}
$$

where the second-order moments $\bar{x} 2, \bar{y} 2$, and $\bar{x} y$ are given by

$$
\begin{aligned}
& \bar{x}^{2}=\frac{\sum_{i} I_{i} x_{i}^{2}}{\sum I_{i}}-\bar{x}^{2}, \\
& \bar{y}^{2}=\frac{\sum_{i} I_{i} y_{i}^{2}}{\sum I_{i}}-\bar{y}^{2}, \\
& \bar{x} y=\frac{\sum_{i} I_{i} x_{i} y_{i}}{\sum I_{i}}-\bar{x} \bar{y} .
\end{aligned}
$$

The values of $a$ and $b$ are thus representative of the galaxy ellipticity at large radii and are rather insensitive to details in the internal structure (e.g., bars in disk galaxies, knots of star formation, etc.).

\subsection{Principal Component Analysis}

The quantities above provide complementary, but also redundant, information on galaxy structure. We therefore performed a PCA using the measurements of $A, C, M_{20}$, G, and ellipticity $\epsilon$ as basic variables.

The PCA is a classical statistical method for multivariate analysis, which reduces the dimensionality of a data set without a

\begin{tabular}{|c|c|c|c|c|c|}
\hline $\begin{array}{l}\text { Variable } \\
\text { (1) }\end{array}$ & $\begin{array}{l}\mathrm{PC}_{1} \\
(2)\end{array}$ & $\begin{array}{l}\mathrm{PC}_{2} \\
\text { (3) }\end{array}$ & $\begin{array}{l}\mathrm{PC}_{3} \\
\text { (4) }\end{array}$ & $\begin{array}{l}\mathrm{PC}_{4} \\
(5)\end{array}$ & $\begin{array}{l}\mathrm{PC}_{5} \\
(6)\end{array}$ \\
\hline Eigenvalue............ & 2.46 & 1.19 & 0.92 & 0.25 & 0.17 \\
\hline Proportion...................................... & 0.49 & 0.24 & 0.18 & 0.05 & 0.03 \\
\hline 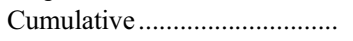 & 0.49 & 0.73 & 0.92 & 0.97 & 1.00 \\
\hline Concentration $\left(=x_{1}\right) \ldots \ldots \ldots \ldots \ldots . . . . . .$. & -0.54 & 0.35 & 0.18 & -0.34 & -0.66 \\
\hline$M_{20}\left(=x_{2}\right) \ldots \ldots \ldots \ldots \ldots \ldots$ & 0.60 & -0.04 & 0.03 & 0.39 & -0.70 \\
\hline Gini $\left(=x_{3}\right) \ldots \ldots \ldots \ldots \ldots \ldots$ & -0.56 & -0.20 & 0.14 & 0.79 & -0.02 \\
\hline 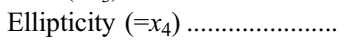 & 0.20 & 0.57 & 0.74 & 0.16 & 0.26 \\
\hline Asymmetry $\left(=x_{5}\right) \ldots \ldots \ldots \ldots \ldots \ldots$ & 0.02 & -0.71 & 0.64 & -0.29 & -0.08 \\
\hline
\end{tabular}
significant loss of information. This is done by transforming the observed variables into a new set of orthogonal variables, the

TABLE 1

Results of the ZEST PC ANALYsis

Notes.-Columns (2)-(6) refer to the five PCs in ZEST. The first row gives the eigenvalue (i.e., variance) of the data along the direction of the corresponding PC. The second and third rows show the fraction of variance and the cumulative fraction of each PC, respectively. The last five rows list the weights assigned to each input variable, in the linear combination that gives the direction of the PC (i.e., $\mathrm{PC}_{i}=\alpha x_{1}+\beta x_{2}+\gamma x_{3}+\delta x_{4}+\phi x_{5}$, with the coefficients $\alpha, \ldots, \phi$ given by the numbers listed, per each PC, in the last five rows). 


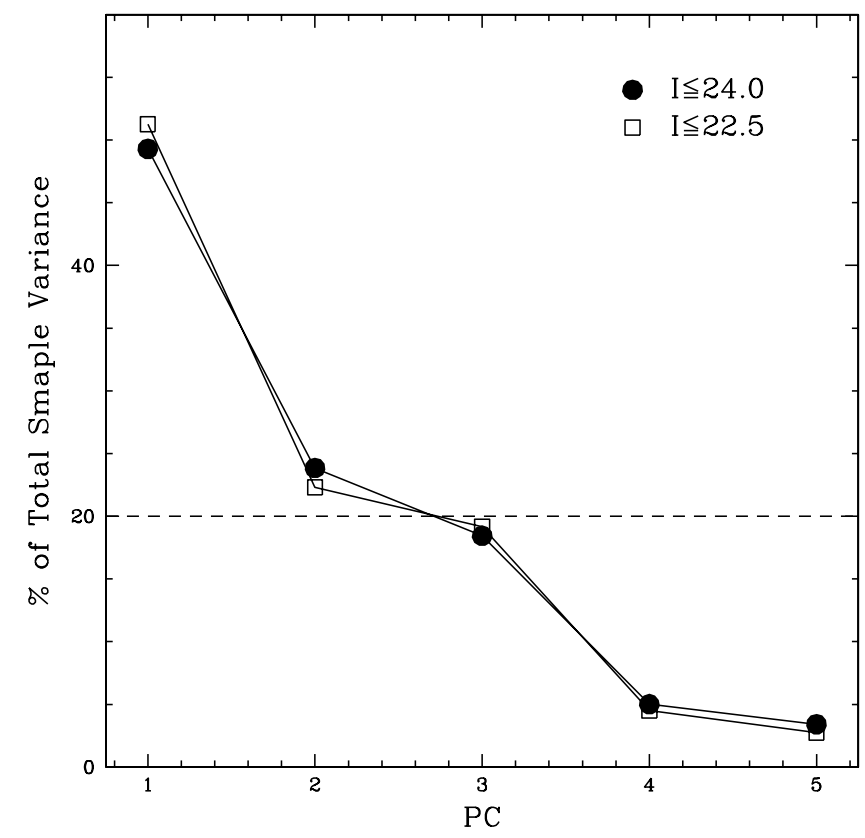

FIG. 2.- Fraction of the total variance explained by each PC as a function of the corresponding PCs for all galaxies down to $I=24$ in the ACS catalog ( filled circles). Squares refer to the same analysis performed only on those objects with magnitude brighter than $I=22.5$. The horizontal line indicates $20 \%$ of the total variance, i.e., the value for the five eigenvalues for a sample of $100 \%$ uncorrelated variables.

principal components " $\mathrm{PC}_{i}$ ", (with $i=1, \ldots, n$, and $n$ the number of basic parameters, i.e., variables). The $\mathrm{PC}_{i}$ are ordered so that the first few of them retain most of the variance present in the original data set. The PCs are a linear combination of the original variables and define a new coordinate system obtained by rigid rotation of the original space. In the new system, the axes represent the directions of maximum variability in the original $n$-dimensional distribution of points.

In detail, the data set is described by an $n \times m$ data matrix ( $n=5$ in the current version of ZEST), and $m$ is the number of galaxies with measured basic parameters. All variables are standardized before performing the analysis by subtracting their median value (indicated with the subscript $M$ in the following expressions) and normalizing them with their standard deviation. Therefore, the five variables considered in the ZEST PCA are defined as $x_{1}=\left(C-C_{M}\right) / \sigma_{C}, x_{2}=\left(M_{20}-M_{20, M}\right) / \sigma_{M_{20}}, x_{3}=$ $\left(\mathrm{G}-\mathrm{G}_{M}\right) / \sigma_{\mathrm{G}}, x_{4}=\left(\epsilon-\epsilon_{M}\right) / \sigma_{\epsilon}$, and $x_{5}=\left(A-A_{M}\right) / \sigma_{A}$.
The directions of the PCs are derived by calculating the eigenvectors of the $n \times n$ covariance matrix of the $x_{j}$ variables $\left[S_{i j}=\right.$ $\left.\left\langle\left(x_{i}-\left\langle x_{i}\right\rangle\right)\left(x_{j}-\left\langle x_{j}\right\rangle\right)\right\rangle\right]$. The matrix $S \geq 0$ is real and symmetric. Thus, it admits real, positive eigenvalues $\lambda_{i}$. By sorting the eigenvectors in order of decreasing values of the eigenvalues, an ordered orthogonal basis is obtained, with eigenvectors aligned along directions of decreasing variance $\left(\lambda_{i} / \sum_{j} \lambda_{j}\right)$ in the data. The first few PCs that account for most of the power, i.e., most of the total variance, $\sum_{j} \lambda_{j}$, in the data set, are then used to replace the original $n$ variables without any significant loss of information.

\subsection{ZEST Calibration with $56,000 I_{\mathrm{AB}} \leq 24$ COSMOS Galaxies}

We calibrate the ZEST classification grid on a sample of $\sim 56,000, I_{\mathrm{AB}} \leq 24$, COSMOS galaxies detected in the $260 \mathrm{ACS}$ F814W images acquired during the HST Cycle 12 observing period (Scoville et al. 2007a). The total area covered by this fraction of COSMOS is $0.74 \mathrm{deg}^{2}$. Details on the COSMOS sample are given in Appendix A.1.

For each COSMOS galaxy, we measured the basic nonparametric quantities described in $\S 2.1$ by computing them on the galaxy pixels (defined using Petrosian apertures, see Appendix A.2). Figure 1 shows the behavior of each basic nonparametric diagnostic as a function of the others. The contours in each panel enclose $30 \%, 80 \%$, and $98 \%$ of the COSMOS galaxies in our sample. Global correlations are known to exist between various nonparametric coefficients. For example, relatively tight correlations exist between $\mathrm{G}, M_{20}$, and $C$, with objects with high $C$ tending to have low $M_{20}$ and high G. Any value of $C$ is observed for small values of $A$, while high values of $A$ are preferentially observed in low- $C$ galaxies. These trends have already been noted in the literature and indeed highlight the redundancy of information present in these diagnostics. As expected, the ellipticity $\epsilon$ does not correlate with any of the other parameters, except for a mild positive correlation with the concentration for $\epsilon>0.6$. This is a geometric effect, since edge-on galaxies preferentially have high $C$-values.

The results of the PCA on the normalized COSMOS data set are presented in Table 1. In particular, columns (2)-(6) refer to the five PCs derived in the analysis. The first row gives the eigenvalue (i.e., variance) of the data along the direction of the corresponding PC. The second row shows the fraction of the variance that is explained by each of the PCs, i.e., the fraction of the "power" that is contained in each PC; the third row lists the cumulative fraction of the variance. In the last five rows of the table, each column lists the weights assigned to each input variable

TABLE 2

The ZEST Classification Scheme

\begin{tabular}{|c|c|c|c|}
\hline Parameter & $\begin{array}{c}\text { Type } 1 \\
\text { Early Types } \\
\text { (No Visible Disk) }\end{array}$ & $\begin{array}{c}\text { Type } 2 \\
\text { Disk Galaxies }\end{array}$ & $\begin{array}{c}\text { Type } 3 \\
\text { Irregular Galaxies }\end{array}$ \\
\hline 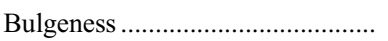 & & From 0 (massive bulge) to 3 (bulgeless disk) & . \\
\hline Elongation & From 0 (face on) to 3 (edge on) & From 0 (face on) to 3 (edge on) & $\ldots$ \\
\hline 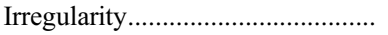 & From 0 (regular) to 2 (highly irregular) & $\ldots$ & ... \\
\hline 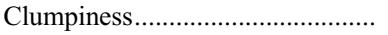 & From 0 (smooth) to 3 (very clumpy) & From 0 (smooth) to 3 (very clumpy) & $\ldots$ \\
\hline Size & $R_{\mathrm{P}}$ & $R_{\mathrm{P}}$ & $R_{\mathrm{P}}$ \\
\hline
\end{tabular}

Notes. - Summary of the ZEST classification scheme. Type 1 (early-type galaxies) are spheroids with no visible disk (including face-on S0 galaxies, for which the identification of the disk component is difficult). Type 2 are disk galaxies, and type 3 are irregular galaxies. A clumpiness parameter is assigned to each ZEST unit cube classified as type 1 or 2, Early-type and disk galaxies are assigned an elongation parameter in four steps from 0 (face on) to 3 (edge on), and an irregularity parameter from 0 for regular to 2 for highly irregular galaxies. Type 2 disk galaxies are split in four bins of bulgeness parameter, namely, 2.0, 2.1, 2.2, and 2.3 from bulge-dominated to bulgeless disks. Relatively inclined S0 galaxies occupy cubes classified as $T=2.0$. The last row indicates that a measure of galaxy size (the Petrosian radius, Petrosian 1976) is available for all ZEST-classified galaxies, as a by-product of our analysis. 
TABLE 3

COSMOS ZEST CLASSIFICATION GRID

\begin{tabular}{|c|c|c|c|c|c|c|c|c|c|c|}
\hline \multirow[b]{2}{*}{$\mathrm{PC}_{2}$} & \multicolumn{10}{|c|}{$\mathrm{PC}_{1}$} \\
\hline & -6 & -5 & -4 & -3 & -2 & -1 & 0 & 1 & 2 & 3 \\
\hline \multicolumn{11}{|c|}{$\mathrm{PC}_{3}=-2$} \\
\hline$-4 \ldots \ldots \ldots$ & $\ldots$ & $\ldots$ & $\ldots$ & $\ldots$ & $\ldots$ & $\ldots$ & $\ldots$ & $\ldots$ & $\cdots$ & \\
\hline$-3 \ldots \ldots \ldots$ & $\ldots$ & $\ldots$ & $\ldots$ & $\ldots$ & $\ldots$ & $\ldots$ & $\ldots$ & $\ldots$ & $\ldots$ & \\
\hline$-2 \ldots \ldots \ldots$ & $\ldots$ & $\ldots$ & $\ldots$ & $\ldots$ & $\ldots$ & $\ldots$ & $\ldots$ & $\ldots$ & $\ldots$ & $\ldots$ \\
\hline$-1 \ldots \ldots \ldots$ & $\ldots$ & $\ldots$ & $\ldots$ & $\ldots$ & $\ldots$ & 2.2 & 2.2 & 2.3 & 2.3 & 2.3 \\
\hline $0 \ldots \ldots \ldots \ldots$ & $\ldots$ & $\ldots$ & $\ldots$ & $\ldots$ & 2.1 & 2.2 & 2.2 & 2.3 & 2.3 & 2.3 \\
\hline $1 \ldots \ldots \ldots . . . .$. & $\ldots$ & $\ldots$ & $\ldots$ & $\ldots$ & 2.2 & 2.2 & 2.2 & 2.2 & 2.2 & 2.2 \\
\hline $2 \ldots \ldots \ldots \ldots . .$. & $\cdots$ & $\ldots$ & $\ldots$ & $\cdots$ & $\ldots$ & $\ldots$ & $\ldots$ & $\cdots$ & $\cdots$ & $\ldots$ \\
\hline $3 \ldots \ldots \ldots \ldots$ & $\ldots$ & $\cdots$ & $\ldots$ & $\ldots$ & $\ldots$ & $\ldots$ & $\ldots$ & $\ldots$ & $\ldots$ & $\ldots$ \\
\hline
\end{tabular}

\begin{tabular}{lllllllllll}
\hline$-4 \ldots \ldots \ldots$. & $\ldots$ & $\ldots$ & $\ldots$ & $\ldots$ & $\ldots$ & $\ldots$ & $\ldots$ & $\ldots$ & $\ldots$ & $\ldots$ \\
$-3 \ldots \ldots \ldots$. & $\ldots$ & $\ldots$ & $\ldots$ & $\ldots$ & $\ldots$ & $\ldots$ & $\ldots$ & $\ldots$ & $\ldots$ & $\ldots$ \\
$-2 \ldots \ldots \ldots$. & $\ldots$ & $\ldots$ & $\ldots$ & $\ldots$ & 1 & 2.2 & 2.2 & 2.3 & 3 & 3 \\
$-1 \ldots \ldots \ldots$. & $\ldots$ & $\ldots$ & 1 & 1 & 2.1 & 2.1 & 2.2 & 2.3 & 2.3 & 2.3 \\
$0 \ldots \ldots \ldots \ldots$. & $\ldots$ & $\ldots$ & 1 & 2.0 & 2.1 & 2.1 & 2.2 & 2.3 & 2.3 & 2.3 \\
$1 \ldots \ldots \ldots \ldots$ & $\ldots$ & $\ldots$ & 2.0 & 2.0 & 2.1 & 2.1 & 2.2 & 2.3 & 2.3 & 2.3 \\
$2 \ldots \ldots \ldots \ldots$. & $\ldots$ & $\ldots$ & $\ldots$ & $\ldots$ & $\ldots$ & $\ldots$ & $\ldots$ & $\ldots$ & $\ldots$ & $\ldots$ \\
$3 \ldots \ldots \ldots \ldots$. & $\ldots$ & $\ldots$ & $\ldots$ & $\ldots$ & $\ldots$ & $\ldots$ & $\ldots$ & $\ldots$ & $\ldots$ & $\ldots$
\end{tabular}

\begin{tabular}{llllllllllll}
\hline \multicolumn{10}{c}{$\mathrm{PC}_{3}=0$} \\
\hline$-4 \ldots \ldots \ldots$ & $\ldots$ & $\ldots$ & $\ldots$ & $\ldots$ & $\ldots$ & $\ldots$ & $\ldots$ & $\ldots$ & $\ldots$ & $\ldots$ \\
$-3 \ldots \ldots \ldots$. & $\ldots$ & $\ldots$ & $\ldots$ & $\ldots$ & 2.1 & 3 & 3 & 3 & 3 & 3 \\
$-2 \ldots \ldots \ldots$ & $\ldots$ & $\ldots$ & 1 & 1 & 2.0 & 2.1 & 3 & 3 & 3 & 3 \\
$-1 \ldots \ldots \ldots$. & $\ldots$ & 1 & 1 & 1 & 2.1 & 2.1 & 2.2 & 2.3 & 2.3 & 3 \\
$0 \ldots \ldots \ldots \ldots$ & 1 & 1 & 1 & 2.0 & 2.1 & 2.1 & 2.2 & 2.3 & 2.3 & 2.3 \\
$1 \ldots \ldots \ldots \ldots$ & $\ldots$ & 1 & 1 & 2.0 & 2.1 & 2.1 & 2.2 & 2.3 & 2.3 & 2.3 \\
$2 \ldots \ldots \ldots \ldots$. & $\ldots$ & $\ldots$ & 2.0 & 2.1 & 2.1 & 2.1 & 2.2 & 2.2 & 2.3 & 2.3 \\
$3 \ldots \ldots \ldots \ldots$. & $\ldots$ & $\ldots$ & $\ldots$ & $\ldots$ & $\ldots$ & $\ldots$ & $\ldots$ & $\ldots$ & $\ldots$ & $\ldots$ \\
\hline
\end{tabular}

$$
\mathrm{PC}_{3}=1
$$

\begin{tabular}{lllllllllll}
\hline$-4 \ldots \ldots \ldots$. & $\ldots$ & $\ldots$ & $\ldots$ & $\ldots$ & $\ldots$ & $\ldots$ & $\ldots$ & $\ldots$ & $\ldots$ & $\ldots$ \\
$-3 \ldots \ldots \ldots$. & $\ldots$ & $\ldots$ & $\ldots$ & $\ldots$ & $\ldots$ & 3 & 3 & 3 & 3 & 3 \\
$-2 \ldots \ldots \ldots$. & $\ldots$ & $\ldots$ & 1 & 2.0 & 2.0 & 3 & 3 & 3 & 3 & 3 \\
$-1 \ldots \ldots \ldots$. & $\ldots$ & 1 & 1 & 2.0 & 2.0 & 2.1 & 2.2 & 3 & 3 & 3 \\
$0 \ldots \ldots \ldots \ldots$. & 1 & 1 & 2.0 & 2.0 & 2.1 & 2.1 & 2.2 & 2.3 & 2.3 & 3 \\
$1 \ldots \ldots \ldots \ldots$. & 1 & 1 & 2.0 & 2.0 & 2.1 & 2.2 & 2.2 & 2.3 & 2.3 & 2.3 \\
$2 \ldots \ldots \ldots \ldots$. & $\ldots$ & $\ldots$ & 2.0 & 2.1 & 2.1 & 2.2 & 2.2 & 2.3 & 2.3 & 2.3 \\
$3 \ldots \ldots \ldots \ldots$. & $\ldots$ & $\ldots$ & $\ldots$ & $\ldots$ & $\ldots$ & 2.2 & 2.2 & 2.2 & 2.3 & $\ldots$ \\
\hline
\end{tabular}

$$
\mathrm{PC}_{3}=2
$$

\begin{tabular}{lllllllllll}
\hline$-4 \ldots \ldots \ldots$ & $\ldots$ & $\ldots$ & $\ldots$ & $\ldots$ & $\ldots$ & 3 & 3 & 3 & 3 & 3 \\
$-3 \ldots \ldots \ldots$. & $\ldots$ & $\ldots$ & $\ldots$ & $\ldots$ & 1 & 3 & 3 & 3 & 3 & 3 \\
$-2 \ldots \ldots \ldots$. & $\ldots$ & $\ldots$ & $\ldots$ & 1 & 1 & 3 & 3 & 3 & 3 & 3 \\
$-1 \ldots \ldots \ldots$. & $\ldots$ & $\ldots$ & 1 & 2.0 & 2.0 & 3 & 3 & 3 & 3 & 3 \\
$0 \ldots \ldots \ldots \ldots$ & $\ldots$ & 2.0 & 2.0 & 2.0 & 2.1 & 2.1 & 2.2 & 2.3 & 3 & 3 \\
$1 \ldots \ldots \ldots \ldots$. & 2.0 & 2.0 & 2.0 & 2.0 & 2.1 & 2.2 & 2.2 & 2.3 & 2.3 & 2.3 \\
$2 \ldots \ldots \ldots \ldots$. & $\ldots$ & 2.0 & 2.0 & 2.0 & 2.1 & 2.2 & 2.3 & 2.3 & 2.3 & 2.3 \\
$3 \ldots \ldots \ldots \ldots$. & $\ldots$ & $\ldots$ & $\ldots$ & 2.1 & 2.1 & 2.2 & 2.3 & 2.3 & 2.3 & $\ldots$ \\
\hline
\end{tabular}

\begin{tabular}{|c|c|c|c|c|c|c|c|c|c|c|}
\hline \multirow[b]{2}{*}{$\mathrm{PC}_{2}$} & \multicolumn{10}{|c|}{$\mathrm{PC}_{1}$} \\
\hline & -6 & -5 & -4 & -3 & -2 & -1 & 0 & 1 & 2 & 3 \\
\hline \multicolumn{11}{|c|}{$\mathrm{PC}_{3}=3$} \\
\hline$-4 \ldots \ldots \ldots \ldots \ldots$ & $\ldots$ & $\ldots$ & $\ldots$ & $\ldots$ & $\ldots$ & $\ldots$ & 3 & 3 & 3 & .. \\
\hline$-3 \ldots \ldots \ldots \ldots$ & $\ldots$ & $\ldots$ & $\ldots$ & $\ldots$ & $\ldots$ & $\ldots$ & 3 & 3 & 3 & .. \\
\hline$-2 \ldots \ldots \ldots \ldots$ & $\ldots$ & $\ldots$ & $\ldots$ & 2.0 & 2.0 & 3 & 3 & 3 & 3 & .. \\
\hline$-1 \ldots \ldots \ldots \ldots$ & $\ldots$ & $\ldots$ & $\ldots$ & 2.0 & 2.1 & 2.1 & 3 & 3 & 3 & .. \\
\hline 0 & $\ldots$ & $\ldots$ & 2.0 & 2.0 & 2.1 & 2.1 & 2.2 & 2.3 & 2.3 & .. \\
\hline 1.............. & $\ldots$ & $\ldots$ & 2.0 & 2.0 & 2.1 & 2.1 & 2.2 & 2.3 & 2.3 & .. \\
\hline $2 \ldots \ldots \ldots \ldots$ & $\ldots$ & $\ldots$ & 2.1 & 2.1 & 2.1 & 2.2 & 2.2 & 2.3 & 2.3 & .. \\
\hline $3 \ldots \ldots \ldots \ldots \ldots$ & $\ldots$ & $\ldots$ & $\ldots$ & $\ldots$ & $\ldots$ & $\ldots$ & $\ldots$ & $\ldots$ & $\ldots$ & .. \\
\hline
\end{tabular}

TABLE 3-Continued

Notes.-COSMOS-calibrated ZEST grid in each plane of constant value of $\mathrm{PC}_{3}$, going from $\mathrm{PC}_{3}=-2$ in the first panel of the table, to $\mathrm{PC}_{3}=3$ in the last panel. In each panel, we give for each $\mathrm{PC}_{1}, \mathrm{PC}_{2}$ the ZEST classification as type 1 , 2 , and 3 . For type 2 disk galaxies, we also present the bulgeness parameter associated with the cube.

(listed in col. [1]), in the linear combination that gives the direction of the specific PC (e.g., $\mathrm{PC}_{1}=-0.54 x_{1}+0.60 x_{2}-0.56 x_{3}+$ $\left.0.20 x_{4}+0.02 x_{5}\right)$.

In Figure 2 we show the fraction of variance as a function of the corresponding PC. Circles refer to the PCA applied to the COSMOS galaxies in the considered $I_{\mathrm{AB}} \leq 24$ sample. Squares refer to the same analysis, performed, however, on only the COSMOS galaxies brighter than $I_{\mathrm{AB}}=22.5$. The horizontal line represents the value of the eigenvalues that would be expected if the five variables were uncorrelated. The comparison of the results for the $I_{\mathrm{AB}} \leq 22.5$ and the $I_{\mathrm{AB}} \leq 24$ samples demonstrates the stability of the analysis down to the faintest magnitudes in the sample.

As discussed in Appendix B, the scatter in the measured variables that define the PCs increases with increasing magnitude (the scatter at $I_{\mathrm{AB}}=24$ is twice the scatter measured at $I_{\mathrm{AB}}=$ 22). While the larger scatter in the data could potentially wash out correlations present in the original parameters, this effect is negligible down to the considered $I_{\mathrm{AB}}=24$ mag limit: e.g., the fraction of variance in $\mathrm{PC}_{1}$ is only $\sim 3 \%$ larger in the $I_{\mathrm{AB}} \leq 22.5$ sample relative to the $I_{\mathrm{AB}} \leq 24$ sample.

Several methods have been proposed in the literature to establish the number of PCs that are sufficient to fully describe the properties of the sample; all methods require some degree of judgment. For example Kaiser (1960) proposed the rule of thumb of rejecting all components that contain less power than the variance expected for uncorrelated variables (in our case of five variables, less than 20\%); Jolliffe (1972) adopted instead a lower threshold value. To classify galaxy structure, we will use the first three PCs; these explain $92 \%$ of the total variance. The ZEST classification grid is therefore constructed in a three-dimensional space; specifically, galaxies are ranked, according to their structural properties, in unit cubes of the $\mathrm{PC}_{1}-\mathrm{PC}_{2}-\mathrm{PC}_{3}$ ZEST space.

\subsubsection{Morphological Classification of the $P C_{1}-P C_{2}-P C_{3}$ Unit Cubes}

COSMOS galaxies with different structural properties occupy different regions of the $\mathrm{PC}_{1}-\mathrm{PC}_{2}-\mathrm{PC}_{3}$ space. For example, $\mathrm{PC}_{1}$ is dominated by $C, M_{20}$, and $\mathrm{G}$. Highly negative values of $\mathrm{PC}_{1}$ are populated by highly centrally concentrated galaxies. ${ }^{16} \mathrm{PC}_{2}$ is, on the other hand, dominated by ellipticity and asymmetry: round 


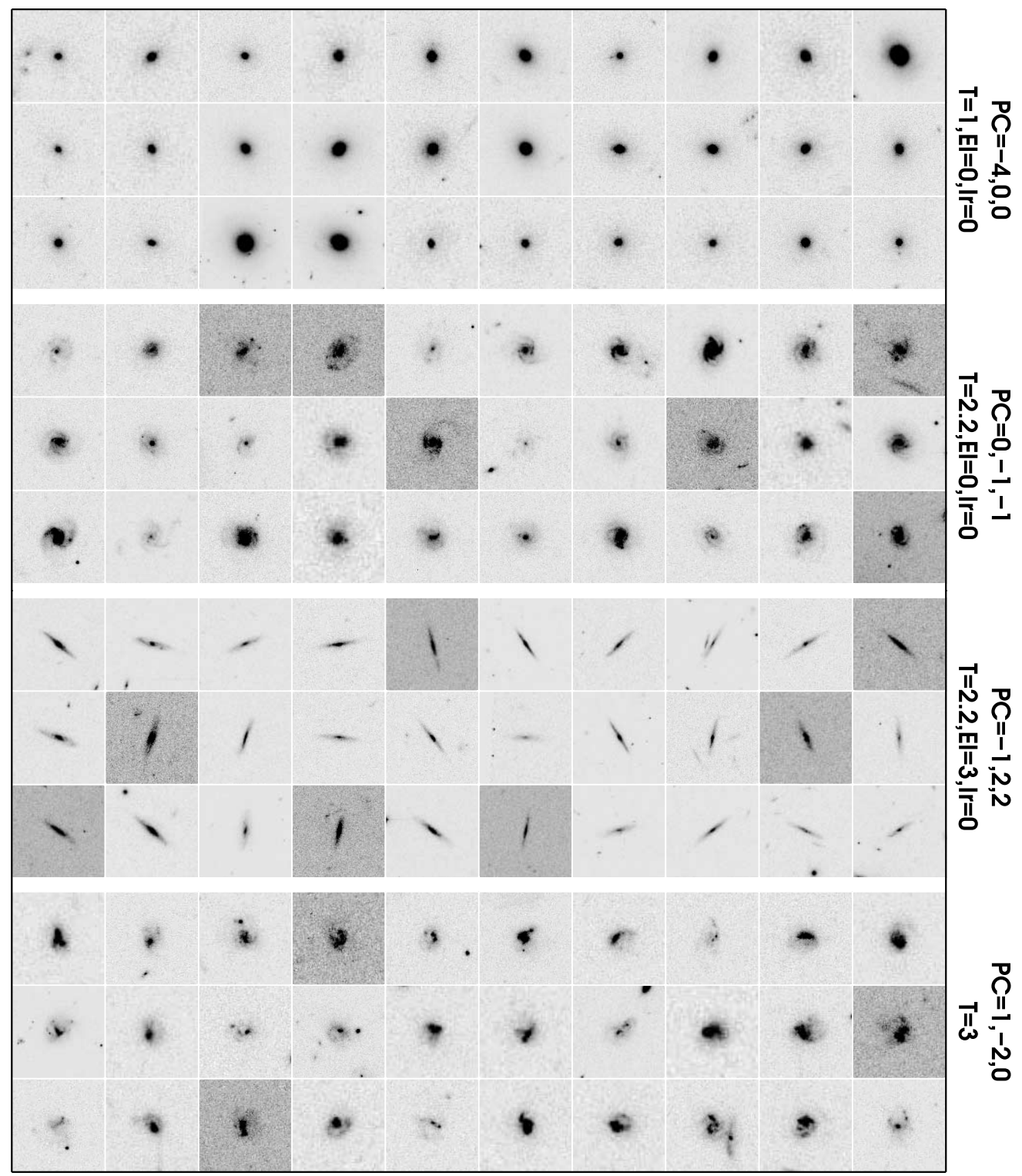

Fig. 3.-Illustration of the power of the ZEST grid to separate COSMOS galaxies with different structural properties in the three-dimensional $\mathrm{PC}_{1}-\mathrm{PC}_{2}-\mathrm{PC}_{3}$ space. Shown are four separate unit cubes of $\mathrm{PC}_{1}-\mathrm{PC}_{2}-\mathrm{PC}_{3}$, centered around the values reported in the labels. In every unit cube, the few galaxies are representative of the population of objects of that specific bin.

asymmetric objects are found at negative values of $\mathrm{PC}_{2}$, and symmetric flattened systems are preferentially located at high positive values of $\mathrm{PC}_{2} . \mathrm{PC}_{3}$ is also mostly a combination of asymmetry and ellipticity, but in $\mathrm{PC}_{3}$ these parameters contribute both with positive weights to the absolute value of $\mathrm{PC}_{3}$. Highly asymmetric and elongated objects are thus located at high values of $\mathrm{PC}_{3}$.

To associate a (dominant) morphological class to different regions of the $\mathrm{PC}_{1}-\mathrm{PC}_{2}-\mathrm{PC}_{3}$ space, the latter was partitioned into a regular three-dimensional grid with unit steps in each of the coordinates, and all galaxies in our COSMOS sample within each unit $\mathrm{PC}_{1}-\mathrm{PC}_{2}-\mathrm{PC}_{3}$ cube were visually inspected. Each unit cube was then assigned a morphological type $(T=1,2$, or 3 for early-type, disk, and irregular galaxies, respectively; face-on S0 galaxies would of course be found in cubes classified as $T=1$, while more-inclined S0 galaxies would be in cubes classified as $T=2$ ) and a clumpiness parameter (in unit steps, ranging from 0 for smooth surface density distributions, to 2 for very clumpy morphologies). For galaxies of $T=1$ or 2 , we furthermore as- signed an elongation parameter (in unit steps, from 0 for face-on galaxies to 3 for edge-on galaxies), and an irregularity parameter (in unit steps, ranging from 0 for regular surface density distributions to 2 for disturbed $T=1$ or 2 morphologies). A measure of the galaxy sizes (i.e., their Petrosian radii, see Appendix A.2) is also available for all ZEST-classified galaxies as a by-product of our analysis; see Appendix A.2).

\subsubsection{Bulge-to-Disk Ratios: Parametric Surface Brightness Fits}

To refine the ZEST structural classification of $T=2$ disk galaxies, we use additional information that we have available for a subsample of the COSMOS galaxies considered in this paper, i.e., the Galaxy Image 2D (GIM2D) single-Sérsic fits to the $I_{\mathrm{AB}} \leq$ 22.5 COSMOS galaxies of Sargent et al. (2007). In particular, we use the statistical distribution of Sérsic index $n$ within each unit cube of $\mathrm{PC}_{1}-\mathrm{PC}_{2}-\mathrm{PC}_{3}$ with a $T=2$ classification to assign a bulgeness parameter to each $T=2$ cube. Specifically, the $T=2$ unit cubes are split in four bins, i.e., $T=2.3,2.2,2.1$, and 2.0, depending on the value of the median Sérsic index $n$ of the galaxies 


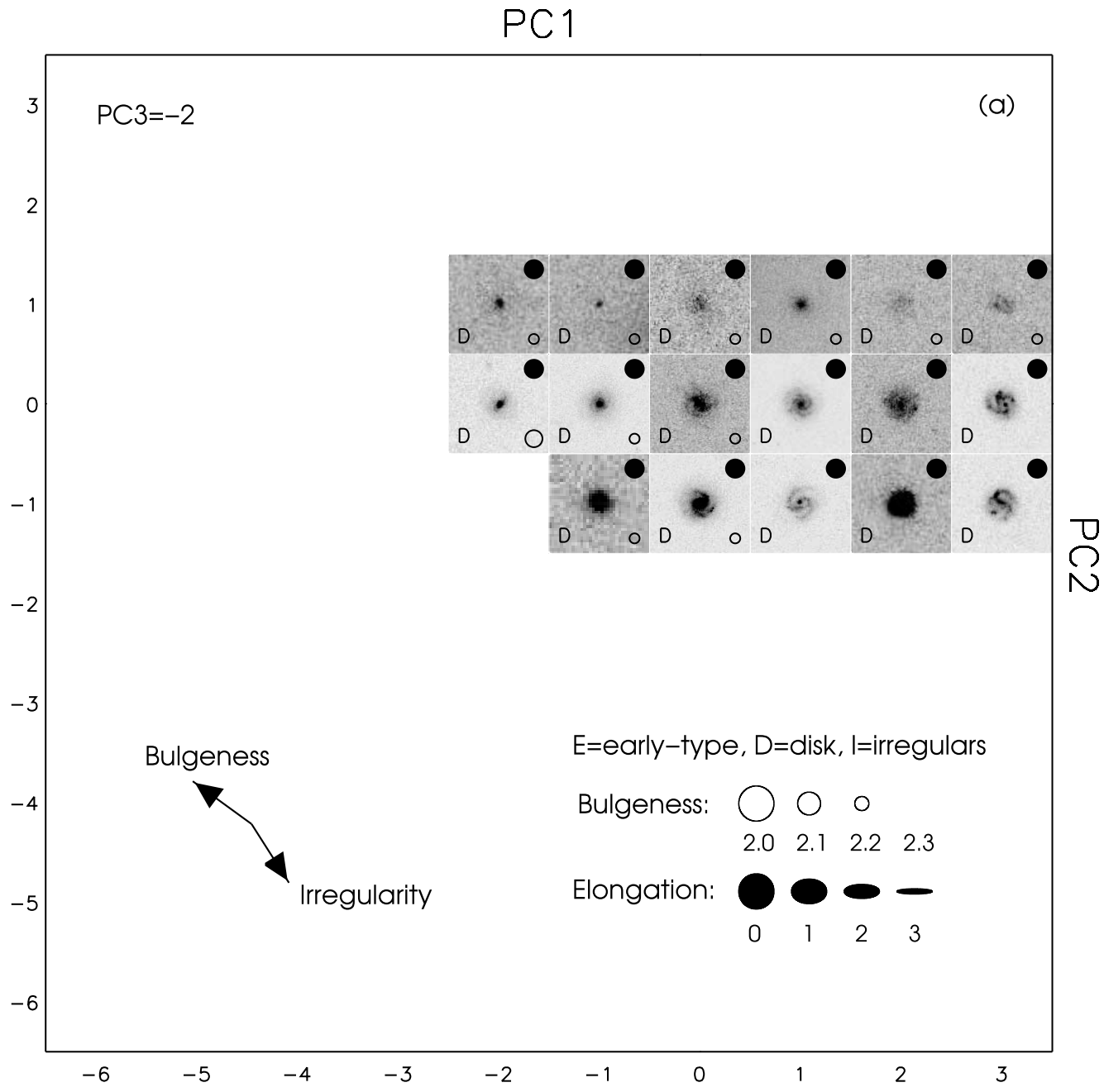

Fig. 4.- Stamp of a representative galaxy shown for each $\mathrm{PC}$ cube, arranged in planes of constant $\mathrm{PC}_{3}$ in six different panels $(a)-(f)$. In particular the panels show sequentially the $\mathrm{PC}_{3}=-2,-1,0,1,2,3$ planes. Galaxy structural properties — and thus galaxy types - change smoothly through the $\mathrm{PC}_{1}-\mathrm{PC}_{2}-\mathrm{PC}_{3}$ space.

in that cube $\left(T=2.3,2.2,2.1\right.$, and 2.0 for $0<n_{\text {med }}<0.75$, $0.75 \leq n_{\text {med }}<1.25,1.25 \leq n_{\text {med }}<2.5$, and $n_{\text {med }} \geq 2.5$, respectively). This refinement of the type-2 ZEST classification grid can be interpreted to correspond to a four-bin classification of disk galaxies in terms of their B/Ds, with the $T=2.0,2.1$, 2.2 , and 2.3 cubes hosting galaxies with decreasing $\mathrm{B} / \mathrm{D}$ (type $2.0=$ bulge-dominated galaxies, including relatively inclined S0 galaxies, and type 2.3 = bulgeless disks).

\subsection{Summary: The ZEST $P C_{1}-P C_{2}-P C_{3}$ Classification Scheme and Grid}

We summarize the final ZEST classification scheme in Table 2 and the COSMOS-calibrated ZEST grid in Table 3.

To show the performance of ZEST in disentangling galaxies with different structural properties, we plot in Figure 3 a representative selection of the $I_{\mathrm{AB}} \leq 24$ COSMOS galaxies that occupy four different unit cubes of $\mathrm{PC}_{1}-\mathrm{PC}_{2}-\mathrm{PC}_{3}$. Furthermore, in Figure 4 we show, in sequential planes of constant $\mathrm{PC}_{3}=$ $-2,-1,0,+1,+2,+3$, a representative galaxy in each of the $\left(\mathrm{PC}_{3}-\right) \mathrm{PC}_{1}-\mathrm{PC}_{2}$ unit bins. Arrows in the bottom left corners of each panel (i.e., $\mathrm{PC}_{3}=$ constant plane) indicate the directions of the steepest (positive) variation for the quantities specified as labels of the arrows; e.g., arrows labeled as "bulgeness" or "irregularity," respectively, show the direction, across the given
$\mathrm{PC}_{3}=$ constant plane, of the maximum increase of the "degree of bulgeness" and of the irregularity of the galaxies populating that plane.

In Figure 5 we summarize the COSMOS-calibrated ZEST classification grid in a schematic way. In each $\mathrm{PC}_{3}=$ constant plane, different symbols represent the different morphological types, elongation, and bulgeness parameter. As indicated in the figure, ellipses represent the $T=1$ early-type galaxies; concentric double circles indicate $T=2$ disk galaxies, and stars represent $T=3$ irregular galaxies. The size of the internal ellipse of $T=2$ galaxies is proportional to their bulgeness parameter. The continuity of properties in the $\mathrm{PC}_{1}-\mathrm{PC}_{2}-\mathrm{PC}_{3}$ space is immediately evident from Figure 5. For example, the transition from early-type morphologies $(T=1)$, to bulge-dominated disks $(T=2.0)$, to puredisk galaxies $(T=2.3)$ is smooth both across the $\mathrm{PC}_{1}-\mathrm{PC}_{2}$ planes and along the $\mathrm{PC}_{3}$ direction. The bulge-dominated galaxies are found preferentially at intermediate values of $\mathrm{PC}_{3}$ and, as expected, at low values of $\mathrm{PC}_{1}$.

\section{THE PERFORMANCE AND RELIABILITY OF ZEST}

Before demonstrating the robustness of the ZEST classification, it is important to stress that generally galaxy appearance depends on the rest-frame wavelength at which it is observed. Since only F814W ACS images are available for the COSMOS galaxies, it 


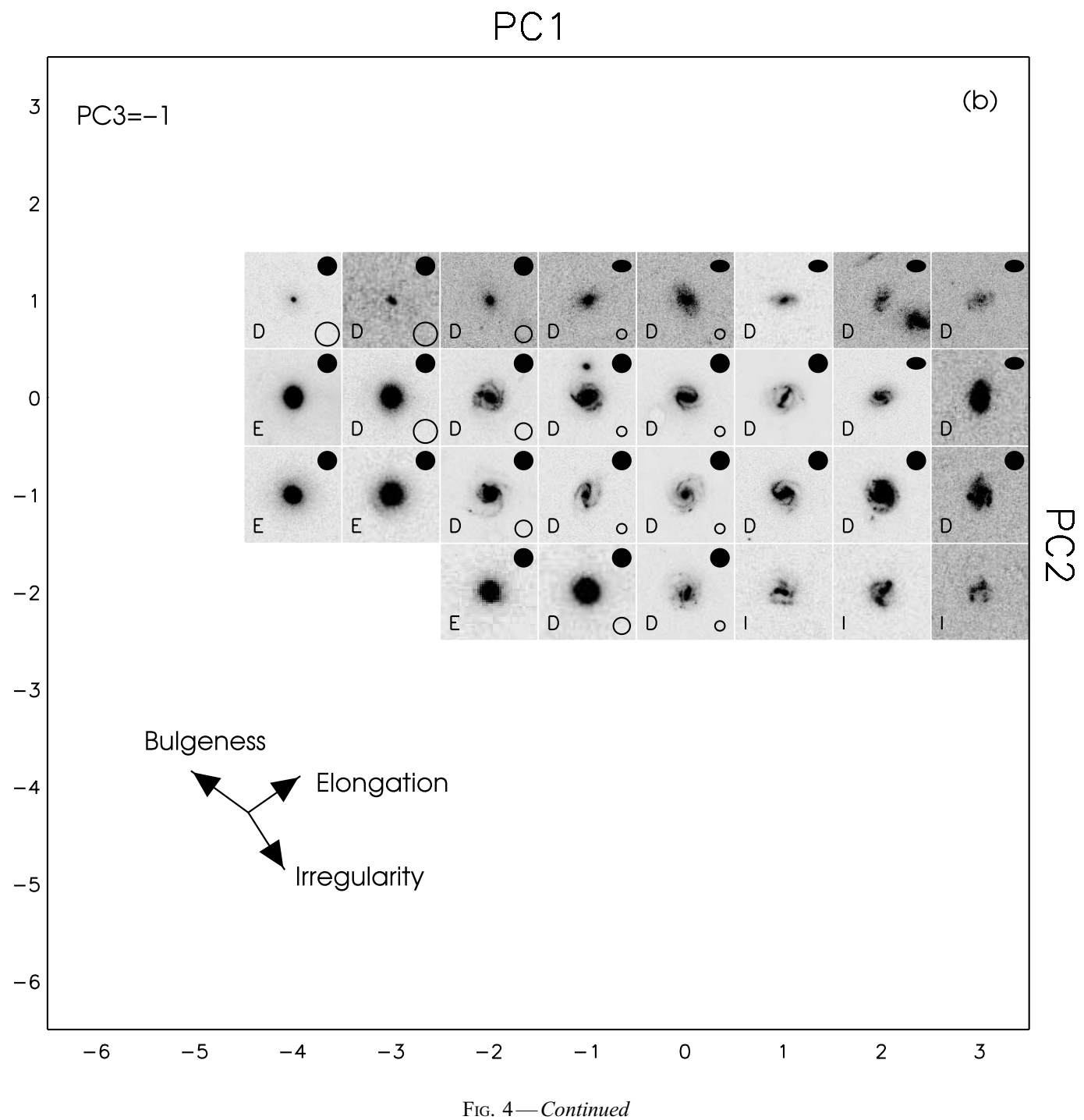

is important that any comparison of morphology for galaxies at different redshifts is treated with care. In the low-to-intermediate redshift regime $(0.2<z \leq 1.0)$ that is the focus of this paper, the central wavelength of the $\mathrm{F} 814 \mathrm{~W}$ filter covers the rest-frame 4000-6700 ^ window, where morphological $K$-corrections are negligible for most galaxies (see, e.g., Lotz et al. 2004; Cassata et al. 2005).

\subsection{Testing ZEST on $z=0$ Galaxies}

We assess the performance and reliability of the ZEST classification grid by applying it to the Frei et al. (1996) sample of $80 z=0$ representative galaxies that (1) have Hubble types available from the RC3 catalog (de Vaucouleurs et al. 1991), (2) have been observed (at the $1.1 \mathrm{~m}$ telescope of the Lowell Observatory) in the $B_{J}$ band $\left(\lambda_{\text {eff }}=4500 \AA\right)$ with a pixel scale of $1^{\prime \prime} .35$ pixel $^{-1}$, and (3) have a point-spread function (PSF) FWHM smaller than $5.0^{\prime \prime}$. We excluded from the analysis three galaxies for which the available Frei et al. images were too small to reliably get an estimate of the background. Frei et al.'s galaxies span Hubble types from $\mathrm{E}(T=-5)$ to $\mathrm{Sd}(T=10)$; they have been used as a $z=0$ benchmark for assessing galaxy morphologies at higher redshifts in a number of other works (e.g., Bershady et al. 2000; Simard et al. 2002; Lotz et al. 2004).
In Figure 6 we show the fraction of objects with a given RC3 classification ( $\mathrm{E}, \mathrm{S} 0-\mathrm{Sab}, \mathrm{Sb}-\mathrm{Scd}$, and $\mathrm{Sd}$ and later) that have $T=1,2.0,2.1,2.2,2.3$, and 3 ZEST classification. The comparison between the two classifications is excellent and highlights the power of the automatic ZEST classification scheme to recover the physically motivated Hubble types of galaxies.

There are only a few objects which have significantly different classifications between RC3 and ZEST. In Figure 7 we show the postage stamps of these most discrepant galaxies. A detailed analysis of these galaxies shows that

1. NGC 4621 and NGC 4564 , classified as -5 by the RC 3 but as type 2.0 by ZEST, have a disk component (Michard \& Marchal 1994; Scorza \& Bender 1995; Mizuno \& Oikawa 1996; Emsellem et al. 2004).

2. NGC 4710 has a ZEST $T=2.2$ and a Hubble type S0. As the image shows (see also Michard \& Marchal 1994), this edgeon galaxy displays a very bright ring and an important equatorial dust lane, which causes its visual classification to be highly uncertain: indeed, in the UGC catalog this galaxy is classified as an S0a.

3. The two galaxies classified as Type 1 by ZEST and with an $\mathrm{RC} 3$ classification of $\mathrm{S} 0-\mathrm{Sa}$ are relatively face-on galaxies. Their surface brightness is rather smooth with no visible spiral 


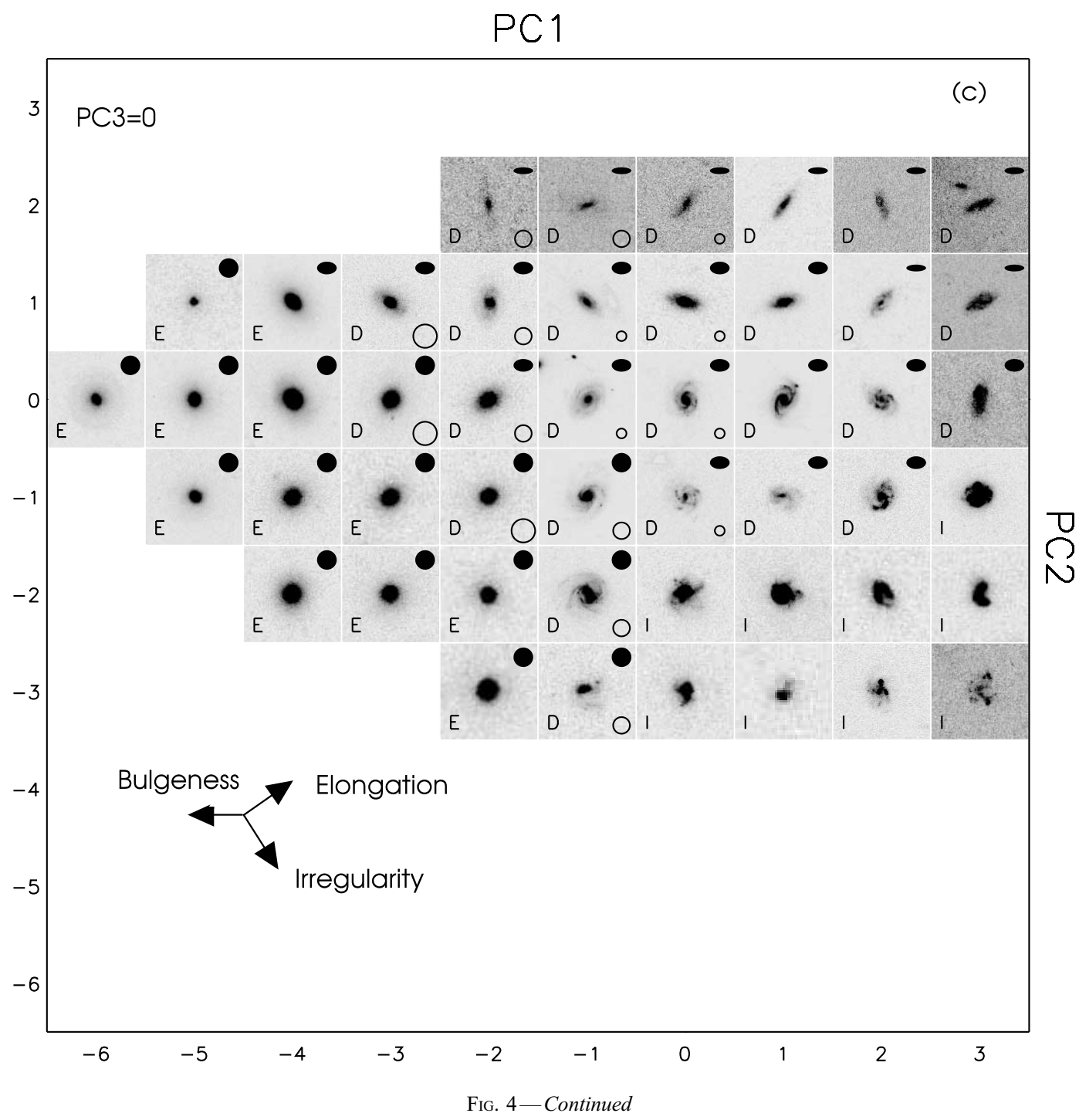

arms or star formation, with the exception of a smooth ring around NGC 4340.

4. NGC 4088, classified by ZEST as $T=3$ and by the RC3 as $\mathrm{Sb}-\mathrm{Scd}$, is described as an irregular and distorted spiral by Dahari (1985).

5. Finally, for the few galaxies which are classified by ZEST as bulge-dominated (type 2.0) galaxies and have RC3 classification of $\mathrm{Sb}-\mathrm{Scd}$, we performed a single Sérsic fit to their surface brightness profile. They have concentrated light distributions, with Sérsic index $n \geq 3$, which confirms our ZEST classification as type 2.0 galaxies.

\subsection{Error Bars in the ZEST Morphological Classification of COSMOS Galaxies}

In Appendix B we discuss in detail the uncertainties and the systematic errors in the measured structural parameters as a function of signal-to-noise ratio $(\mathrm{S} / \mathrm{N})$. To do so we use a sample of bright COSMOS galaxies, which are progressively dimmed to fainter magnitudes (lower $\mathrm{S} / \mathrm{N}$ ). Here we summarize to what extent the COSMOS-calibrated ZEST morphological classification grid is affected by the $\mathrm{S} / \mathrm{N}$ of the individual galaxies. We use the same sample of bright and progressively $\mathrm{S} / \mathrm{N}$-degraded test galaxies described in Appendix B and compute for each of them all the parameters involved in the ZEST classification, both on the original and on the progressively $\mathrm{S} / \mathrm{N}$-degraded images. The $\mathrm{PC}_{i}$ $(i=1,2,3)$ values are then computed for each galaxy at each $\mathrm{S} / \mathrm{N}$ level, and the ZEST morphological classification corresponding to the relevant unit cube of $\mathrm{PC}_{1}-\mathrm{PC}_{2}-\mathrm{PC}_{3}$ is assigned to each of the original and artificially dimmed galaxies. The change in galaxy type $(\Delta T)$ that occurs due to degraded $\mathrm{S} / \mathrm{N}$ is finally computed as a function of magnitude.

The ZEST classification is robust down to $I_{\mathrm{AB}}=24$. For magnitudes $I_{\mathrm{AB}} \leq 22.5$, more than $90 \%$ of galaxies do not change morphological class. The remaining few percent of galaxies changes morphological type by smoothly moving through the $\mathrm{PC}_{1}-\mathrm{PC}_{2}-\mathrm{PC}_{3}$ space; indeed, the change of ZEST morphological type with varying $\mathrm{S} / \mathrm{N}$ happens typically for galaxies that are originally classified in $\mathrm{PC}_{1}-\mathrm{PC}_{2}-\mathrm{PC}_{3}$ cubes that are close to a "type transition wall" in the $\mathrm{PC}$ space. The fraction of galaxies with $\Delta T=0$ remains larger than $\sim 75 \%$ down to magnitudes $I_{\mathrm{AB}}=23.0$, and even in the highest magnitude bin $(23.5<I<24)$, the fraction of galaxies with $\Delta T=0$ remains of order $65 \%$. This is illustrated in Figure 8 , where we show the distribution of the average absolute variation in $\mathrm{PC}_{i}$, namely, $\langle\Delta(\mathrm{PC})\rangle=\left(\sum_{i}\left|\mathrm{PC}_{i, f}-\mathrm{PC}_{i, 0}\right|\right) / 3$ (with $i=1$, 2,$3 ; \mathrm{PC}_{i, 0}$ the initial values of the $\mathrm{PC}_{i}$ 's; and $\mathrm{PC}_{i, f}$ the measured $\mathrm{PC}_{i}$ 's after $\mathrm{S} / \mathrm{N}$ degradation). Split in four magnitude bins (from 


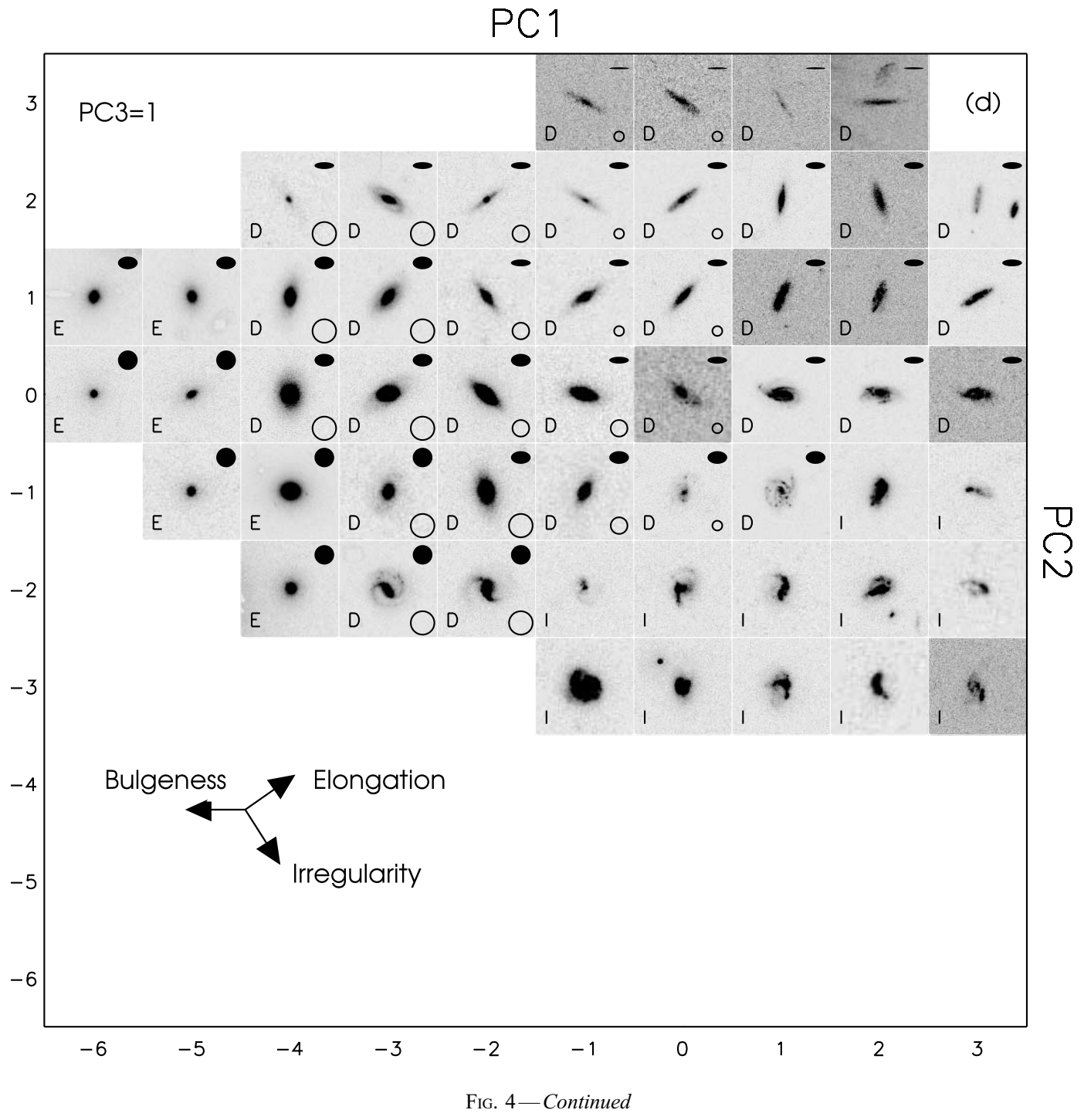

$I_{\mathrm{AB}}=22$, top left, down to $I_{\mathrm{AB}}=24$, bottom right), the solid histograms show the $\langle\Delta(\mathrm{PC})\rangle$ distribution for all galaxies in the considered magnitude bin, and the hatched histograms show the $\langle\Delta(\mathrm{PC})\rangle$ distribution for galaxies that change morphological class due to $\mathrm{S} / \mathrm{N}$ degradation.

Figure 9 shows a few examples of how galaxies move through $\mathrm{PC}$ space as the $\mathrm{S} / \mathrm{N}$ of the galaxy images decreases. Initial and final values for the $\mathrm{PC}_{i}$ coordinates of the example test galaxies are indicated at the beginning and end of the track that describes the movement, in the PC space of the specific test galaxy. Note that galaxies can have a $\langle\Delta(\mathrm{PC})\rangle$ as high as $\langle\Delta(\mathrm{PC})\rangle=2$, without changing their morphological class (as their path in $\mathrm{PC}_{i}$ space occurs within a region uniformly classified with a specific morphological type). As stressed above, virtually all galaxies that change morphological class are located, to start with, in bins of $\mathrm{PC}_{1}-\mathrm{PC}_{2}-\mathrm{PC}_{3}$ that border bins with a different morphological classification.

The most noticeable effect of $\mathrm{S} / \mathrm{N}$ degradation is a contamination, at the faintest magnitudes, of at most $\sim 30 \%$ from low-S/N early-type galaxies to the type 2 or 3 galaxy populations (see also Abraham et al. 1996; Lotz et al. 2004). This suggests that at most $30 \%$ of early-type galaxies at the faintest magnitudes could drop from the early-type sample and be misclassified as disk or irregular galaxies due to their lower $\mathrm{S} / \mathrm{N}$ values. We discuss in $\S 5.5$ the implications of this effect on the redshift evolution up to $z \sim 1$ of the LFs of COSMOS galaxies structurally classified with ZEST.

\section{THE ADVANTAGE OF THE ZEST CLASSIFICATION SCHEME OVER OTHER APPROACHES}

Popular classifications of (nearby and high-z) galaxies in the past few years have been typically based on combinations of two or three of the nonparametric diagnostics that are used in ZEST (e.g., Lotz et al. 2004; Ferreras et al. 2005 and references therein) or on a threshold in Sérsic index $n$ (most notably the SDSS defined early-type galaxies as the objects with $C \geq 2.87$ or $n \geq 2.5$, and late-type galaxies as all other galaxies). Such approaches can be seen as "lower dimensionality" projections of the ZEST grid and lead to galaxy samples that are affected by large contamination of systems with rather different structural properties.

As an example, in Figure 10 we show the two-dimensional planes defined by $\mathrm{G}$ and $M_{20}$ (top), $C$ and $M_{20}$ (middle), and $C$ and $A$ (bottom). All plots show the total density of galaxies in gray scale. In the different panels we indicate the region of space that is populated by a given ZEST morphological class; in particular, the color contours enclose $\sim 99 \%$ of the COSMOS galaxies with the specified ZEST morphological class. To avoid crowding we show in the left panels the location of the $T=1$ early-type galaxies (red contours), in the central panels the $T=2$ disk 


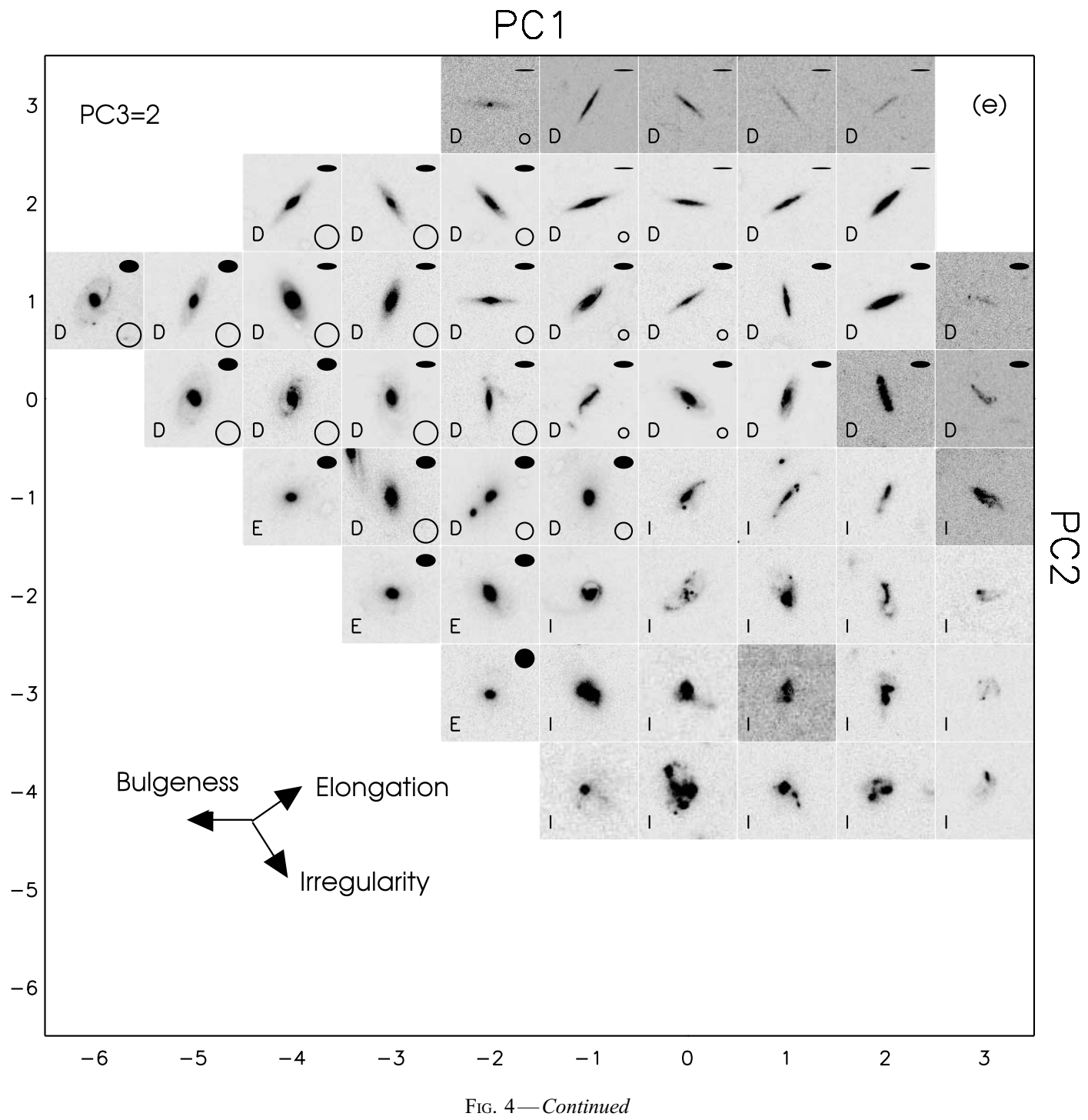

galaxies (blue contours, with decreasing shade of blue from the bulge-dominated $T=2.0$ galaxies to the $T=2.3$ bulgeless disks), and in the right panels the $T=3$ irregular galaxies (green contours).

It is clear from Figure 10 that there is a high level of contamination by different galaxy types in all regions of these twodimensional planes. For example, on the $C-M_{20}$ plane, $T=1$ early-type galaxies form a tight sequence, which is very distinct in the left panel of Figure 10; however, such a sequence includes not only the majority of $T=2.0$ bulge-dominated disk galaxies, but also a substantial fraction of lower B/D (i.e., $T=2.2,2.1$ ) galaxies. For example, if the galaxies that lie above the solid black line on the $C-M_{20}$ plane were classified as early types, ZEST would return the following galaxy population mixture in the selected region: $38 \%$ of desired $T=1$ early-type galaxies, $23 \%$ of $T=2.0$ galaxies, and $26 \%$ and $10 \%$ of $T=2.1$ and of $T=2.2$ galaxies, respectively. Even summing up together the $T=1$ and 2.0 galaxies (which might be desired for some science applications), the selected sample would still be affected by a contamination of order $40 \%$ contributed by lower B/D galaxies. Similar levels of contamination are found when the other twodimensional planes of Figure 10 or a simple cut in Sérsic index $n$ are used to morphologically classify galaxies, as has been done in most of the previous literature.

\section{A FIRST APPLICATION OF ZEST: THE EVOLUTION SINCE $z \sim 1$ OF THE LFs OF MORPHOLOGICAL EARLY-TYPE, DISK, AND IRREGULAR COSMOS GALAXIES}

We derive the rest-frame $B$-band LFs of ZEST-classified COSMOS galaxies brighter than $I=24$ (see Appendix A.1 for details on the COSMOS sample). Given the large number statistics of (the fraction of survey area that we are considering for) COSMOS, we can compute the $\operatorname{LF} \Phi(M, z, T)$ of each morphological class in four different redshift intervals.

We use the Zurich Extragalactic Bayesian Redshift Analyzer (ZEBRA) maximum likelihood photometric redshifts of Feldmann et al. (2006) to derive the COSMOS LFs. These photometric redshifts have an accuracy of $\Delta z /(1+z) \sim 0.03$ in comparison with the zCOSMOS spectroscopic redshifts of $I_{\mathrm{AB}} \leq$ 22.5 galaxies (Lilly et al. 2007); the accuracy of the ZEBRA redshifts degrades to $\Delta z /(1+z) \sim 0.06$ down to our magnitude limit of $I_{\mathrm{AB}}=24$ (see Appendix A.3 for details). The application of ZEBRA to our sample shows some dependence of the resulting photometric redshifts on whether small (of order $\sim 0.05$ mag or smaller) systematic offsets, which are detected by the code, are applied to the photometric calibration of the COSMOS Subaru data. This has, however, no substantial impact 


\section{PC1}

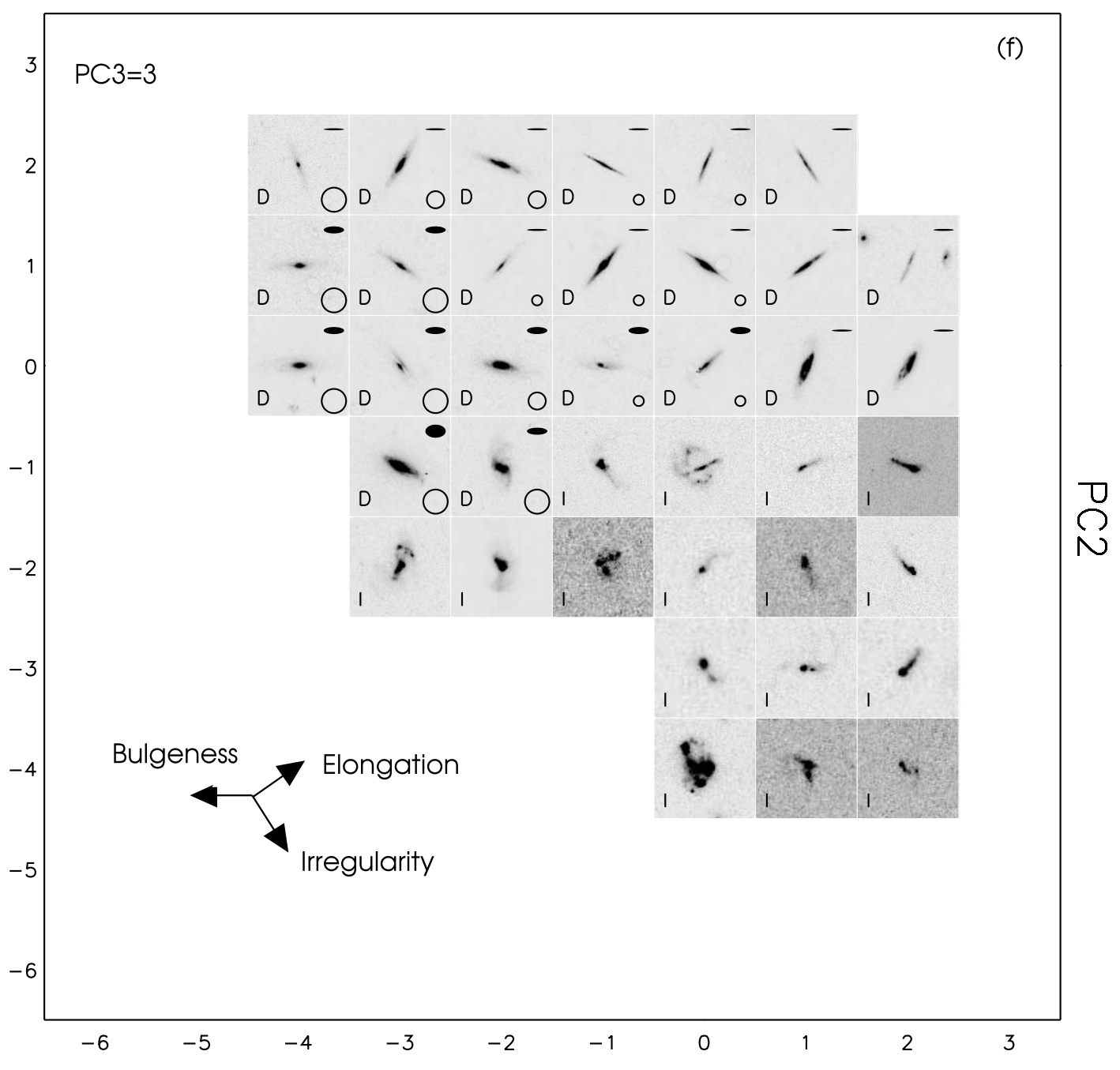

FIG. 4-Continued

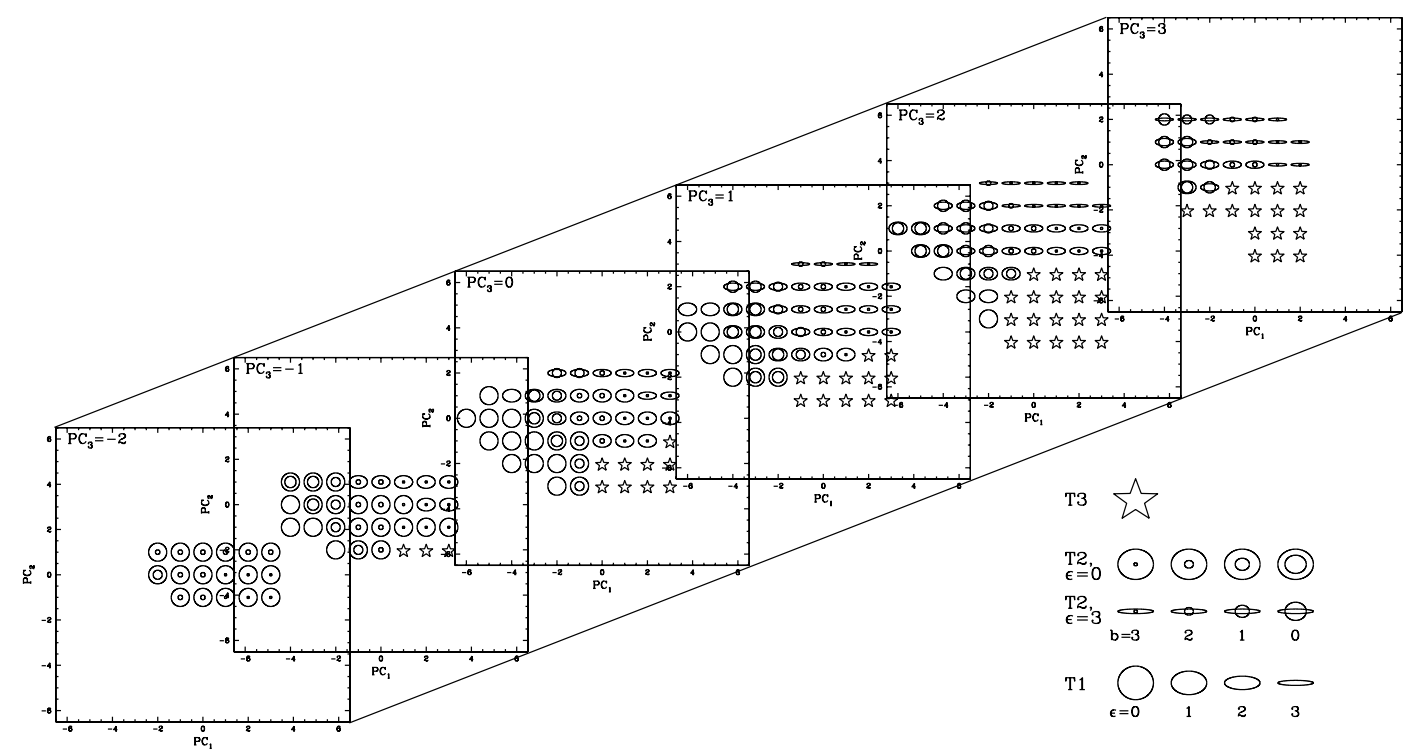

FIG. 5.-ZEST morphological classification. Shown are slices of the $\mathrm{PC}$ space at constant value of $\mathrm{PC}_{3}$. Each slice shows $\mathrm{PC}_{1} \mathrm{vs} \mathrm{PC}_{2}$; the value of $\mathrm{PC}_{3}$ increases from bottom left to top right. In each unit cube the symbol indicates the ZEST classification associated with that cube (according to the legend shown in the bottom right corner of the figure). In particular, stars represent $T=3$ galaxies, and single ellipses represent $T=1$ galaxies. The double ellipses indicate $T=2$ galaxies, for which the size of the inner ellipse increases for increasing bulgeness parameter. 


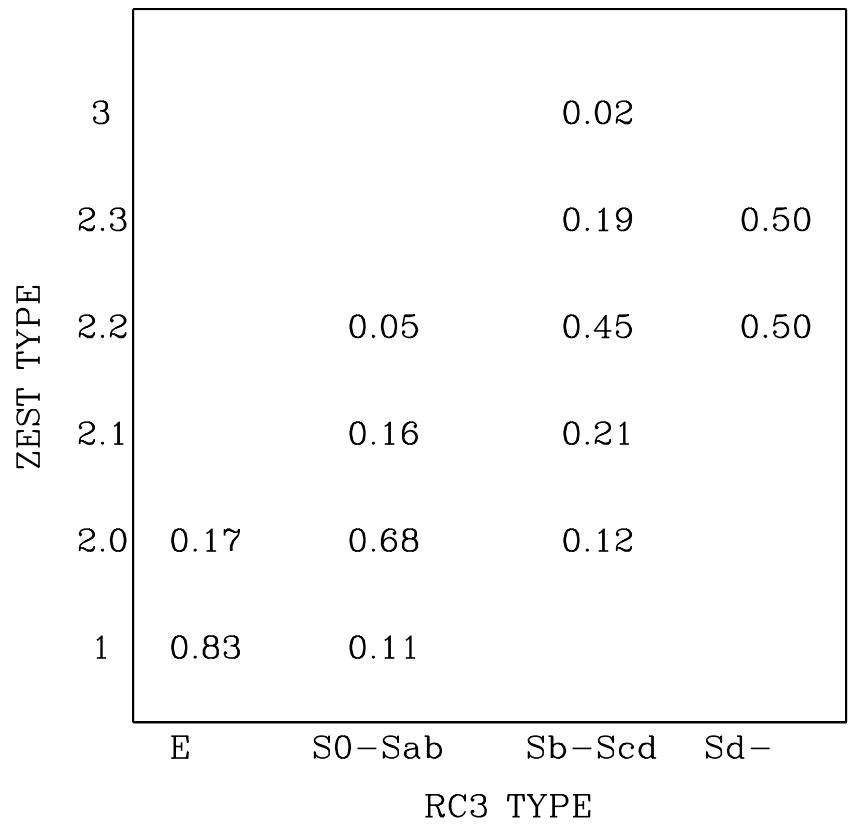

FIG. 6.-Fraction of objects with a given RC3 classification ( E, S0-Sab, SbScd, and Sd and later) that have $T=1,2.0,2.1,2.2,2.3$, and 3 ZEST morphological type.

on our resulting LFs, which we present below as computed both with and without corrections for these photometric offsets.

The final $I_{\mathrm{AB}} \leq 24$ COSMOS sample that we study below consists of 30,760 galaxies classified with ZEST as $T=1$ (2497 objects), $T=2$ (26,873 objects), and $T=3$ (1390 objects), which have photometric redshifts in the range $0.2<z \leq 1.0$.

\subsection{Luminosity Functions: Definitions}

We estimated the rest-frame $B$ galaxy LF in different redshift bins, using the $1 / V_{\max }$ estimator (Schmidt 1968; Felten 1976). According to the original $1 / V_{\max }$ formalism, the number of galaxies per unit comoving volume in the range of absolute magnitudes $d M$, at redshift $z$ and morphological class $T$ can be written as

$$
\int \Phi(M, z, T) d M=\sum \frac{1}{V_{\max , i}}
$$

where the sum is over all galaxies in the specific range of redshift, absolute magnitude, and morphological class. The quantity $V_{\max , i}$ is the maximum comoving volume within which the galaxy $i$ could still be detected according to the apparent magnitude limits of the survey, which, in our case, are given by $16 \leq I \leq 24$. The $V_{\max , i}$ is computed for each galaxy according to

$$
V_{\max , i}=\Omega \int_{\max \left(z_{L}, z_{16}\right)}^{\min \left(z_{U}, z_{24}\right)} \frac{d V}{d z} d z,
$$

where $z_{U}$ and $z_{L}$ are the upper and lower redshift of the considered redshift bin, and $z_{24}$ and $z_{16}$ are the redshifts at which a galaxy of a given rest-frame $B$-band absolute magnitude and a given spectral energy distribution (SED) would have $I$-band apparent magnitude of 24 and 16, respectively. The values of $z_{24}$ and $z_{16}$ were computed, for each galaxy, by taking into account the $k$-correction resulting from the best-fit SED provided by the ZEBRA fit. No evolutionary correction was applied. The quantity $\Omega$ is the effective area of the survey, corresponding to $0.74 \mathrm{deg}^{2}$.

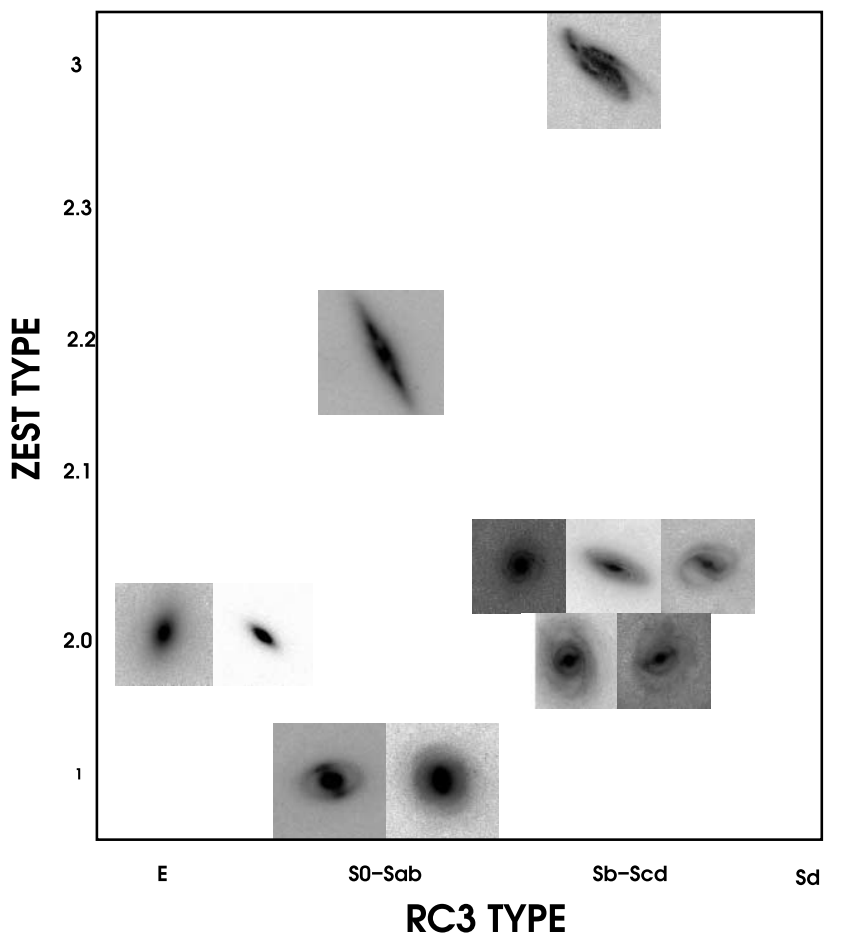

FIG. 7.- Images of the $z=0$ Frei et al. (1996) galaxies with relatively discrepant ZEST and RC3 classifications.

The Poissonian errors from galaxy counts on $\Phi(M, z, T)$ are given by $\sigma_{\Phi}=\left(\sum 1 / V_{\max , i}^{2}\right)^{1 / 2}$.

Since we are working with a magnitude-selected rather than a volume-limited sample, the highest and lowest $B$-band magnitudes at each redshift depend on the galaxy SED. This is evident in Figure 19 of Appendix A.4, where we show the color $k$-correction (i.e., difference between the observed $I$ - and the $B$-band rest-frame magnitude), as a function of redshift, for templates of different photometric type (from early-type galaxies to starbursting galaxies). The effect vanishes at redshift $z=0.8$, where the central wavelength of the F814W filter matches exactly the rest-frame $B$ band. Since correcting for this bias needs an a priori assumption on the color distribution of the nondetected galaxies, we limit the computation of the LF in each redshift bin to the luminosity range for which we are complete, regardless of galaxy colors. The used magnitude ranges are listed in Table 4.

\subsection{Corrections for Missing Sources}

About $2.4 \%$ of the ACS-detected galaxies do not have a match in the ground-based catalog; therefore, no information on their SEDs - and thus photometric redshifts - can be derived. Furthermore, about $2 \%$ of the ACS-detected sources are in close pairs, and, particularly, are at a distance from each other smaller than $0.6^{\prime \prime}$; therefore, these sources are detected as a single object in the ground-based images (see Appendix A.1 for details). The photometric redshifts estimated for these objects are thus unreliable, even for those pairs that are really physically associated and thus at the same distance, since the ground-based images mix the light from both galaxies, which could have very different stellar population. Therefore, these galaxies in close pairs also need to be excluded from the analysis of the COSMOS LFs.

In Figure 11 we show the $I$-band magnitude distribution of the ACS galaxies with no detection in the ground-based catalog (top) and the magnitude distribution of the ACS galaxies for which 

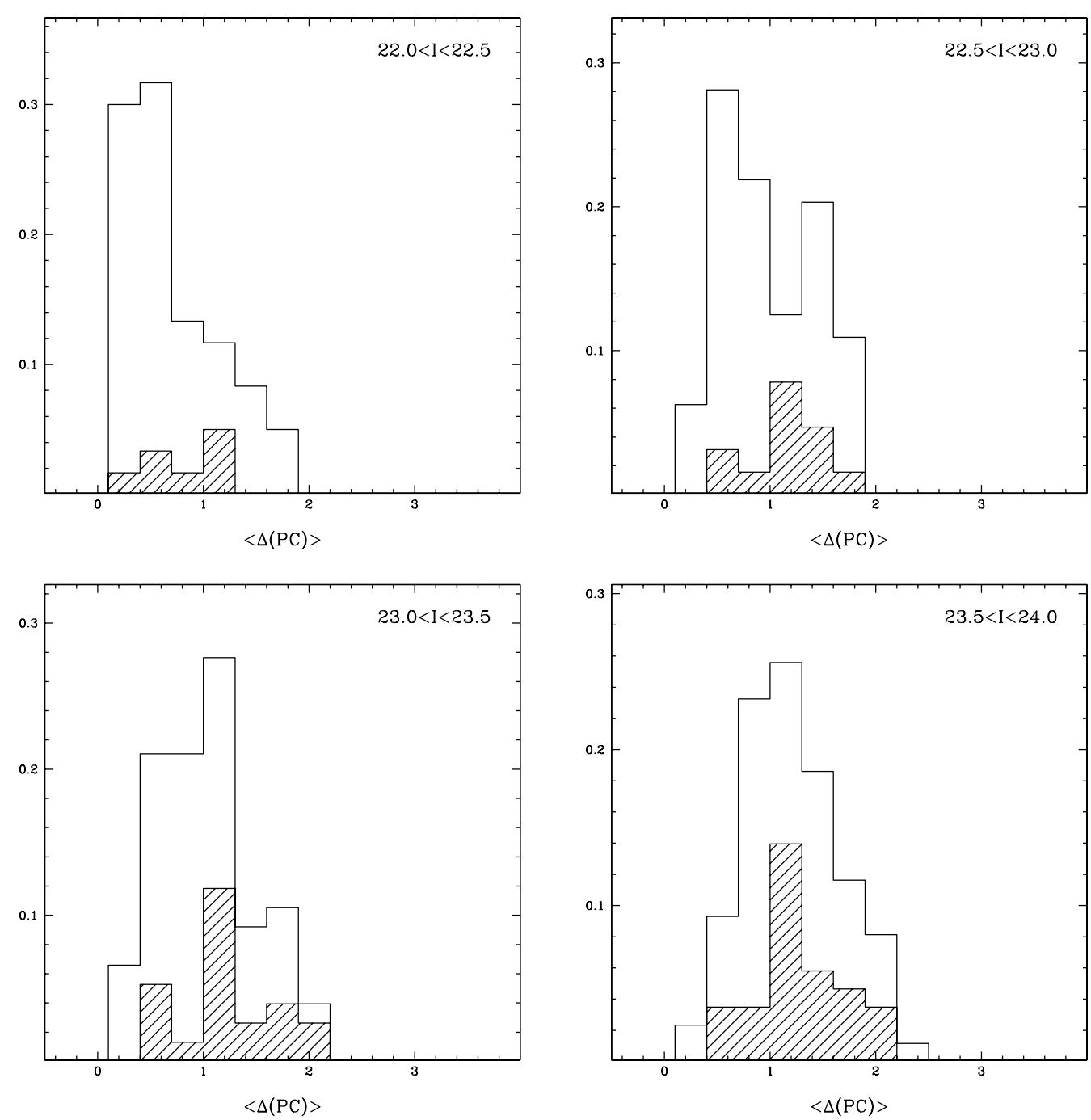

FIG. 8.-Solid histograms: Distribution of $\left.\langle\Delta(\mathrm{PC})\rangle=\sum_{i}\left|\mathrm{PC}_{i}-\mathrm{PC}_{i, 0}\right|\right) / 3$, with $i=1,2,3 ; \mathrm{PC}_{i, 0}$ the initial values of the $\mathrm{PCs}$; and $\mathrm{PC}_{i}$ the measured $\mathrm{PCs}$ after $\mathrm{S} / \mathrm{N}$ image degradation of a set of $17.5<I<18$ COSMOS galaxies. We show four different magnitude bins for the $\mathrm{S} / \mathrm{N}$-degraded galaxies, from $22.0<I<22.5$ (top left) down to $23.5<I<24.0$ (bottom right). The hatched histograms show the $\langle\Delta(\mathrm{PC})\rangle$ distribution for the galaxies with $\Delta T \neq 0$.

the same ground-based identification was associated with multiple ACS sources (bottom). Both distributions are normalized to the number of galaxies in the total sample in each magnitude bin. The fraction of missed objects stays relatively constant down to a I-band magnitude of $\sim 22$ but then increases toward fainter magnitudes. Although the fraction of missed objects is small, its dependence on the observed I-band magnitude may introduce biases in the computation of the LF.

To assess the potential impact of such biases, we therefore computed two versions of the LFs, respectively assuming that

1. All galaxies with no redshift are outside the $0.2<z \leq 1.0$ range. We refer to these LFs as the uncorrected LFs.

2. The missing galaxies have the same redshift distribution as the galaxies with an available redshift. In this case we can calculate the statistical weight $\left(\chi_{i}\right)$ needed to correct the $V_{\max }$ value of galaxies with a known redshift. We calculate $\chi_{i}$ as a function of the observed $I$-band magnitude, following the approach used by Willmer et al. (2006). We consider magnitude bins of $0.5 \mathrm{mag}$, and for each magnitude bin we compute (1) the number of galaxies with photometric redshift in the considered redshift range ( $N_{z}$, with $0.2<z \leq 1.0$ ); (2) the number of galaxies with $z>z_{h}$
$\left(N_{z h}\right.$, with $\left.z_{h}=1.0\right)$; and (3) the number of galaxies with $z>z_{l}$ $\left(N_{z l}\right.$, with $\left.z_{l}=0.2\right)$. Under the assumption that objects without a redshift estimate have the same redshift distribution of the entire sample, the probability for a galaxy of magnitude $I_{i}$ of being in the redshift range $0.2<z \leq 1.0$ is given by the number of galaxies with good redshift estimates in that range, divided by the sum of the number of objects with good redshift plus the number of objects with redshift both lower and higher than the considered limits, i.e., $P_{i}=N_{z} /\left(N_{z}+N_{z h}+N_{z l}\right)$. Finally, the weight $\chi_{i}$ of each galaxy with estimated redshift is the sum of the $P_{i}$ of all galaxies in the relevant magnitude bin, divided by the number of galaxies in that bin with $0.2<z \leq 1.0$ (i.e., $\sum P_{i} / N_{z}$ ). The $V_{\max , i}$ that is associated with each galaxy, weighted to account for objects with no available redshift, is simply $\chi_{i} / V_{\max , i}$, and the Poissonian errors on the LF, in each magnitude bin, are given by $\sigma_{i}^{2}=\sum \chi_{i} / V_{\max , i}^{2}$. In the following we will refer to the so-obtained LFs as the corrected LFs.

In Figure 12 we show the LFs of the total sample of $I_{\mathrm{AB}} \leq 24$ COSMOS galaxies, as well as the LFs for the different ZEST types. Specifically, we show the comparison between the uncorrected (dotted line) and the corrected (stars) LFs. The figure shows, from 

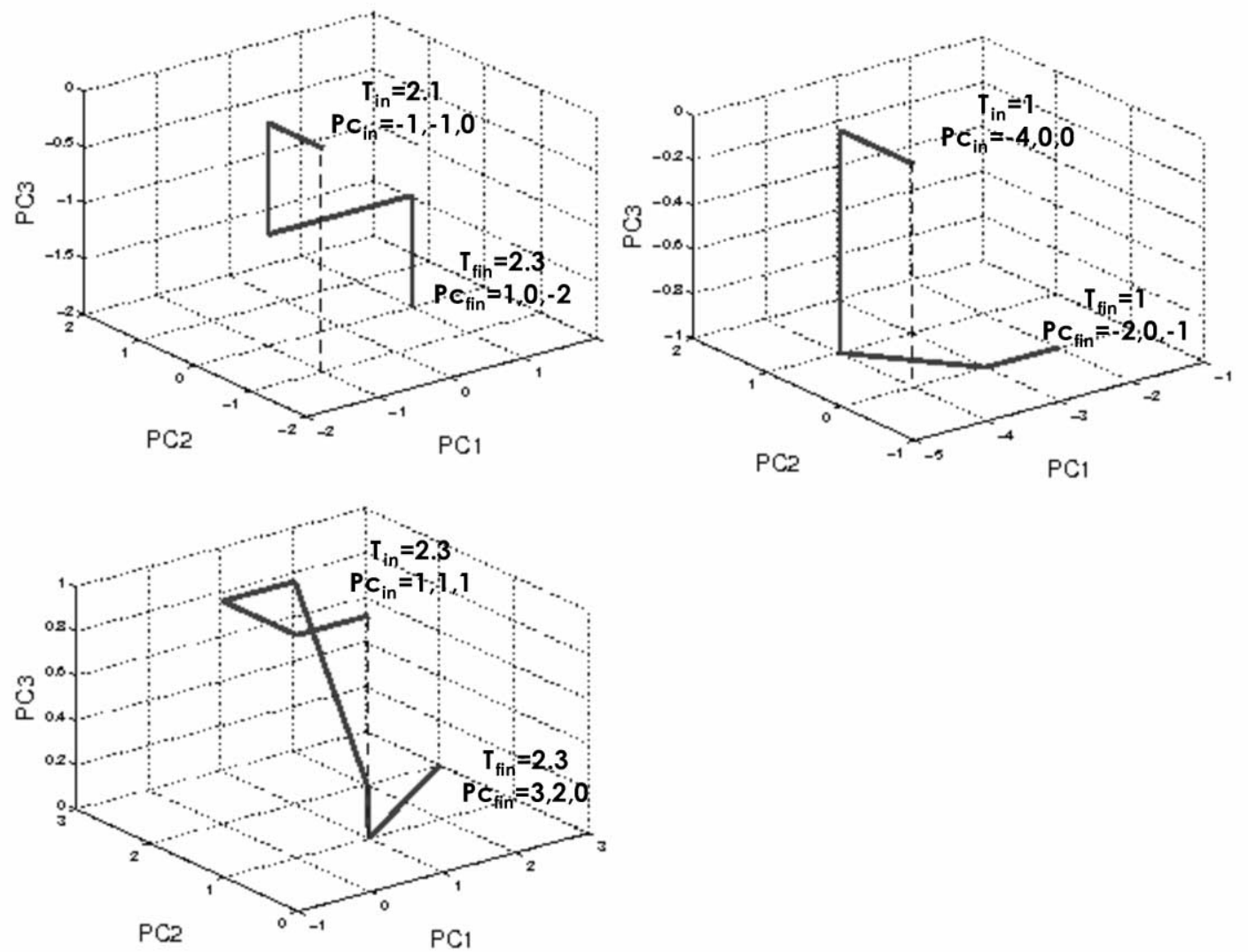

FIG. 9.- Three examples of how galaxies move in the PC space as the $\mathrm{S} / \mathrm{N}$ of the galaxy image decreases. The start and end coordinates in PC space are indicated ( $\mathrm{PCin}$ and PCfin, respectively). [See the electronic edition of the Supplement for a color version of this figure.]

top to bottom, the LF integrated over all morphological types and the LFs for the $T=1$ (early-type), $T=2$ (disk), and $T=3$ (irregular) galaxies, respectively. Each column shows the LFs calculated in different redshift bin: $0.2<z \leq 0.4$ (first column), $0.4<z \leq 0.6$ (second column), $0.6<z \leq 0.8$ (third column), and $0.8<z \leq 1.0$ (last column). The plotted error bars show the Poisson errors only. Uncertainties due to sampling variance are not included in the figures; these are discussed in $\S 5.4$. In each redshift bin, the LFs are shown down to the absolute $B$-band magnitude at which the sample is complete, regardless of galaxy colors, so that both red and blue galaxies are sampled in an unbiased way at each redshift, down to the faintest magnitude bin.

\subsection{Impact on the LFs of the Uncertainties in the Photometric Redshifts}

In order to check to what extent the limited redshift accuracy affects the derived LFs, we performed a set of simulations using the COSMOS mock galaxy catalogs of Kitzbichler et al. (2007); these are designed to reproduce in detail the COSMOS survey. From these mock catalogs, we first extracted all galaxies with observed magnitude $I_{\mathrm{AB}} \leq 24$, covering an area on the sky equal to that analyzed in this paper, and then generated 100 simulations of the galaxy catalog, perturbing each redshift with an error derived by randomly sampling a Gaussian with a $\sigma$ equal to that of the photometric redshift uncertainty in the relevant redshift bin. We considered $\sigma_{z} /(1+z) \sim 0.03$ for galaxies brighter than $I_{\mathrm{AB}}=22.5$ and $\sigma_{z} /(1+z) \sim 0.06$ for fainter objects (see Appendix A.3). The LFs of the original catalog and of the 100 resimulated samples were then calculated using the same procedure described in $\S 5.1$. The results of these tests are presented in Figure 13, where, in each panel, the solid line with Poissonian error bars represents the LF computed using the "true" redshifts and the diamonds represent the median of the 100 realizations. The shaded gray area associated with the simulated volume densities represents the 16th to 84th percentiles of the simulated distributions within each magnitude bin. Figure 13 clearly shows that the dominant effect of the redshift uncertainty is the systematic trend of (1) populating the bright end of the LF, where few or no galaxies are present in the original sample, and (2) slightly underestimating the density of galaxies around the knee of the LF (i.e., around $M_{*}$ ). However, even at the bright end of the LF, where the effect is the strongest, the real and the simulated LFs are well within $2 \sigma_{\Phi}$, implying that the uncertainty in the photometric redshifts does not affect our conclusions on the evolution of the LFs. The largest source of uncertainty, especially in the lowest redshift bin, is due to the small volume sampled by the bin, where the contribution from large-scale structure variation is significant. This explains the relative large difference in the LFs calculated from two independent mock catalogs (shown with dashed lines in Figure 13).

In Figure 12 we show, as stars, the corrected LFs derived adopting the ZEBRA photometric redshift calculated after correcting the photometric catalog for the small systematic offset, and, shaded in gray, the uncertainty on the LFs that arises when using the ZEBRA photometric redshift estimates obtained without correcting the photometric catalog (see Appendix A.3). Although the LFs estimated with the two sets of photometric redshifts differ somewhat (see Table 4 for quantitative differences in the corresponding Schechter fits), the differences do not substantially impact our conclusions. Furthermore, Figure 12 illustrates that the LFs derived with the two approaches described in $\S 5.2$ are in very good agreement, demonstrating that the results are independent of the (small) fraction of objects excluded for not having a photometric 


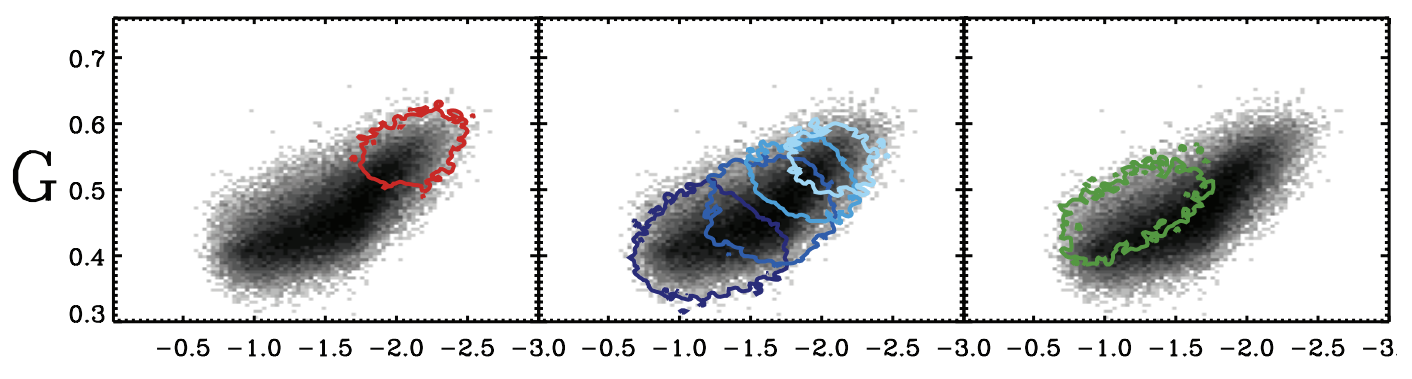

$\mathrm{M}_{20}$

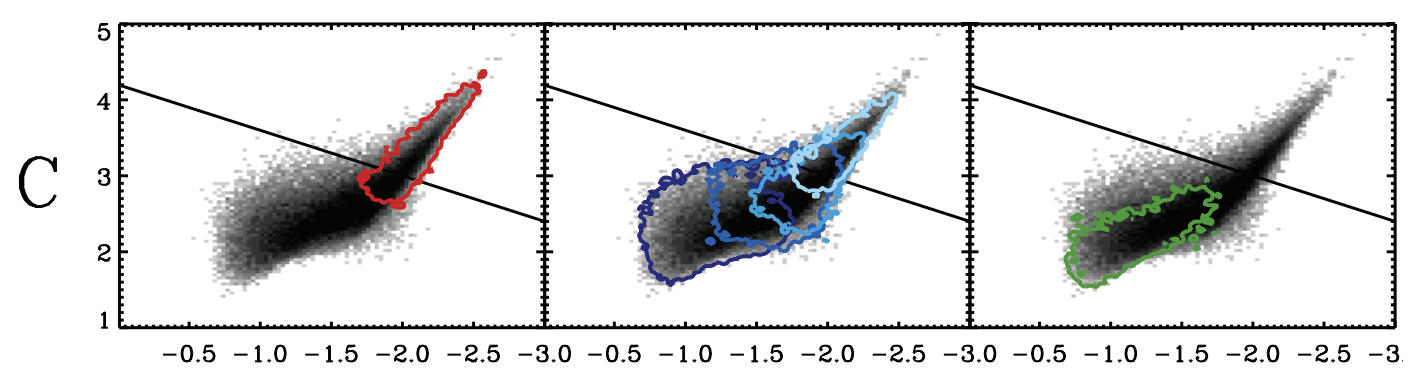

$\mathrm{M}_{20}$

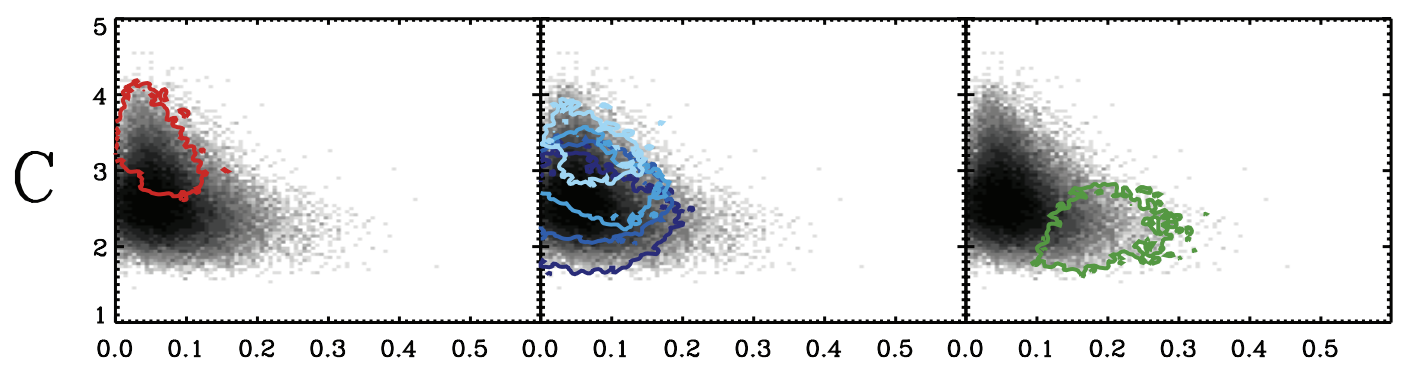

A

FIG. 10.-Distribution of our $\sim 56,000 I_{\mathrm{AB}} \leq 24$ COSMOS galaxies in the G- $M_{20}$ plane (top), $C-M_{20}$ plane (middle), and $C$ - $A$ plane (bottom). In each column we highlight the position of a different ZEST morphological class by drawing the contours enclosing $99 \%$ of the objects in that ZEST class. In the first, second, and third columns we show, respectively, $T=1,2$, and 3 galaxies (red, blue, and green contours). The blue color for $T=2$ galaxies ranges from dark blue for bulge-dominated $T=2.0$ galaxies to light blue for $T=2.3$ bulgeless disks.

redshift estimate. In the following we therefore focus our discussion entirely on the corrected LFs (stars in Fig. 12).

\subsection{Results: The Evolution of the LFs for the Different Morphological Classes}

The first column of Figure 12 shows that, in the lowest redshift bin, the shape of the LF varies significantly for different morphologically selected galaxy samples. In particular, the LFs of the global galaxy sample and of $T=2$ disk galaxies keep increasing toward faint magnitudes and have a rather similar behavior. On the other hand, the LFs of the $T=1$ early-type galaxy population and of the $T=3$ irregular galaxies appear, within the errors, to almost flatten for magnitudes $M_{B}>-19.5$.

We note that our COSMOS LFs computed in the first redshift $\operatorname{bin}(z=0.2-0.4)$ are rather susceptible to the effect of large-scale structures, as the size of the studied COSMOS field at $z=0.3$ is only $\sim 20$ comoving Mpc. To quantify this effect, we compare the LF for the global galaxy population with the LFs derived in the same redshift bin by other surveys. A fit with a Schechter function to the COSMOS LF in this redshift bin gives the following best-fit parameters: $\Phi_{*}=0.005 \pm 0.001 \mathrm{Mpc}^{-3}, M_{B, *}=$ $-21.0 \pm 0.2$, and $\alpha=-1.26 \pm 0.15$. The faint-end slope $\alpha$ is in good agreement with the value recently published by Willmer et al. (2006) $\alpha_{\mathrm{W}}=-1.3$ on the basis of the DEEP2 spectroscopic survey (Davis et al. 2003). The DEEP2 LF is based on spectroscopic redshifts; therefore, the consistency of the DEEP2 LFs with that which we have presented highlights the reliability of our photometric redshift estimates. In the $0.2<z<0.4$ bin, the comparison between our value of $\Phi_{*}$ and those derived from other surveys demonstrates that the average density in the COSMOS field is systematically higher than in other studies at similar redshifts. For example, Willmer et al. (2006) find $\phi_{*}=$ 26.39-1.81 $\times 10^{-4} \mathrm{Mpc}^{-3}$, and Wolf et al. (2003) find $\phi_{*}=$ $18.27 \pm 12.98 \times 10^{-4} \mathrm{Mpc}^{-3}$ in this redshift regime, for the 
TABLE 4

Schechter Function Best-Fit Parameters

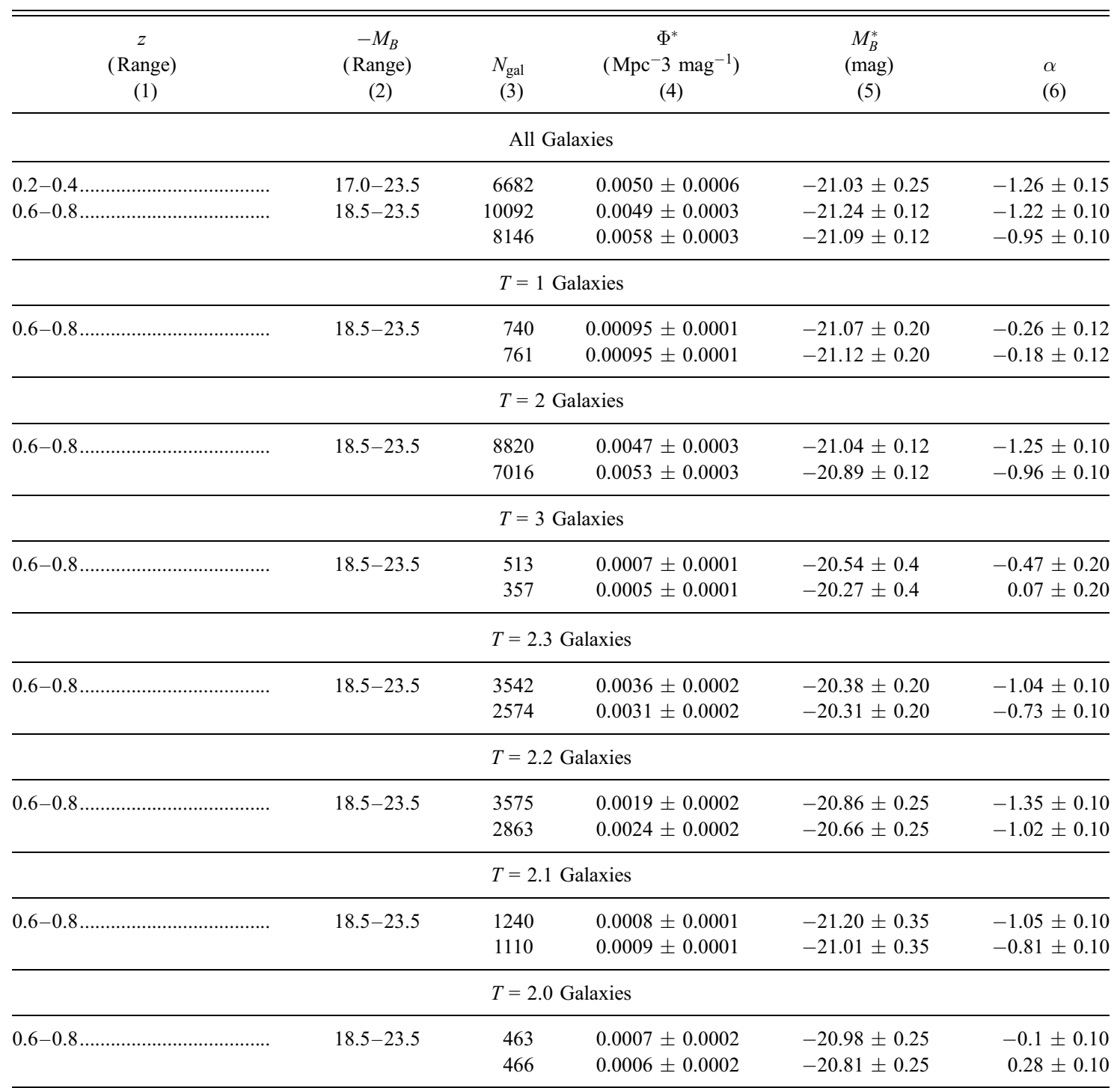

Notes.- The columns are (1) redshift range, (2) $B$-band absolute magnitude range, (3) total number of galaxies considered in the fit, (4) Schechter function normalization and error, (5) $M_{B}^{*}$ and error, and (6) faint-end slope of the Schechter function. In the $z=0.7$ bin, two values are provided for each parameter, reflecting the two different estimates for the photometric redshifts, derived with (upper values) and without (lower values) corrections to the photometric catalogs (see text).

COMBO17 survey. ${ }^{17}$ The overdensity in the COSMOS field at $0.2<z<0.4$ is confirmed by the spectroscopic (Lilly et al. 2007) and photometric (Feldmann et al. 2006) redshift distributions. Similarly, a large overdensity at $z=0.75$ is present in the COSMOS field (Scoville et al. 2007b), which is also detected by XMMNewton (Hasinger et al. 2007).

Figure 12 shows that the LF of the global galaxy population does not change significantly as a function of redshift. Qualitatively, the behavior of the global LF can be described by a brightening of the global galaxy population from redshift $z=0.3$ to 0.9 , with the volume density of the entire galaxy population staying basically constant from redshift $z=0.5$ to 0.9 . The type 2 disk galaxy population follows closely the behavior of the global sample as a function of redshift and can be described with a

17 The COMBO17 survey covers a total area of $0.78 \mathrm{deg}^{2}$, in three fields. The measured $\Phi_{*}$ in the different fields ranges from $13.03 \times 10^{-4}$ to $21.17 \times 10^{-4} \mathrm{Mpc}^{-3}$ (Wolf et al. 2003) rather constant number density and a brightening of the stellar populations.

In Figure 14 we show the corrected LFs of $T=2$ disk galaxies split by their bulgeness parameter. As in Figure 12, different columns show the results for different redshift bins from $0.2<z \leq$ 0.4 to $0.8<z \leq 1$. The LFs of different types of disk galaxies are plotted in different rows, from the bulgeless $T=2.3$ disks in the top row to the bulge-dominated $T=2.0$ disk galaxies in the bottom row. In the lowest redshift bin, as the contribution of the bulge component increases (i.e., moving from top to bottom in Fig. 14), the faint end of the LF becomes flatter, and the LF becomes more similar to that of the type 1 galaxies. At low redshifts, the bulge-dominated disk galaxies contribute most of the light at magnitudes brighter than $M_{B}=-21.5$, while their contribution is strongly reduced at magnitudes fainter than $M_{B}=-19.0$. The LF of $T=2.0$ bulge-dominated galaxies is very similar in both shape and normalization to the LF of the $T=1$ early-type galaxies. 


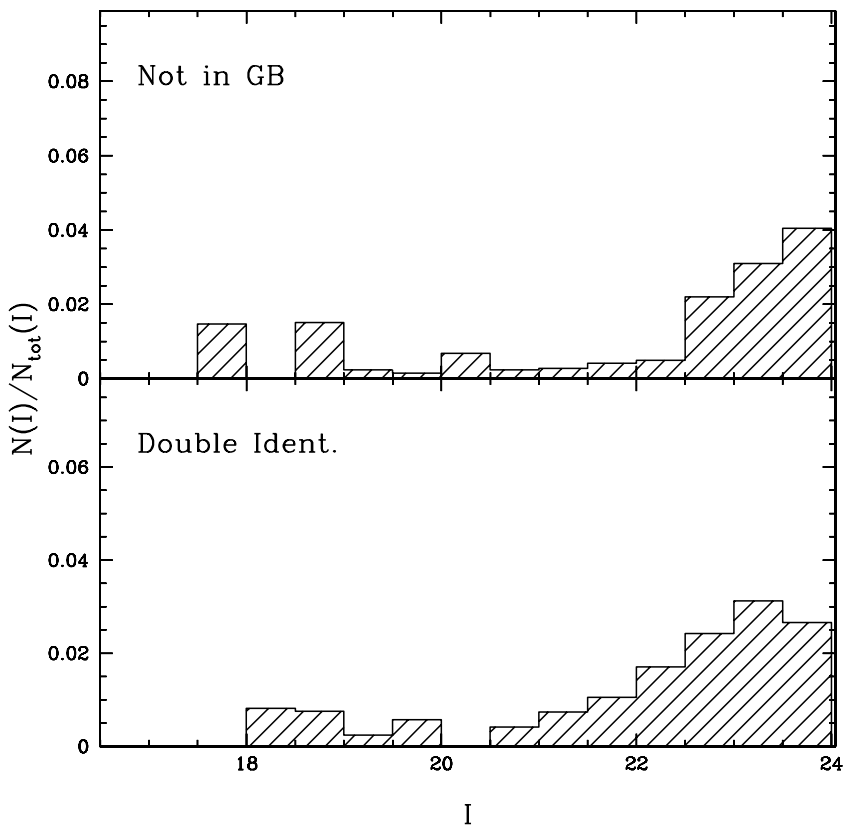

FIG. 11.- The $I$-band magnitude distribution for the ACS galaxies with no detection in the ground-based catalog (top) and for the ACS galaxies whose groundbased match was associated with more than one ACS-detected galaxy. Both distributions are normalized to the magnitude distribution of all galaxies in the sample. The fraction of missed objects stays relatively constant down to $I_{\mathrm{AB}} \sim 22$ and then increases toward fainter magnitudes. [See the electronic edition of the Supplement for a color version of this figure.]

\subsection{Anchoring the COSMOS Evolution to the Local Universe: Comparison with a Complete SDSS-based Sample}

The presence of the $z<0.4$ overdensity in the COSMOS field hampers the interpretation of the evolution of the LFs over the studied redshift range; furthermore, the small volume sampled by the COSMOS survey at redshifts $z<0.2$ is inadequate to constrain the LFs at such late epochs. To correctly compare the COSMOS data with the $z=0$ universe, we used the SDSS-based volumelimited sample of $z=0$ galaxies (appropriately "redshifted" to $z=0.7$, hereafter indicated as $\left.\operatorname{SDSS}_{z=0.7}\right)$ constructed and extensively discussed by Kampczyk et al. (2007); we briefly summarize its properties in Appendix C.

We analyzed the $\mathrm{SDSS}_{z=0.7}$-simulated images following the identical procedure to that we adopted for the COSMOS galaxies: we classified the SDSS galaxies according to their position in the three-dimensional $\mathrm{PC}_{i}$ space and then computed the LFs of the morphologically selected $\mathrm{SDSS}_{z=0.7}$ sample, as described in $\S 5.1$. This $\mathrm{SDSS}_{z=0.7}$ sample provides the properties that local galaxies would show, at redshift $z=0.7$, assuming no evolution between $z=0.7$ and 0 . We therefore stress that applying the identical classification and LF derivation procedures to both our COSMOS and our SDSS-based samples guarantees that any systematics present in the former is also present in the latter; thus, the comparison between the two samples allows us to discover evolutionary trends that are unaffected by such systematics.

The comparison of the COSMOS $0.6<z \leq 0.8 \mathrm{LFs}$ (stars) with the $\operatorname{SDSS}_{z=0.7}$ samples (dash-dotted curves) is shown in

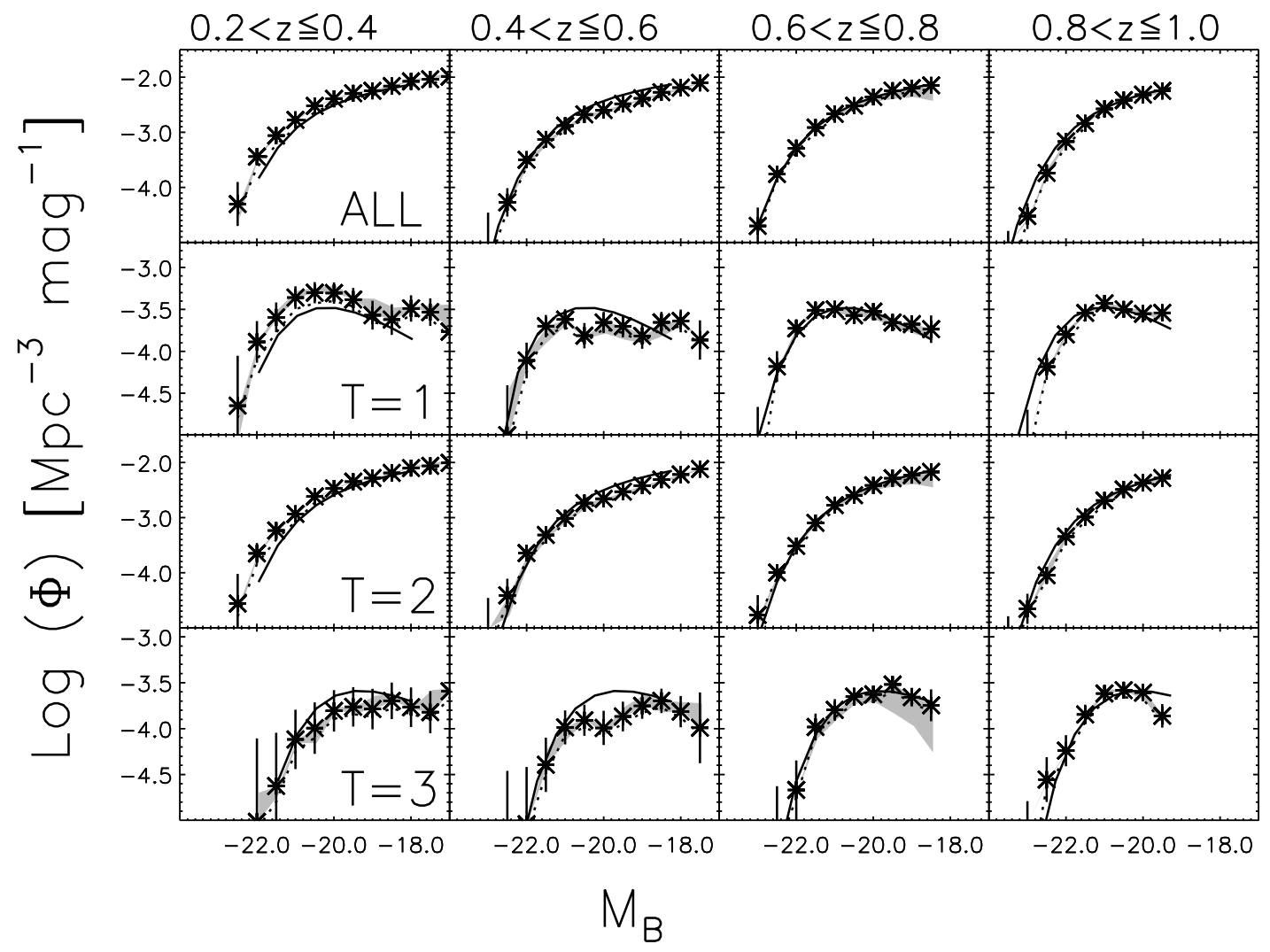

FIG. 12.-The $1 / V_{\max }$ corrected (stars) and uncorrected (dotted lines) LFs derived using the ZEBRA maximum likelihood photometric redshifts for the global sample (top), for $T=1$ early-type galaxies (second row), for $T=2$ disk galaxies (third row), and for $T=3$ irregular galaxies (bottom). In each row, we show the LFs in four redshift bins: $0.2<$ $z \leq 0.4$ (first column), $0.4<z \leq 0.6$ (second column), $0.6<z \leq 0.8$ (third column), and $0.8<z \leq 1.0$ (last column). Error bars in each luminosity bin take into account Poissonian errors only. In each redshift bin LFs are plotted down to the $B$ band at which the sample is complete, regardless of the color of the galaxy. Solid curves show the best-fit Schechter function to the COSMOS LFs in the redshift interval $0.6<z \leq 0.8$, (de)brightened in each redshift range by $\sim 1.3 z$ mag (see text). The stars show the LFs obtained using the ZEBRA photometric redshifts derived after correcting the photometric catalog for detected systematic calibration errors; gray shaded regions show the uncertainty in the LFs due to the use of photometric redshifts derived without applying any correction to the photometric catalog. [See the electronic edition of the Supplement for a color version of this figure.] 


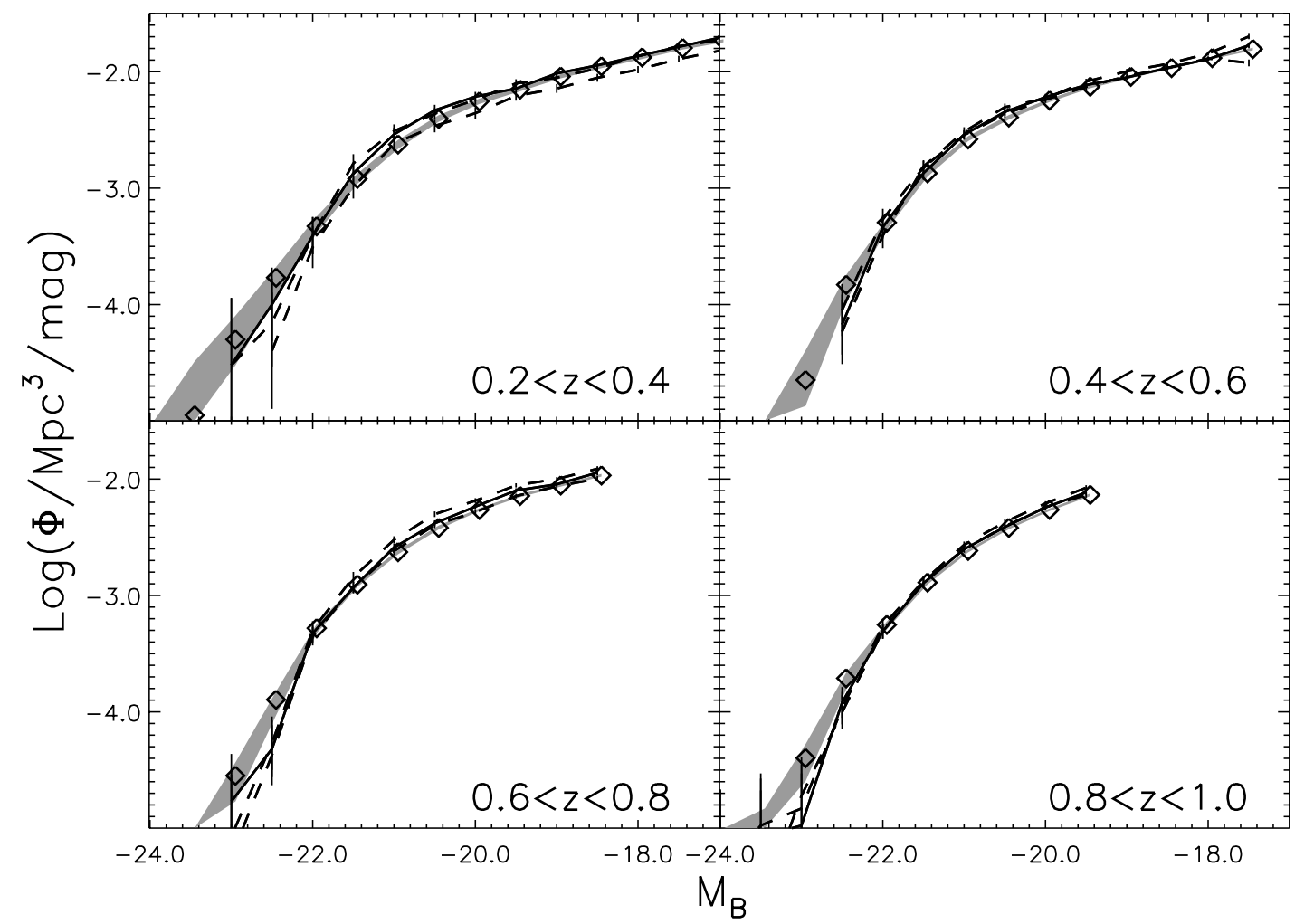

Fig. 13.- Results of the simulations performed to assess the impact of the photometric redshift errors in the calculation of the LF, in four redshift bins, as indicated in each panel. Diamonds represent the median volume density computed from the 100 mock realizations; the associated gray area indicates the 16 th to 84 th percentiles of the distribution in each magnitude bin. Points are shifted by $0.05 \mathrm{mag}$ from the center of the magnitude bin for presentation purposes. The solid line with error bars represents the LF of the original mock catalog used to generate the 100 mock data sets. For comparison, we also show the LFs derived using two independent mock catalogs (dashed lines). These LFs show that cosmic variance in the lowest redshift bin dominates the uncertainty budget in the calculation of the LF errors. [See the electronic edition of the Supplement for a color version of this figure.]

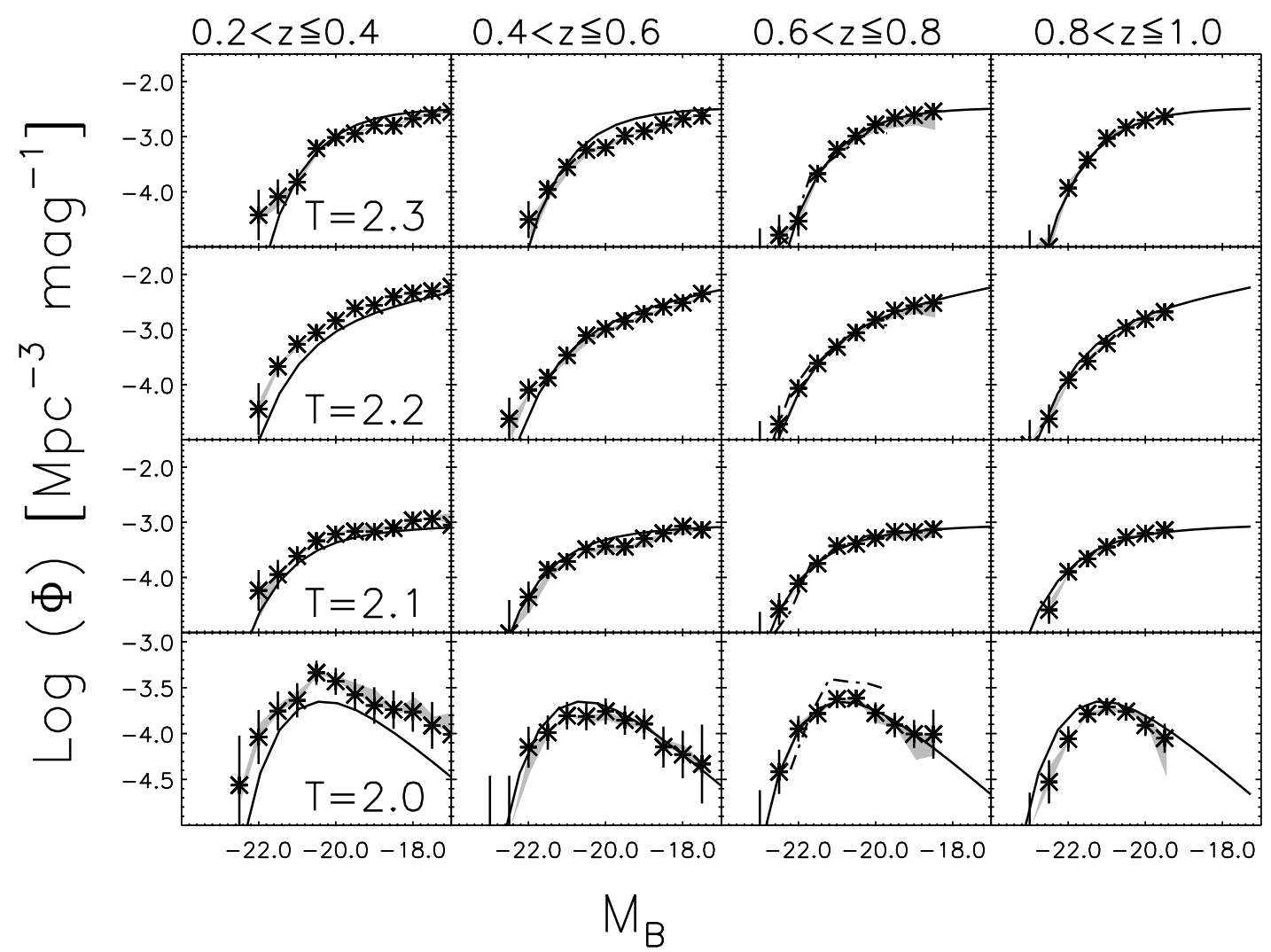

Fig. 14. - Corrected $1 / V_{\max }$ LF computed for $T=2$ galaxies, split by their bulgeness parameter, from $T=2.3$ bulgeless disks (top) to $T=2.0$ bulge-dominated galaxies (bottom). Dash-dotted curves in the redshift bin $0.6<z<0.8$ show the LFs derived for the SDSS ${ }_{z=0.7}$ comparison sample. The solid curves show the best-fit Schechter function to the COSMOS LFs in the redshift interval $0.6 \leq z \leq 0.8$, brightened in each redshift range by $\sim 1.3 z$ mag (see text). Gray shaded regions are as in Fig. 12. [See the electronic edition of the Supplement for a color version of this figure.] 


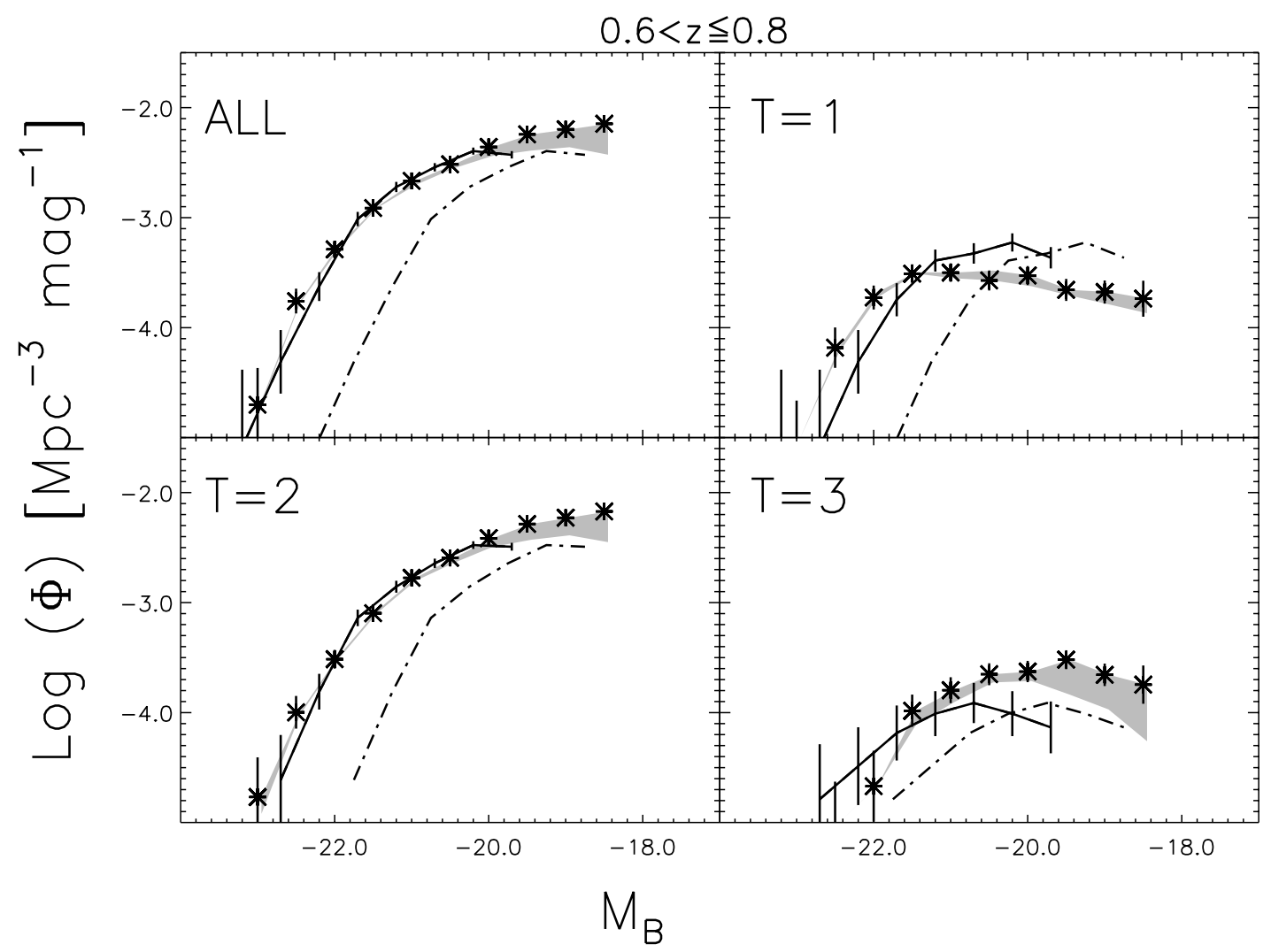

FIG. 15.-Comparison between the corrected COSMOS LFs (stars) and the (redshifted) $\operatorname{SDSS}_{z=0.7}$ LFs, representing the no-evolution predictions at redshift $z=0.7$ (dash-dotted line). The solid curves shows the $\operatorname{SDSS}_{z=0.7} \mathrm{LFs}$ brightened by $0.95 \mathrm{mag}$ (see text for details). Gray shaded regions are as in Fig. 12. [See the electronic edition of the Supplement for a color version of this figure.]

Figure 15. The comparison shows that pure luminosity evolution could explain the differences between the global local SDSS galaxy population and the global COSMOS population at $z=0.7$. To quantify this statement, we compute the brightening of the global local galaxy population that is required to overlap the global $\mathrm{SDSS}_{z=0.7} \mathrm{LF}$ with the global COSMOS LF at redshift $z=0.7$, which is of order $0.95 \pm 0.1 \mathrm{mag}$. This amount of brightening is applied to the $\operatorname{SDSS}_{z=0.7}$ LFs in the various plots of Figure 15 (dashed curves). The agreement between the brightened $\mathrm{SDSS}_{z=0.7} \mathrm{LF}$ derived for the global galaxy population and the global COSMOS $z=0.7 \mathrm{LF}$ is remarkable. Thus, the evolution of the global galaxy population since $z=0.7$ can be interpreted as due to only a brightening of the stellar populations, with no evolution in the average volume density of galaxies over this time period. This result is consistent with recent findings on the evolution of the global LF derived by Willmer et al. (2006) and also Faber et al. (2005) on the basis of the DEEP2 spectroscopic survey.

The Schechter fits to the total, type 1 , type 2 , and type 3 COSMOS LFs in the $0.6<z \leq 0.8$ redshift bins are reported in Table 4 and also in Figure 12 as solid lines. In particular, the Schechter fits to the $z=0.7$ bin are also plotted in the lower redshift bins, after applying the amount of luminosity evolution that is required to overlap the $0.6<z \leq 0.8$ COSMOS LFs with the local SDSS LF (which corresponds to a $B$-band evolution proportional to $\sim 1.3 z$, as in Fig. 15). Despite the noticeable effect in the lowest redshift bin of the known structure in the central part of the COSMOS field, the shapes of the COSMOS LFs at $z \sim 0.7$ describe well, for all the different morphological populations, the LFs at lower and higher redshifts.
Further inspection of Figure 15 shows, however, qualitatively, some interesting evolutionary trends in the individual morphologically selected galaxy samples. In particular,

1. Not surprisingly (since they dominate the global galaxy population), the evolution of the LF of the type 2 disk galaxy population is very similar to that observed in the global population: the LF of disk galaxies is consistent with a pure luminosity evolution of $0.95 \mathrm{mag}$ up to $z=0.7$, with a constant average volume density.

2. Within the errors, the bright end $\left(M_{B}<-21.5\right)$ of the LF of morphologically classified Type 3 irregular galaxies at $z=$ 0.7 is also consistent with that of the local SDSS irregular galaxies (brightened by a similar amount as above). At fainter luminosities, $M_{B}>-20.5$, we detect, however, an excess of a factor of order $\sim 3$ in the number density of faint irregular galaxies at $z \sim 0.7$ relative to the local universe.

3. At bright magnitudes $M_{B}<-21.5$, there is also a good agreement between the LF of $z=0.7$ COSMOS type 1 early-type galaxies and the LF of similarly classified SDSS galaxies brightened by $0.95 \mathrm{mag}$. This evolution is similar to that expected theoretically from the passive evolution of a coeval stellar population formed well before $z \sim 0.7$ and also to the evolution that is observed from studies of the surface brightnesses of early-type galaxies (Rigler \& Lilly 1994; Schade et al. 1999; McIntosh et al. 2005; Holden et al. 2005). This result supports a scenario where most of the massive early-type galaxies are in place at $z=0.7$, with the caveat that we cannot be sure that all of the $z \sim 0.7$ population are actually the progenitors of early-type galaxies (e.g., not all of the $T=1$ galaxies may be consistent with evolving 
onto the Kormendy relation at $z=0$, [Ferreras et al. 2005]; we investigate this issue further in Scarlata et al. [2007]). At fainter magnitudes, $M_{B}>-20.5$, however, there is a drop of a factor of $\sim 2$ between $z=0$ and 0.7 in the number density of morphologically classified early-type galaxies. This result is potentially in contrast with a significant contribution of elliptical-elliptical mergers to building the most massive early-type galaxies at relatively recent epochs and favors a downsizing scenario for earlytype galaxy formation, in which the most massive elliptical galaxies form and dynamically relax at earlier epochs than the lower mass elliptical galaxies. This is also suggested by some studies of elliptical galaxies in the local universe (e.g., Carollo et al. 1993; Thomas \& Davies 2006) and at high redshifts (e.g., Treu et al. 2005; Daddi et al. 2005).

The ZEST misclassification at the faintest magnitudes discussed in $\S 3.2$ is not responsible for the observed deficit of type 1 earlytype galaxies and increase in the number density of type 3 galaxies, since (1) at most $\sim 30 \%$ of type 1 galaxies could be misclassified as type 2 or 3 galaxies; this fraction is too small to explain (a) the missing factor of 2 of type 1 galaxies at redshift $z=0.7$ and (b) the factor of 3 excess in type 3 galaxies observed in the same redshift bin. (2) The $\mathrm{SDSS}_{z=0.7}$ galaxy sample accurately simulates the COSMOS ACS images. Therefore, the same fraction of COSMOS and $\mathrm{SDSS}_{z=0.7}$ galaxies would be misclassified by ZEST due to low $\mathrm{S} / \mathrm{N}$. Thus, the relative comparison of the two samples is not affected by misclassification due to low $\mathrm{S} / \mathrm{N}$.

We postpone to future publications more sophisticated analyses of the LFs of COSMOS galaxy samples that are based on the structural/morphological classification obtained with ZEST.

\section{SUMMARY}

The Zurich Estimator of Structural Types (ZEST) uses the Sérsic index $n$ of the fits to the galaxy surface brightness distributions and five basic nonparametric diagnostics to quantify the properties of galaxy structure. The novelty of ZEST is to use a principal component analysis to retain the full information provided by the entire set of diagnostics while reducing to three the dimensionality of the structural parameter space (of axes $\mathrm{PC}_{1}, \mathrm{PC}_{2}$, and $\mathrm{PC}_{3}$ ). The ZEST scheme morphologically classifies galaxies into three main types (early type galaxies, disk galaxies, and irregular galaxies), assigns a bulgeness parameter related to B/D (quantized in four bins) to disk galaxies, and ranks galaxies according to the elongation, irregularity, and clumpiness of their light distribution. It is most important to stress that, in contrast with other approaches to morphological classifications that use only two or three of the basic structural parameters that enter the ZEST PCA, the ZEST $\mathrm{PC}_{1}-\mathrm{PC}_{2}-\mathrm{PC}_{3}$ classification scheme substantially reduces the contamination in each morphological class from different galaxy types. We have used a sample of $\sim 56,000 I_{\mathrm{AB}} \leq$ 24 galaxies in the central $0.74 \mathrm{deg}^{2}$ of the COSMOS field to calibrate the ZEST classification grid and, so far, ascertained the robustness of the ZEST classification down to the faintest magnitudes of our sample; work to stabilize the results down to even fainter magnitudes is in progress.

As a first application, we use our ZEST-classified sample of COSMOS galaxies to study the evolution since $z=1$ of the luminosity functions (LFs) of the early-type, disk (of different bulgeness parameter) and irregular galaxies. Our analysis shows that the average volume density of disk galaxies remains constant through the studied redshift range, although the stellar populations of these systems are brightened at earlier epochs. In contrast, only the bright, $M_{B}<-21.5$ end of both the irregular and the early-type galaxies remains roughly consistent with the brightened LF of local galaxies. At faint magnitudes, both morphologically classified irregular and early-type galaxies show substantial evolutionary effects from $z=0$ to 0.7 and above. In particular, there is an excess of a factor of $\sim 3$ of faint morphologically classified irregular galaxies, and a deficit of a factor of $\sim 2$ of faint $\left(M_{B}>-20.5\right)$ morphologically classified earlytype galaxies at $z \sim 0.7$ relative to the local universe.

The ZEST classification of our sample of 56,000 COSMOS galaxies has already been used to study the number density evolution of disks of different sizes (Sargent et al. 2007), the LF evolution of plausible progenitors of elliptical galaxies (Scarlata et al. 2007), and the evolution of the merger rate out to $z \sim 1$ (Kampczyk et al. 2007).

The HST COSMOS Treasury program was supported through NASA grant HST-GO-09822. We gratefully acknowledge the contributions of the entire COSMOS collaboration. More information on the COSMOS survey is available at http://cosmos.astro .caltech.edu. We thank the NASA IPAC/IRSA staff for providing online archive and server capabilities for the COSMOS data sets. C. S. acknowledges support from the Swiss National Science Foundation.

Facilities: HST (ACS)

\section{APPENDIX A}

\section{DETAILS ON THE COSMOS GALAXIES}

\section{A1. THE SAMPLE}

A detailed discussion of the ACS data processing is given in Koekemoer et al. (2007); the description of the generation of the ACS catalog that we use in this paper is given in Leauthaud et al. (2007). Briefly, the ACS catalog was produced by applying SExtractor (ver. 2.4.3; Bertin \& Arnouts 1996) to the reduced ACS images. Edge regions in each image were removed with no loss of data, since the overlap between neighboring ACS tiles was larger than the excluded edge regions. Two SExtractor runs were performed in order to avoid both blending of multiple independent sources and deblending of large galaxies, i.e., a "cold" run with a configuration optimized for the detection of large, bright objects and a "hot" run with a configuration optimized for faint, small objects. The two resulting samples were then merged together to produce a final catalog by retaining all the cold detections plus the hot detections that fell outside the SExtractor segmentation map of any galaxy detected in the cold run.

We visually inspected the catalog to remove any residual overdeblending of large galaxies and also false detections. Specifically, we cross-correlated the ACS catalog with the COSMOS CFHT $I$-band catalog down to $I=24.5$ using the celestial coordinates of the 


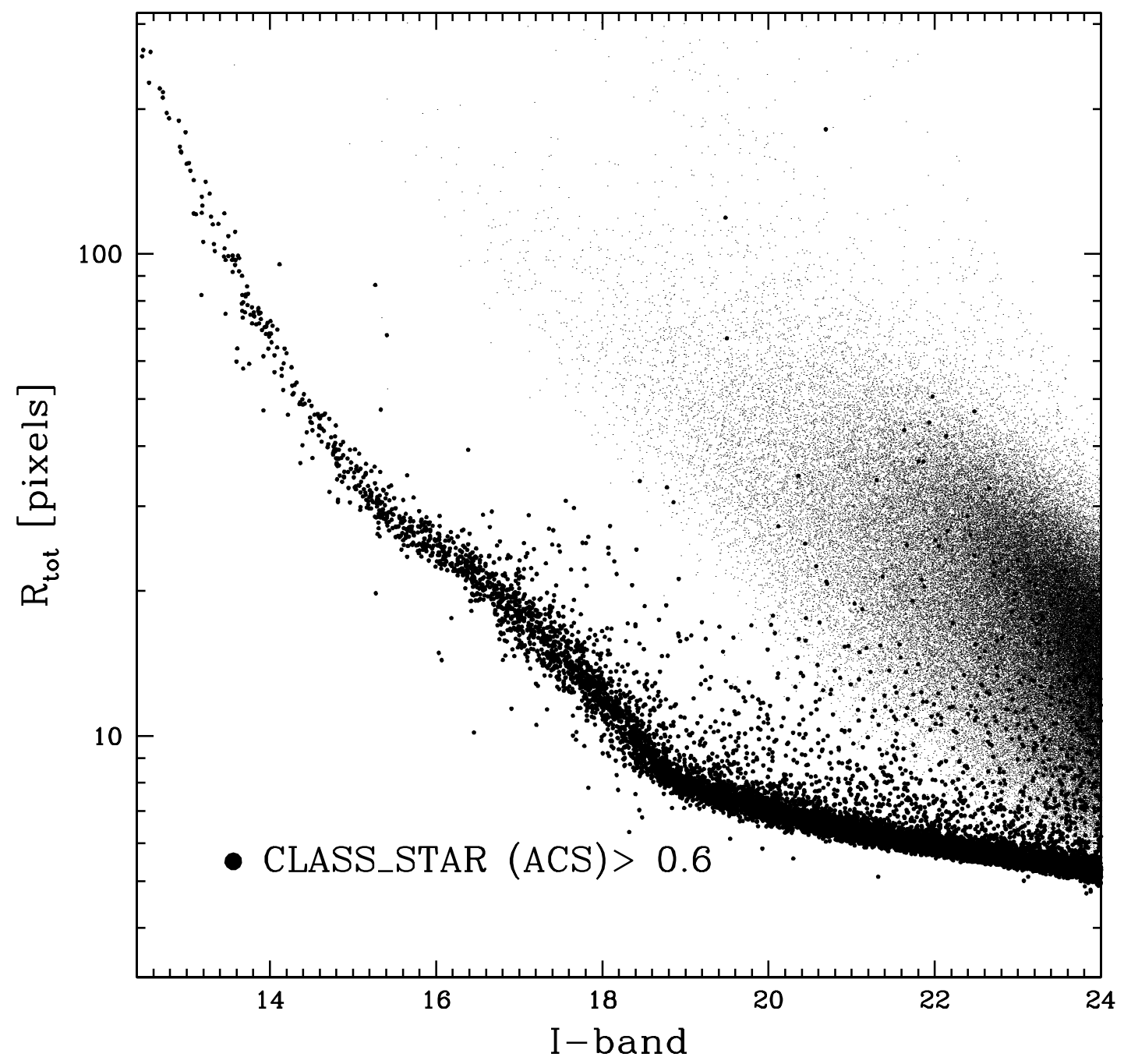

FIG. 16. - Total radius $R_{\text {tot }}$ vs. $I$-band magnitude for all COSMOS galaxies with $I_{\mathrm{AB}} \leq 24$. All objects with CLASS $>0.6$ are identified as filled circles. Down to $I_{\mathrm{AB}}=24$, stars form a tight sequence in this plane, which can be identified using the SExtractor CLASS_STAR parameter. [See the electronic edition of the Supplement for a color version of this figure.]

objects ( $5 \sigma$ magnitude limit of $I_{\mathrm{CFHT}}=24.9$; Capak et al. 2007) ${ }^{18}$ and flagged for visual inspection the ACS-detected objects that (1) had no match in the available ground-based COSMOS catalog; (2) were detected in the ground-based catalog, but had a $>0.5 \mathrm{mag}$ difference between the ground-based and HST I magnitude estimates; and (3) were linked with a CFHT source that had already been associated with another ACS source. About $4.5 \%$ of the entries in the original ACS-based catalog were removed after the visual inspection.

Stars were then removed from the ACS-based catalog using the SExtractor CLASS_STAR parameter, in particular, sources with CLASS $>0.6$. In Figure 16 we show the SExtractor estimate of the radius $R_{\text {tot }}$ including $100 \%$ of the light of an object as a function of the SExtractor ACS I-band magnitude. Objects with CLASS $>0.6$ are identified as filled circles. These form a tight, wellidentifiable sequence at all magnitudes. The final, cleaned, ACS-based catalog that we classify in this paper includes $\sim 55,651$ galaxies down to $I_{\mathrm{AB}}=24$.

\section{A2. DATA ANALYSIS}

The steps of data analysis that we performed on the 56,000 COSMOS galaxies of our sample are summarized below.

1. For each ACS-detected galaxy, we removed in the ACS images the contamination from nearby objects that could affect the computation of the nonparametric coefficients. To create a "cleaned" version of each galaxy image, a stamp centered around the ACSdetected galaxy was extracted from the original ACS tile, with size equal to $3 R_{\text {tot }}$. SExtractor was used to identify the individual sources in the stamp, and the pixels belonging to contaminant galaxies were replaced by background noise that matched the properties of the noise in the original ACS image.

\footnotetext{
${ }^{18}$ For each ACS source we searched for CFHT-detected galaxies within a circle of 0.6 " radius. If more than one CFHT source was found within this radius, the closest CFHT galaxy was assigned to the ACS source as its ground-based counterpart.
} 


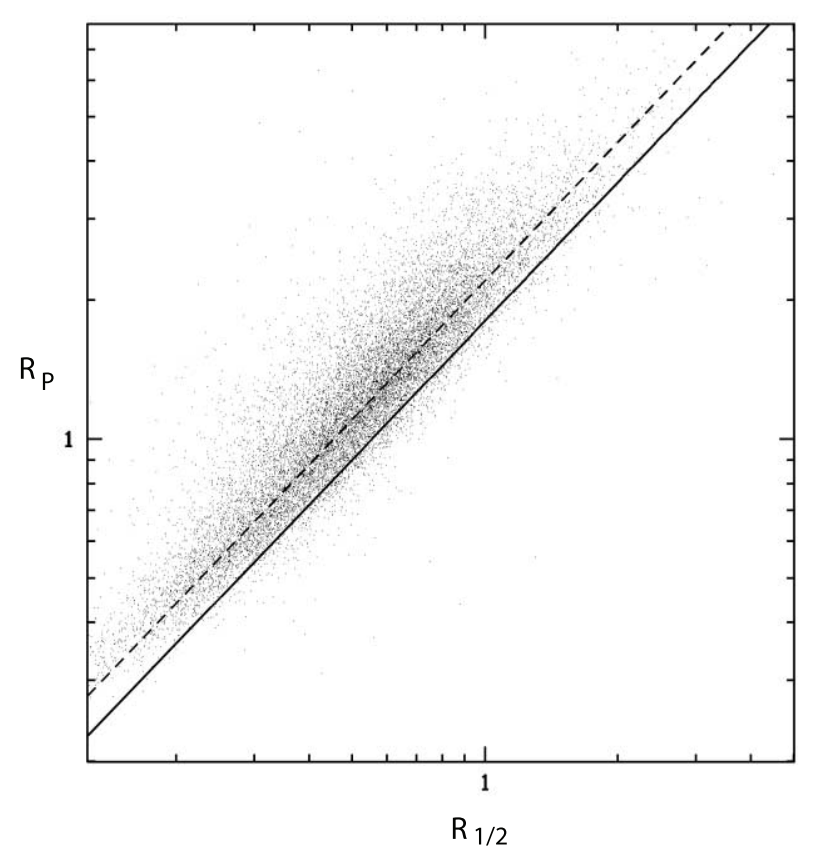

FIG. 17. - Comparison between the Petrosian radii of $I_{\mathrm{AB}} \leq 24$ COSMOS galaxies, as derived in our analysis, and the half-light radii derived by Sargent et al. (2007) for the $I_{\mathrm{AB}} \leq 22.5$ subsample (using GIM2D fits to the galaxy surface brightness distribution). The solid and dashed lines represent the relation between $R_{\mathrm{P}}$ and $R_{1 / 2}$ expected for a Sérsic profile with $n=1$ and 2, respectively. [See the electronic edition of the Supplement for a color version of this figure.]

2. The Petrosian radius (Petrosian 1976; $R_{\mathrm{P}}$ ) of each galaxy was calculated from the surface brightness profile measured from its "cleaned" stamp. ${ }^{19}$ The surface brightness profiles were derived using elliptical annuli centered on the galaxies, in order to minimize the contribution by background pixels. The position angle and ellipticity of the annuli were kept fixed at all radii to the values measured from the image moments (Bertin \& Arnouts 1996). The annuli were uniformly spaced in $\log (R)$ to increase the $\mathrm{S} / \mathrm{N}$ in the external region of galaxies. Each galaxy profile was sampled by at least 10 points. We calculated $R_{\mathrm{P}}$ for all galaxies with a HWHM (as estimated by SEXtractor) larger than 1.5 pixels, so as to ensure that enough pixels were available for the measurement of the parameters. This cut excludes $\sim 0.2 \%$ of the entire sample of COSMOS galaxies under consideration. In Figure 17 we show, for the sample of $I_{\mathrm{AB}} \leq 22.5$ galaxies analyzed by Sargent et al. (2007), the comparison between the Petrosian radius derived in our analysis and the half-light radius derived by Sargent and collaborators from GIM2D fits (Simard 1998) to the galaxy surface brightness distributions. The solid and dashed lines represent the relation between $R_{\mathrm{P}}$ and $R_{1 / 2}$ expected for a Sérsic profile with $n=1$ and 2, respectively. The very good correlation between the two measured quantities indicates that our Petrosian radii reliably reproduce the galaxy sizes and offer the advantage of expanding the COSMOS galaxy sample with computed robust size measurements down to $I_{\mathrm{AB}}=24$.

3. The pixels that are associated with a galaxy were defined as all the pixels within elliptical apertures of semimajor axis equal to the Petrosian radius of the galaxy. With this definition, the same fraction of light is considered, for any given shape of the surface brightness profile, at different redshifts, therefore allowing one to consistently compare measurements of galaxy populations at different epochs.

In Figure 18 we illustrate with a few examples the procedure outlined so far. In particular, we show four COSMOS galaxies with increasing magnitudes, from $I_{\mathrm{AB}}=18.8($ top $)$ to $I_{\mathrm{AB}}=22.3$ (bottom). For each galaxy we show the original stamp ( first column), the cleaned stamp (second column), the segmentation map (third column), and in the last column, the surface brightness profile (top) and the ratio $\eta=\mu(R) /\langle\mu(<R)\rangle$ (bottom) as a function of radius $R$ in arcseconds ( filled circles). The solid horizontal line in the $\eta-R$ plots represents $\eta=0.2$. For the galaxy in the bottom row, $\eta$ decreases to a minimum value that is, however, larger than $\eta=0.2$ and then increases again at larger radii. This is due to the contamination from a nearby source, still visible even in the cleaned stamp, which adds flux in the outermost isophotes (as easily seen in the $\mu$ radial profile). To obtain reliable Petrosian radii, we corrected these cases by fitting a linear relation to the $\mu-R$ profile in the outer radial bins (dashed line) and then subtracting a constant value from the surface brightness profile. The open circles in the $\eta-R$ plot of the bottom-row galaxy in Figure 18 show the $\eta$-profile obtained after the application of this procedure and demonstrate that we recover robust values of $R_{\mathrm{P}}$ even in these potentially troublesome cases.

\section{A3. PHOTOMETRIC REDSHIFTS FOR COSMOS GALAXIES}

Ancillary ground-based photometry in several passbands is available for the COSMOS field from different facilities (see summary in Scoville et al. 2007a). This allows the estimate of accurate photometric redshifts for the COSMOS galaxies. Due to the differences in adopted catalogs, and the intrinsic uncertainties in photometric redshift estimates, the COSMOS collaboration has decided to adopt several approaches to determine photometric redshifts for COSMOS galaxies. Mobasher et al. (2007) present a comparison among several estimates for the COSMOS photometric redshift, obtained by different groups within the COSMOS team.

19 The radius $R_{\mathrm{P}}$ is defined as that where the ratio $\eta=\mu(R) /\langle\mu(<R)\rangle$ between the surface brightness at that radius and the surface brightness averaged within that radius is equal to a given number. For a surface brightness distribution described by a de Vaucouleurs or an exponential profile, a value of $\eta=0.2$ is reached at $R_{\mathrm{P}} \sim 1.8 R_{1 / 2}$ and $R_{\mathrm{P}} \sim 2.2 R_{1 / 2}$, respectively (with $R_{1 / 2}$ the half-light or "effective" radius of the galaxy; Graham \& Driver 2005). 

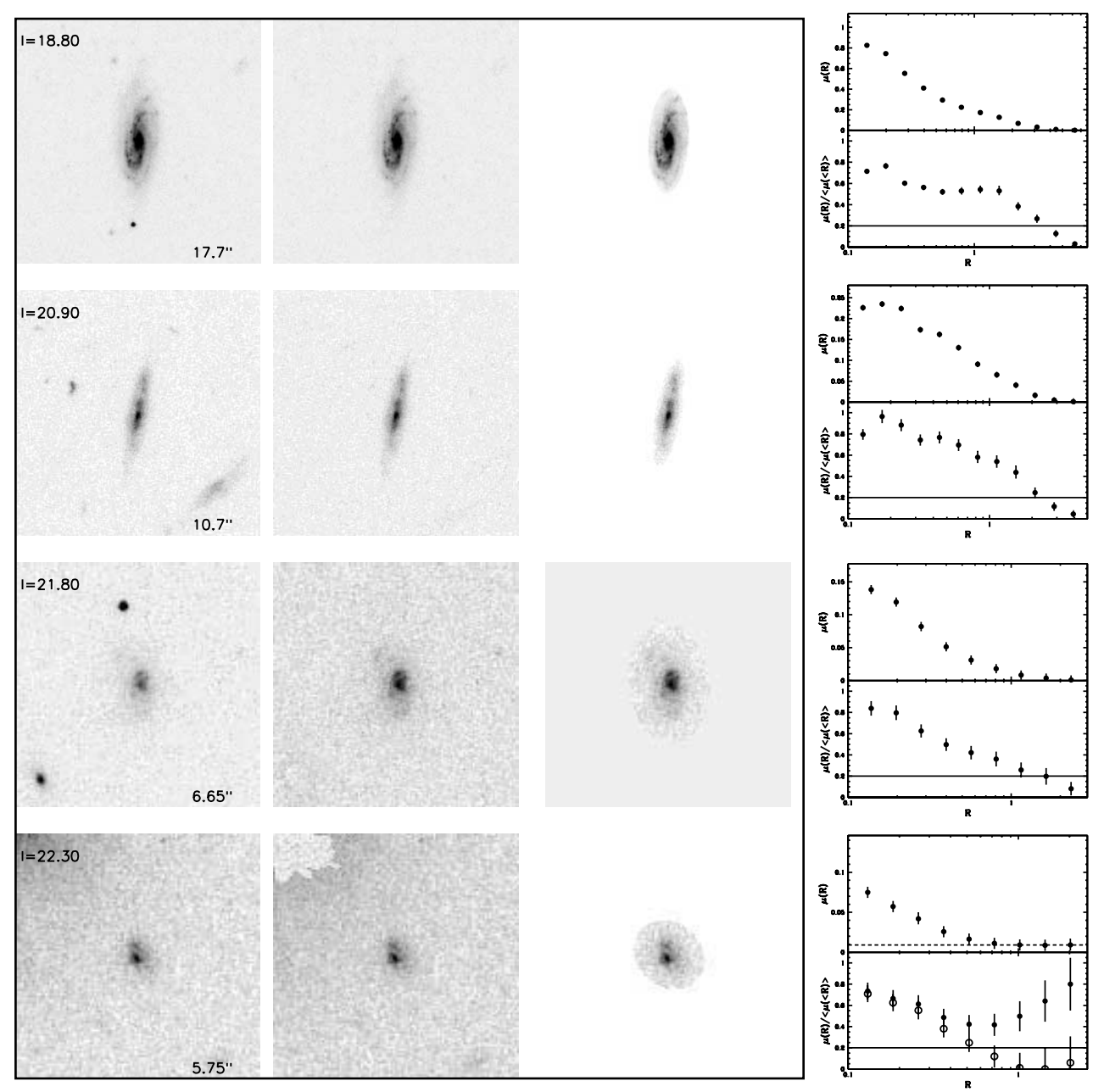

FIG. 18. - Examples of the steps performed to compute the Petrosian radius $R_{\mathrm{P}}$. In particular, we show four galaxies with increasing magnitudes, from $I=18.80$ (top) to $I=22.3$ (bottom). For each galaxy we show the original stamp ( first column), the cleaned stamp (second column), and the segmentation map (third column). In the last column we show the surface brightness profile $($ top $)$ and the ratio $\eta=\mu(R) /\langle\mu(<R)\rangle$ (bottom) as a function of $R$ in arcseconds ( filled circles). The solid horizontal line in the $\eta-R$ plots shows $\eta=0.2$. In the last example, $\eta$ decreases to a minimum value larger than $\eta=0.2$ and then increases again. This behavior is due to contamination to the external isophotes from light coming from the wings of the nearby source, still visible in the cleaned stamp, despite the attempt to remove it before proceeding with the analysis. This light causes the surface brightness profile to flatten at large radii, as seen in the $\mu-R$ diagram. To correct for this effect, we subtract the residual light contribution from the nearby source. The correction is derived by fitting a linear relation (shown as dashed lines in the $\mu-R$ plots) to the external part of the light profile in the $\mu-R$ plot and then subtracting a constant value from the surface brightness profile. Open circles in the $\eta-R$ plots show the $\eta$-profiles obtained after this correction is applied. [See the electronic edition of the Supplement for a color version of this figure.]

In our work, we adopt the photometric redshifts for the COSMOS galaxies of our ACS-selected $I_{\mathrm{AB}} \leq 24$ galaxy sample by Feldmann et al. (2006). These are derived with ZEBRA. ZEBRA produces two separate estimates for the photometric redshifts of individual galaxies: a maximum likelihood estimate and a two-dimensional Bayesian estimate. In this paper we use the maximum likelihood photometric redshifts. These were derived using: (1) Subaru photometry in six broadband filters: $B, V, \mathrm{G}, r^{\prime}, i^{\prime}$, and $z^{\prime}(5 \sigma$ magnitude limit of $\sim 27$ for point sources in all bands; see Taniguchi et al. [2007] for details); (2) $u^{*}$ photometry from the MegaCam at the CFHT ( $5 \sigma$ magnitude limits for point sources of $\left.u^{*}=27.4\right)$; and (3) $K_{s}(2.2 \mu \mathrm{m})$ photometry from the National Optical Astronomy Observatory (NOAO) wide-field infrared imager Flamingos (mounted on the Kitt Peak $4 \mathrm{~m}$ telescope) and the Infrared Side Port Imager (ISPI) at the Cerro Tololo Inter-American Observatory (CTIO) Blanco $4 \mathrm{~m}$ telescope.

The sources in the ground-based catalog were detected from the original (i.e., nonmatched and nonhomogenized PSFs) Subaru $i^{\prime}$ images, since these had, on average, the best seeing condition of $\sim 0.6^{\prime \prime} \mathrm{FWHM}$, allowing for an optimal deblending of close galaxy pairs. Photometric fluxes were instead estimated on PSF-matched images. All magnitudes were calculated within circular apertures of 3.0" diameter. The final ground-based catalog of Capak et al. (2007) includes $>10^{6}$ galaxies down to $i^{\prime}=26$ over the entire COSMOS field.

In deriving the LFs for the COSMOS galaxies, we checked that, in addition to being insensitive to slightly different photometric redshift estimates derived by applying or ignoring small shifts in the calibration of the groundbased photometry (see $\S 5$ ), the results remain unchanged also when using the full ZEBRA Bayesian probability distribution for the photometric redshift estimates. Furthermore, the LFs do not vary when computing them (1) after excluding from the sample all galaxies with ZEBRA $\chi^{2}$ values above the 95th percentile of the $\chi^{2}$ distribution and (2) by including all galaxies in the sample, independent of the $\chi^{2}$ value of their ZEBRA fits.

The ZEBRA photometric redshift estimates of the COSMOS galaxies are less accurate for $I_{\mathrm{AB}}>22.5$ sources not only due to their larger photometric errors, but also due to the lack of photometric redshift calibration at such faint magnitudes. The ongoing spectroscopic follow-up of COSMOS on the ESO VLT, zCOSMOS, will secure spectroscopic redshifts for $>35,000$ COSMOS galaxies (Lilly et al. 2007). So far, however, only about 1500 COSMOS galaxies have VLT spectroscopic redshifts, most of which are in the $0.2<z<1.1$ 


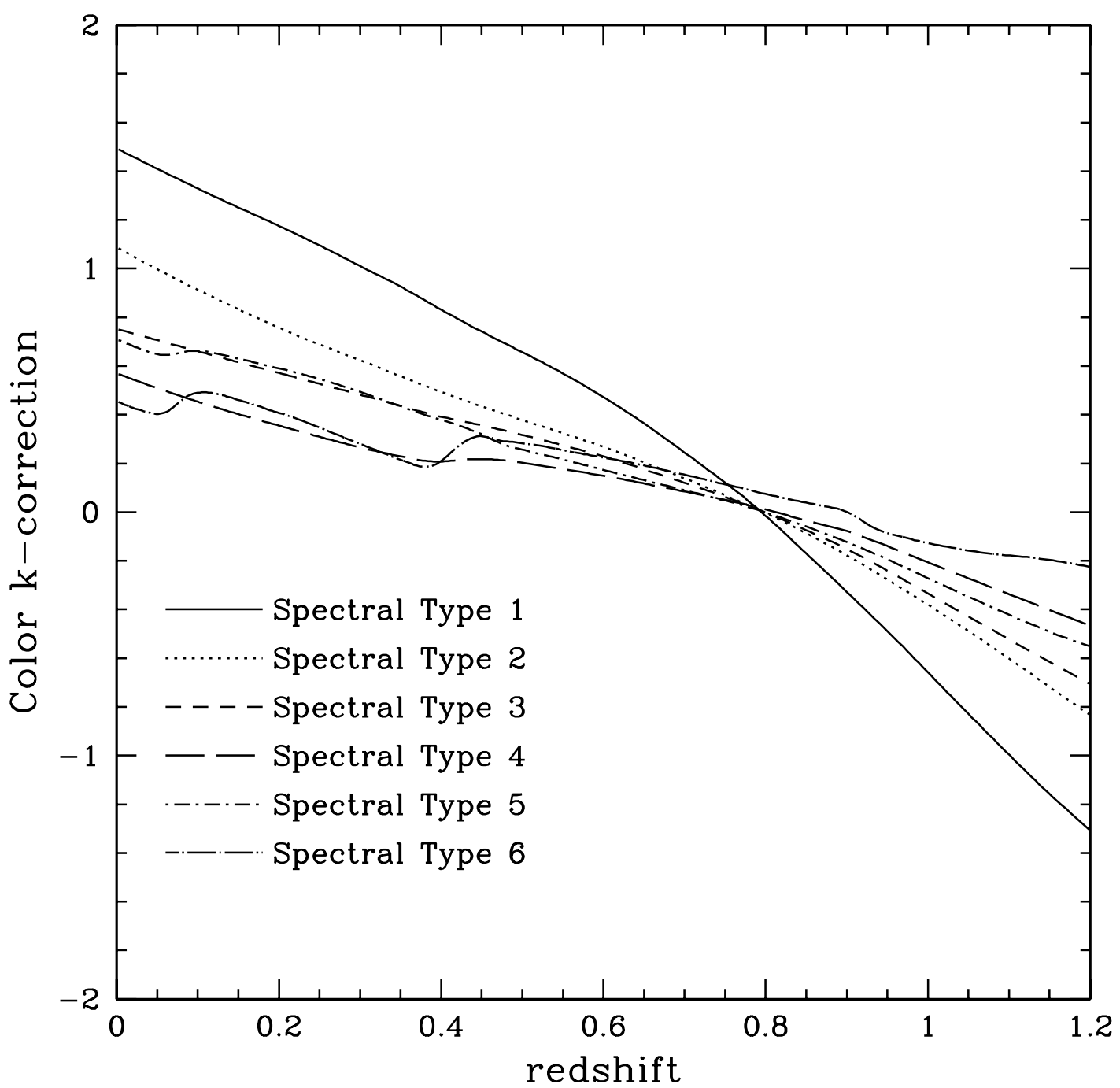

Fig. 19.-Color $k$-correction [defined as $k(z)=B-I_{z}$, see text for details] as a function of redshift for different spectral templates, going from an early-type galaxy template ( $T=1$, solid line) to the starburst galaxy template $(T=6$, long-dash-dotted line). At redshift $z \sim 0.8$ the color $k$-correction is virtually zero for all photometric types, since at this redshift the rest-frame $B$ band coincides with the $I$-band filter from which the $B$-band absolute magnitudes are derived. [See the electronic edition of the Supplement for a color version of this figure.]

redshift range, at magnitudes brighter than $I_{\mathrm{AB}}=22.5$. In order to estimate the accuracy of the ZEBRA photometric redshifts at magnitudes fainter than $I_{\mathrm{AB}}=22.5$, we simulated a faint version of the spectroscopic redshift catalog by dimming the $I_{\mathrm{AB}}<22.5$ galaxies with available spectroscopic redshift down to our magnitude limit of $I_{\mathrm{AB}}=24$. We then recomputed the ZEBRA redshifts for these artificially dimmed sources. The accuracy of the ZEBRA photometric redshifts down to $I_{\mathrm{AB}}=24$ is $\sigma /(1+z) \sim 0.06$.

\section{A4. REST-FRAME QUANTITIES}

Total magnitudes for the COSMOS galaxies in our sample were available in the ACS I band. In order to compute the rest-frame absolute $B$-band total magnitude $\left(M_{B}\right)$, we computed for each COSMOS galaxy the color $B-I_{z}$, where $B$ is the observed magnitude at $\lambda_{B, \text { obs }}=(1+z) \lambda_{B}$ and $I_{z}$ indicates the observed $I$-band magnitude for a galaxy at redshift $z$. The central wavelength of the ACS filter corresponds to the central wavelength of the $B$-band at redshift $z \sim 0.8$; therefore, $B-I_{z=0.8}=0$. Defining the color $k$-correction as $K(z)=B-I_{z}$, the rest-frame absolute magnitude $M_{B}$ can be expressed as

$$
M_{B}=I_{z}-5.0 \log \left(d_{L}(z) / 10 \mathrm{pc}\right)-25.0+K(z)+2.5 \log (1+z)
$$

where $d_{L}$ is the luminosity distance at redshift $z$. We computed the color $B-I_{z}$ using the best-fit SEDs derived for each galaxy as part of the ZEBRA photometric redshift calculation.

In Figure 19 we show $B-I_{z}$ as a function of redshift for our six basic spectral templates going from an early-type galaxy template $(T=1$, solid line) to the starburst galaxy template $(T=6$, long-dash-dotted line). As expected, at redshift $z \sim 0.8$ the $k$-correction is zero for all photometric types.

In the calculation of the LFs, $V_{\max }$ was computed for each galaxy taking into account its ZEBRA photometric type. Specifically, given the galaxy rest-frame $M_{B}$ absolute magnitude, we derived the observed expected $I$-band magnitude as a function of redshift using the 


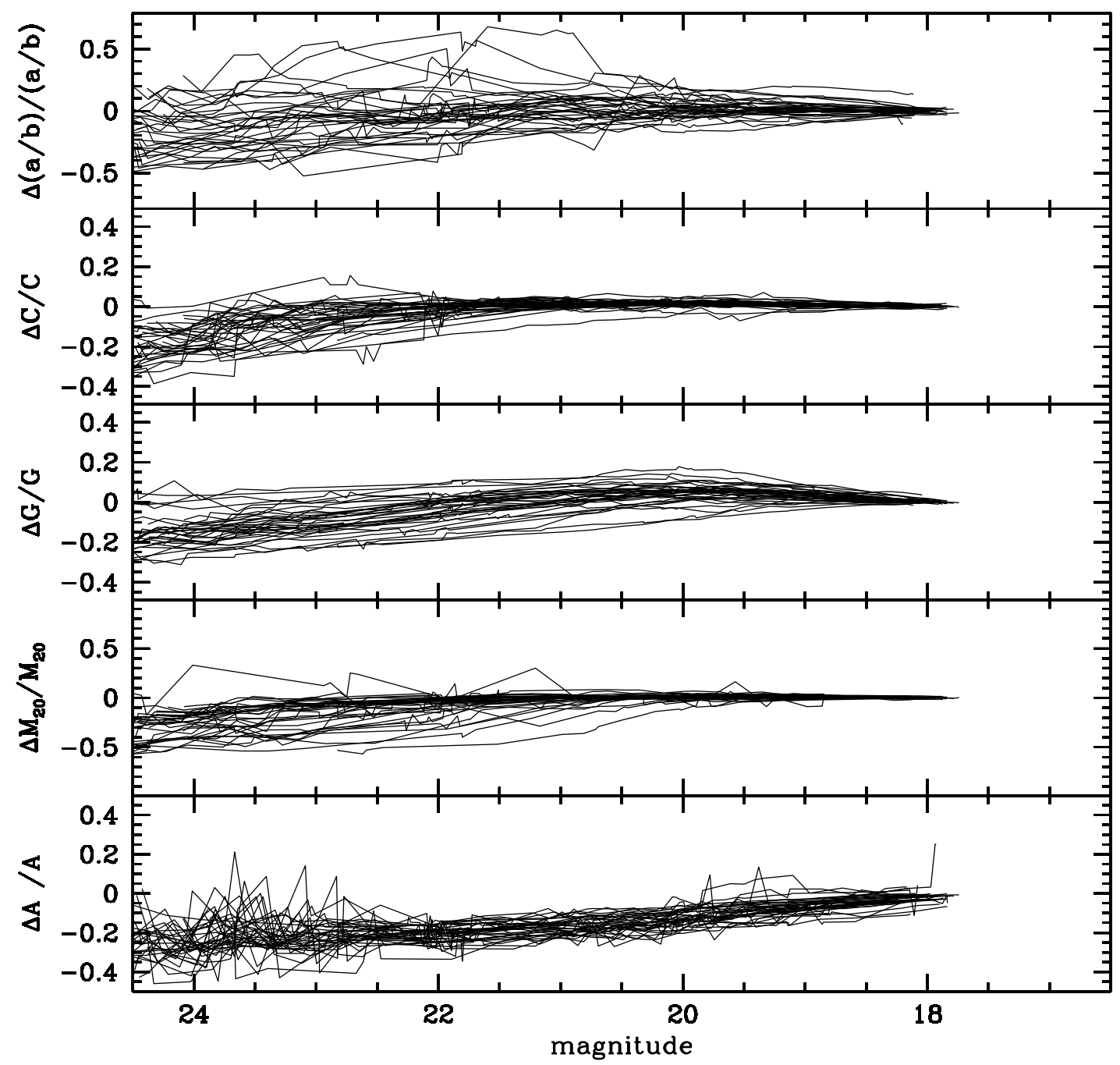

Fig. 20.- Results of the simulations performed by degrading the $\mathrm{S} / \mathrm{N}$ of real galaxies (see text for details). Lines in each panel show the fractional variation of the parameter as a function of magnitude (each line shows a different galaxy). From top to bottom we show ellipticity, concentration, Gini coefficient, $M_{20}$, and asymmetry. Systematic effects are $<20 \%$ over the whole range of observed magnitudes. [See the electronic edition of the Supplement for a color version of this figure.]

galaxy best-fit template derived from the ZEBRA photometric redshift. The $I$-band observed magnitude as a function of redshift is required to find $z_{16}$ and $z_{24}$, which define the redshift range within which the galaxy could still be seen in the survey, and therefore $V_{\max }$.

\section{APPENDIX B}

\section{UNCERTAINTIES AND SYSTEMATICS IN THE MORPHOLOGICAL MEASUREMENTS}

To quantify the errors on the measured parameters for the COSMOS galaxies and to assess systematic trends as a function of galaxy brightness, we performed extensive simulations based on real bright COSMOS galaxies. In particular, we selected 35 bright isolated COSMOS galaxies with magnitude between $I=17.5$ and 18 and, for each of them, we created $40 \mathrm{~S} / \mathrm{N}$-degraded versions of their images. This was done by dividing the original frame by a factor $f$ such that the final magnitude of the object was $2.5 \log (f)$ fainter than the original one. The factor $f$ was chosen so that each galaxy uniformly sampled the magnitude range from 18 to 24 . The scaled images were then added back to an ACS tile, in randomly selected positions. Before adding the rescaled image, the original ACS tile was multiplied by a factor $k$ to preserve the noise properties in the final image. Specifically, with $\sigma_{\text {orig }}$ the background noise in the image containing one of the 35 bright galaxies, the background noise after the rescaling is $\sigma_{\text {orig }} / f$. Adding the rescaled image to the ACS original image increases the noise in the final image to $\sigma_{\text {final }}^{2}=\sigma_{\text {orig }}^{2}+\sigma_{\text {orig }}^{2} / f^{2}$. The factor $k$ is therefore computed by requiring that $\sigma_{\text {final }}^{2}=\sigma_{\text {orig }}^{2}=\sigma_{\text {orig }}^{2} / k^{2}+\sigma_{\text {orig }}^{2} / f^{2}$.

In Figure 20 we show the results of this set of simulations. In particular we show the fractional change of each parameter as a function of the magnitude of the $\mathrm{S} / \mathrm{N}$-degraded galaxy. Different lines connect the variation of the parameters for each galaxy as a function of magnitude.

For all parameters (except $\epsilon$ ) the scatter remains low (at a level of 10\%-20\%) and no strong systematic effects are observed down to $I \sim 22$. At magnitudes $I>22$ the scatter increases, and all parameters are systematically underestimated up to a maximum of $\sim 20 \%$ in the faintest magnitude bin. The behavior of $\epsilon$ shows a different trend, with the scatter starting to increase already at bright magnitudes 
(at a level of $\sim 10 \%$ ), and systematic effects start to be important at $I \sim 23$. The asymmetry $A$ is systematically underestimated at the $10 \%$ level for $I$-band magnitudes of order 21 and degrades down to a $20 \%$ underestimate for $I_{\mathrm{AB}}=24$.

\section{B1. EFFECTS OF THE POINT-SPREAD FUNCTION}

For very compact objects, the structural parameters computed for the COSMOS galaxies from the HST ACS images can be affected by the instrumental PSF (FWHM $\sim 0.1^{\prime \prime}$ ). In order to determine the limiting size/magnitude at which the effects of the PSF become important in our classification algorithm, we performed a set of simulations based on synthetic galaxy images. Specifically, we constructed artificial images of about 2500 galaxies with magnitudes in the range $21 \leq I \leq 24$, surface brightness profiles described either by a de Vaucouleurs (i.e., $n=4)$ or an exponential $(n=1)$ profile, and with half-light radii $R_{1 / 2}$ in the range $0.05^{\prime \prime} \leq R_{1 / 2} \leq 1.0^{\prime \prime}$. These artificial images were convolved with the Tiny Tim (Krist \& Hook 1997) HST PSF and with a PSF constructed from stars present in the COSMOS ACS data. Similar results are obtained with either of these PSFs; in the following, we discuss the results obtained with the TinyTim PSF.

We analyzed and classified, in a similar way as was done for the COSMOS data, both the original and the PSF-convolved artificial galaxies. Comparing the results obtained on the original and on the PSF-convolved images we find that galaxies with the exponential $(n=1)$ surface brightness profile are always classified as type 2 disk galaxies, and no change of class is observed over the entire range of half-light radii and magnitudes explored. For $n=4$ galaxies, we find that all galaxies are classified as type 1 (early-type galaxies) when the analysis is performed on the non-PSF-convolved images. When the convolved images are considered, a fraction of about $15 \%$ of all galaxies with half-light radius larger than $0.17^{\prime \prime}$ changes class from type 1 to type $2 ; 85 \%$ of these systems have $I_{\mathrm{AB}}>23$. For galaxies with an $n=4$ profile, all objects with measured $R_{1 / 2}<0.17^{\prime \prime}$ change class from type 1 to type 2 .

The fraction of objects in our COSMOS sample with measured $R_{1 / 2}<0.17^{\prime \prime}$ is about $4 \%$ down to our magnitude limit of $I_{\mathrm{AB}}=24$. For (the steepest profile galaxies in) this small fraction of the sample, the ZEST morphological classification is therefore likely affected by convolution with the HST ACS PSF.

\section{APPENDIX C}

\section{THE KAMPCZYK ET AL. (2007) SDSS-BASED $z=0$ COMPARISON SAMPLE}

The volume of universe sampled by the Cycle 12 ACS COSMOS data within the $0<z \leq 0.1$ redshift range is only $\sim 5.5 \times 10^{3} \mathrm{Mpc}^{3}$ and includes $<50$ galaxies. Therefore, cosmic variance and small number statistics in this low-redshift bin are inadequate to provide a meaningful $z \sim 0$ calibration for studies of galaxy evolution.

To study the LF evolution down to $z=0$ of the ZEST-classified morphological galaxies, we therefore used the SDSS-based data set of artificial galaxy images constructed and presented by Kampczyk et al. (2007). These simulate how the local universe would be observed in the COSMOS survey at a redshift $z=0.7$. Specifically, the artificial data set is a volume-limited sample of 1813 , $M_{B}<-18.5,0.015<z<0.025$ galaxies extracted from the SDSS-DR4 galaxy catalog. ${ }^{20}$ The absolute $B$-band magnitude $M_{B}$ was derived from the SDSS $g$ magnitude, by applying a $k$-correction computed on the basis of the observed $g-r$ color and redshift of each galaxy.

The SDSS $g$ band at $z=0$ almost perfectly matches the ACS F814W $(I)$ band at $z=0.7$. To simulate how the local galaxies (observed in the SDSS $g$ band) would appear, in the absence of evolution, in the F814W COSMOS ACS images at a redshift $z=0.7$, it is thus only necessary to take into account the different pixel scales and PSFs, and cosmological surface brightness dimming. The $\mathrm{SDSS}_{z=0.7}$-simulated images do not include either surface brightness or size evolution; they were randomly added to the COSMOS ACS images to reproduce the ACS COSMOS noise properties and to simulate galaxy overlapping due to projection effects.

The simulated $\operatorname{SDSS}_{z=0.7}$ galaxies were analyzed with the same procedure used for the real COSMOS data. First, we run SExtractor to detect the sources and to measure their $I$-band magnitudes, position angles, and ellipticities. The same SExtractor configuration parameters that were used to generate the COSMOS catalog (Leauthaud et al. 2007) were adopted for the extraction of the $\operatorname{SDSS}_{z=0.7}$ objects. About $6 \%$ of the $\mathrm{SDSS}_{z=0.7}$ galaxies were not recovered by SExtractor; $0.1 \%$ were instead detected, but with a magnitude fainter than our $I_{\mathrm{AB}}=24$ limit, and were therefore excluded from the $\mathrm{SDSS}_{z=0.7}$ sample. For each simulated galaxy, we then measured the structural parameters described in $\S 2.1$ and applied the ZEST morphological classification described in $\S 2.2$.

The rest-frame $B$-band LF for the constructed $\operatorname{SDSS}_{z=0.7}$ sample is shown in Figure 21 (solid histogram). Being based on a volumelimited, random selection of the local galaxy population, this LF will have the same shape as the global $z=0 B$-band LF. We therefore used, to normalize the volume density, the total number of SDSS galaxies with $M_{B}<-18.5$, which was derived by integrating the $B$-band LF recovered from the $g$-band LF of Blanton et al. (2003). To convert $g$-band to $B$-band magnitudes, we considered two extreme SEDs: a young $(0.1 \mathrm{Gyr})$ single-burst stellar population with solar metallicity, and an old (12 Gyr) single-burst stellar population with the same metallicity. The corresponding transformations are $B=g+0.02$ and $B=g+0.36$. In Figure 21 we show the LFs calculated using the normalization derived adopting the "old" SED (dotted line), the "young" SED (dashed line), and the average of the two (solid line). In discussing the comparison of the COSMOS and $\operatorname{SDSS}_{z=0.7}$ LFs, we adopt the SDSS $B$-band LF derived with the average SED correction.

\footnotetext{
20 The SDSS survey provides homogenous color information and spectroscopic redshifts for more than $10^{6}$ galaxies with magnitude $r<17.77$ (Strauss et al. 2002 ). We expect the Kampczyk et al. (2007) SDSS-based sample to be unbiased toward environmental effects and fully representative of the local galaxy population down to the $M_{B}=-18.5$ magnitude limit, since (1) the photometric +spectroscopic SDSS data are mostly complete down to $r \sim 17.8$ (Strauss et al. 2002), i.e., well below the considered absolute magnitude cut. Specifically, the surface brightness cut applied for the selection of the SDSS spectroscopic targets $\left(\mu<23\right.$ mag arcsec $\left.^{-2}\right)$ and mechanical constraints of the spectrograph (which exclude galaxies in pairs closer than 55") remove less than $1 \%$ and $\sim 6 \%$ of targets brighter than $r=17.77$, respectively. Most importantly, the fraction of excluded galaxies does not depend on the galaxy magnitude, and (2) it is extracted from a sample of $\sim 700,000$ SDSS galaxies over a volume of $2.5 \times 10^{5} \mathrm{Mpc}^{3}$, which encompasses the full range of local environmental densities, from voids to galaxy clusters.
} 


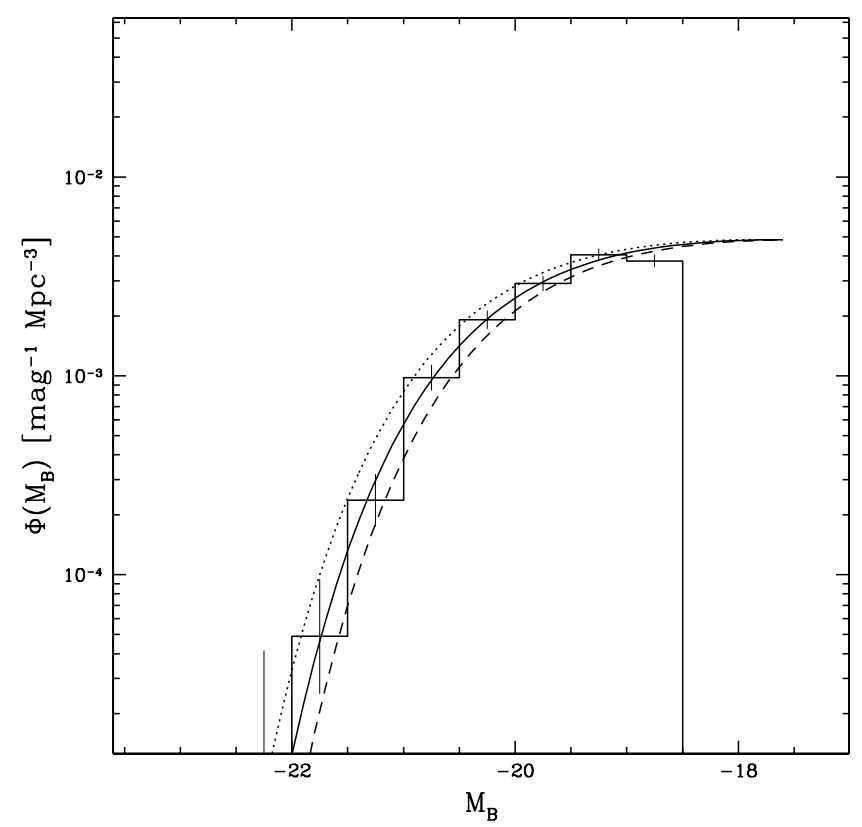

FIG. 21.- Rest-frame $B$-band LF for the SDSS galaxy sample of Kampczyk et al. (2007), which we use to quantify the evolutionary trends since $z \sim 1$ present in our COSMOS sample (solid histogram). This is normalized using the Blanton et al. (2003) $g$-band LF, converted to the $B$-band magnitude using the average transformation (solid line) between a 12 Gyr old single burst SED with a solar metallicity (dotted line) and a single-burst 0.1 Gyr old SED with a similar metallicity (dashed line).

\section{REFERENCES}

Abraham, R. G., van den Bergh, S., Glazebrook, K., Ellis, R. S., Santiago, B. X., Surma, P., \& Griffiths, R. E. 1996, ApJS, 107, 1

Abraham, R. G., van den Bergh, S., \& Nair, P. 2003, ApJ, 588, 218

Bershady, M. A., Jangren, A., \& Conselice, C. J. 2000, AJ, 119, 2645

Bertin, E., \& Arnouts, S. 1996, A\&AS, 117, 393

Blanton, M. R., et al. 2003, ApJ, 594, 186

Capak, P., et al. 2007, ApJS, 172, 99

Carollo, C. M., Danziger, I. J., \& Buson, L. 1993, MNRAS, 265, 553

Cassata, P., et al. 2005, MNRAS, 357, 903

Conselice, C. J., Bershady, M. A., \& Jangren, A. 2000, ApJ, 529, 886

Daddi, E., et al. 2005, ApJ, 631, L13

Dahari, O. 1985, ApJS, 57, 643

Davis, M., et al. 2003, Proc. SPIE, 4834, 161

de Vaucouleurs, G., de Vaucouleurs, A., Corwin, H. G., Jr., Buta, R. J., Paturel,

G., \& Fouque, P. 1991, Third Reference Catalogue of Bright Galaxies (New York: Springer)

Emsellem, E., et al. 2004, MNRAS, 352, 721

Faber, S. M., et al. 2005, preprint (astro-ph/0506044)

Feldmann, R., et al. 2006, MNRAS, 372, 565

Felten, J. E. 1976, ApJ, 207, 700

Ferreras, I., et al. 2005, ApJ, 635, 243

Frei, Z., Guhathakurta, P., Gunn, J. E., \& Tyson, J. A. 1996, AJ, 111, 174

Glasser, G. J. 1962, J. Amer. Stat. Assoc., 57, 648

Graham, A. W., \& Driver, S. P. 2005, Publ. Astron. Soc. Australia, 22, 118

Hasinger, G., et al. 2007, ApJS, 172, 29

Holden, B. P., et al. 2005, ApJ, 626, 809

Jolliffe, I. T. 1972, J. Appl. Statistics, 21, 160

Kaiser, H. F. 1960, Educ. Psychol. Measurements, 20, 141

Kampczyk, P., et al. 2007, ApJS, 172, 329

Kitzbichler, M. G., \& White, S. D. M. 2007, MNRAS, 376, 2

Koekemoer, A., et al. 2007, ApJS, 127, 196
Krist, J. E., \& Hook, R. N. 1997, HST Calibration Workshop with a New Generation of Instruments, ed. S. Castertano et al. (Baltimore: STScI), 192 Leauthaud, A., et al. 2007, ApJS, 172, 219

Lilly, S., et al. 2007, ApJS, 172, 70

Lotz, J. M., Primack, J., \& Madau, P. 2004, AJ, 128, 163

McIntosh, D. H., et al. 2005, ApJ, 632, 191

Michard, R., \& Marchal, J. 1994, A\&AS, 105, 481

Mizuno, T., \& Oikawa, K.-I. 1996, PASJ, 48, 591

Mobasher, B., et al. 2007, ApJS, 172, 117

Oke, J. B. 1974, ApJS, 27, 21

Petrosian, V. 1976, ApJ, 209, L1

Rigler, M. A., \& Lilly, S. J. 1994, ApJ, 427, L79

Sargent, M., et al. 2007, ApJS, 172, 434

Scarlata, C., et al. 2007, ApJS, 172, 406

Schade, D., et al. 1999, ApJ, 525, 31

Schmidt, M. 1968, ApJ, 151, 393

Scorza, C., \& Bender, R. 1995, A\&A, 293, 20

Scoville, N., et al. 2007a, ApJS, 172, 38

$$
\text { 2007b, ApJS, 172, } 150
$$

Simard, L. 1998, in ASP Conf. Ser. 145, Astronomical Data Analysis Software and Systems VII, ed. R. Albrecht, R. N. Hook, \& H. A. Bushouse (San Francisco: ASP), 108

Simard, L., et al. 2002, ApJS, 142, 1

Strauss, M. A., et al. 2002, AJ, 124, 1810

Taniguchi, Y., et al. 2007, ApJS, 172, 9

Thomas, D., \& Davies, R. L. 2006, MNRAS, 366, 510

Treu, T., et al. 2005, ApJ, 633, 174

Willmer, C. N. A., et al. 2006, ApJ, 647, 853

Wolf, C., Meisenheimer, K., Rix, H.-W., Borch, A., Dye, S., \& Kleinheinrich, M. 2003, A\&A, 401, 73

York, D. G., et al. 2000, AJ, 120, 1579 